\title{
X-shooter spectroscopy of young stellar objects
}

\section{Accretion in low-mass stars and substellar objects in Lupus ${ }^{\star} \star \star$}

\author{
J. M. Alcalá ${ }^{1}$, A. Natta ${ }^{2,9}$, C. F. Manara ${ }^{7}$, L. Spezzi ${ }^{7}$, B. Stelzer ${ }^{3}$, A. Frasca ${ }^{4}$, K. Biazzo ${ }^{4,1}$, E. Covino ${ }^{1}$, S. Randich ${ }^{2}$, \\ E. Rigliaco ${ }^{8}$, L. Testi ${ }^{2,7,11}$, F. Comerón ${ }^{7}$, G. Cupani $^{5}$, and V. D’Elia ${ }^{6,10}$ \\ 1 INAF - Osservatorio Astronomico di Capodimonte, via Moiariello 16, 80131 Napoli, Italy \\ e-mail: alcala@oacn.inaf.it \\ 2 INAF - Osservatorio Astrofisico di Arcetri, Largo E. Fermi 5, 50125 Firenze, Italy \\ 3 INAF - Osservatorio Astronomico di Palermo, Piazza del Parlamento 1, 90134 Palermo, Italy \\ ${ }^{4}$ INAF - Osservatorio Astrofisico di Catania, via S. Sofia 78, 95123 Catania, Italy \\ 5 INAF - Osservatorio Astronomico di Trieste, via Tiepolo 11, 34143 Trieste, Italy \\ 6 INAF - Osservatorio Astronomico di Roma, via Frascati 33, 00040 Monteporzio Catone, Italy \\ 7 European Southern Observatory, Karl-Schwarzschild-Str. 2, 85748 Garching, Germany \\ 8 Department of Planetary Science, Lunar and Planetary Lab. University of Arizona, 1629, E. University Blvd, 85719, Tucson, AZ, \\ USA \\ 9 DIAS/Dublin Institute for Advanced Studies, Burlington Road, 15 Dublin 4, Ireland \\ 10 ASI-Science Data Centre, via Galileo Galilei, 00044 Frascati, Italy \\ 11 Excellence Cluster Universe, Boltzmannstr. 2, 85748 Garching, Germany
}

Received 10 July 2013 / Accepted 3 October 2013

\section{ABSTRACT}

\begin{abstract}
We present VLT/X-shooter observations of a sample of 36 accreting low-mass stellar and substellar objects (YSOs) in the Lupus star-forming region, spanning a range in mass from $\sim 0.03$ to $\sim 1.2 M_{\odot}$, but mostly with $0.1 M_{\odot}<M_{\star}<0.5 M_{\odot}$. Our aim is twofold: firstly, to analyse the relationship between excess-continuum and line emission accretion diagnostics, and, secondly, to investigate the accretion properties in terms of the physical properties of the central object. The accretion luminosity ( $\left.L_{\text {acc }}\right)$, and in turn the accretion rate $\left(\dot{M}_{\text {acc }}\right)$, was derived by modelling the excess emission from the UV to the near-infrared as the continuum emission of a slab of hydrogen. We computed the flux and luminosity ( $L_{\text {line }}$ ) of many emission lines of $\mathrm{H}, \mathrm{He}$, and Ca II, observed simultaneously in the range from $\sim 330 \mathrm{~nm}$ to $2500 \mathrm{~nm}$. The luminosity of all the lines is well correlated with $L_{\text {acc }}$. We provide empirical relationships between $L_{\text {acc }}$ and the luminosity of 39 emission lines, which have a lower dispersion than relationships previously reported in the literature. Our measurements extend the $\mathrm{Pa} \beta$ and $\mathrm{Br} \gamma$ relationships to $L_{\text {acc }}$ values about two orders of magnitude lower than those reported in previous studies. We confirm that different methodologies of measuring $L_{\text {acc }}$ and $\dot{M}_{\text {acc }}$ yield significantly different results: $\mathrm{H} \alpha$ line profile modelling may underestimate $\dot{M}_{\text {acc }}$ by 0.6 to 0.8 dex with respect to $\dot{M}_{\text {acc }}$ derived from continuum-excess measures. These differences may explain the probably spurious bi-modal relationships between $\dot{M}_{\text {acc }}$ and other YSOs properties reported in the literature. We derived $\dot{M}_{\text {acc }}$ in the range $2 \times 10^{-12}-4 \times 10^{-8} M_{\odot} \mathrm{yr}^{-1}$ and conclude that $\dot{M}_{\text {acc }} \propto M_{\star}{ }^{1.8( \pm 0.2)}$, with a dispersion lower by a factor of about 2 than in previous studies. A number of properties indicate that the physical conditions of the accreting gas are similar over more than 5 orders of magnitude in $\dot{M}_{\text {acc }}$, confirming previous suggestions that the geometry of the accretion flow controls the rate at which the disc material accretes onto the central star.
\end{abstract}

Key words. stars: pre-main sequence - stars: low-mass - accretion, accretion disks - line: formation - line: identification open clusters and associations: individual: Lupus

\section{Introduction}

The mass accretion rate is a key quantity for studies of pre-main sequence (PMS) stellar evolution. The evolution of accretion discs around young low-mass $\left(M_{\star}<0.5 M_{\odot}\right)$ stellar and substellar objects (YSOs) is regulated by the simultaneous effects of the mass accretion onto the star and the ejection of matter from the star-disc system (Paatz \& Camenzind 1996, and references therein). Understanding the evolution of accretion discs can provide strong constraints on theories of planet formation

* Based on observations collected at the European Souther Observatory at Paranal, under programs 084.C-0269(A), 085.C0238(A), 086.C-0173(A), 087.C-0244(A) and 089.C-0143(A).

$\star \star$ Tables 1-4 and Appendices are available in electronic form at http://www. aanda.org and evolution. The mass accretion rate, $\dot{M}_{\text {acc }}$, sets an important constraint for the disc evolution models (Hartmann et al. 1998) and disc-clearing mechanisms (Alexander et al. 2006; Gorti \& Hollenbach 2009).

All young objects, from intermediate-mass stars to brown dwarfs, show evidence of circumstellar discs and accretion at some stage of their evolution. The currently accepted paradigm that explains mass accretion in YSOs is the magnetospheric accretion model (Uchida \& Shibata 1985; Königl 1991; Shu et al. 1994). The model assumes that matter from the inner edge of the accretion disc is channelled along the magnetic-field lines onto the star. The gas shocking at the stellar surface produces high temperatures $\left(\sim 10^{4} \mathrm{~K}\right)$, giving rise to continuum and line emission in the accretion flows. Balmer continuum radiation is mostly emitted in the optically thin pre-shock gas, while Paschen 
continuum emission is generally produced in optically thick post-shock gas. Emission in the Balmer lines as well as in other lines of $\mathrm{Ca}$ II and He I originates mainly in the accretion columns (Hartmann et al. 1994; Calvet \& Gullbring 1998; Muzerolle et al. 2001; Kurosawa et al. 2006). The accretion luminosity, $L_{\text {acc }}$, which measures the radiative losses, integrated over the whole spectrum, can be converted into the mass accretion rate, $\dot{M}_{\text {acc }}$, when the YSOs radius and mass are known (Gullbring et al. 1998; Hartmann 1998).

In the past, $L_{\mathrm{acc}}$ has been calculated using veiling measurements in high-resolution spectra (e.g. Hartigan et al. 1991; Hartigan \& Kenyon 2003; White \& Hillenbrand 2004, and references therein) or by modelling the Balmer and Paschen continuum excess emission with a plane-parallel slab of hydrogen in local thermodynamic equilibrium (LTE; e.g. Valenti et al. 1993; Gullbring et al. 1998; Herczeg \& Hillenbrand 2008; Rigliaco et al. 2012). More recently, the continuum excess emission has been measured by incorporating in the models multiple accretion components with a wide range of energy fluxes (Ingleby et al. 2013).

First direct measurements of UV excess emission in very low-mass stars and substellar objects were made by Herczeg \& Hillenbrand (2008), Herczeg et al. (2009), and Rigliaco et al. (2012). Prior to theses studies, the difficulty of detecting the Balmer continuum emission in brown dwarfs called for the necessity of developing magnetospheric accretion models to reproduce the $\mathrm{H} \alpha$ line profile (Muzerolle et al. 2001, 2003, 2005), which is more easily measured in these objects. These studies additionally showed that the size of the accretion flows and the mass accretion rate are correlated, suggesting that the geometry of the accretion flows controls the rate at which the disc material accretes onto the central star (Muzerolle et al. 2001).

It has been shown that $L_{\text {acc }}$, hence $\dot{M}_{\text {acc }}$, is well correlated with the line luminosity, $L_{\text {line }}$, of several emission lines of the Balmer series, the He I and Ca II lines (e.g Herczeg \& Hillenbrand 2008; Rigliaco et al. 2012, and references therein), as well as with hydrogen recombination lines in the near-infrared (Muzerolle et al. 1998; Calvet et al. 2004). This underlines the importance of these emission features as accretion diagnostics. The $\dot{M}_{\text {acc }}-L_{\text {line }}$ relationships were then extended to the substellar regime (Natta et al. 2004; Mohanty et al. 2005; Herczeg \& Hillenbrand 2008) by combining measures of $\dot{M}_{\text {acc }}$ using different methodologies for different mass regimes (spectral-veiling determination and modelling of the $\mathrm{H} \alpha$ line profile for stars with $M_{\star}$ larger and smaller than $0.3 M_{\odot}$, respectively). A two-mode relation, with respect to YSO mass, between $\dot{M}_{\text {acc }}$ and the surface flux of the Ca II infrared triplet (IRT) line at $866.2 \mathrm{~nm}$ was suggested (Mohanty et al. 2005), but the origin of the bimodality was unclear. A relationship between $\dot{M}_{\text {acc }}$ and the width of the $\mathrm{H} \alpha$ line at $10 \%$ of the line peak was also established, though with a large scatter (Natta et al. 2004).

Researchers have been using emission lines as proxies for measuring $L_{\text {acc }}$ (Muzerolle et al. 1998, 2000, 2001, 2005; Natta et al. 2004; Mohanty et al. 2005; Gatti et al. 2006, 2008; Antoniucci et al. 2011; Biazzo et al. 2012, and references therein). When applied to large samples of YSOs, the $L_{\text {acc }}-$ $L_{\text {line }}$ relations provided a correlation between accretion rate and mass of the central object, that is, $\dot{M}_{\text {acc }} \propto M_{\star}{ }^{\alpha}$, with $\alpha \approx 2$ (Muzerolle et al. 2005; Natta et al. 2006), but with a scatter of more than 2 dex in $\log \dot{M}_{\text {acc }}$ at a given YSO mass. Such a large scatter is not easily explained in terms of YSO variability (Costigan et al. 2012, 2013) or different methodological approaches used for deriving $L_{\text {acc }}$ (Herczeg \& Hillenbrand 2008).
The main caveat of the $L_{\mathrm{acc}}-L_{\mathrm{line}}$ relations is the asynchronous measuring of photometry and spectral lines, which is complicated by the fact that emission lines can trace strong winds as well as mass accretion. Additionally, emission lines are produced not only by accretion, but also in other processes such as chromospheric activity. This adds more uncertainty on the $L_{\text {acc }}$ determinations from lines. The uncertainty depends on the level of the accretion rate and on the stellar properties (see Manara et al. 2013a).

Deriving precise $L_{\text {acc }}-L_{\text {line }}$ relations for accretion requires well-calibrated observations of both the continuum and line fluxes over a wide range of wavelengths, and over the widest possible range of YSO physical parameters, that is, $L_{\text {acc }}, \dot{M}_{\text {acc }}$, and $M_{\star}$. Furthermore, an accurate knowledge of both the photospheric and chromospheric spectrum of the YSO, which need to be subtracted from the observed flux to isolate the accretion emission, is required. On the other hand, a large number of emission line diagnostics over a wide wavelength need to be studied simultaneously to avoid problems related to YSO variability to probe gas at different excitation conditions. These requirements can be fulfilled with the use of broad spectral range spectrographs such as X-shooter (Vernet et al. 2011) at the VLT. X-shooter is an ideal instrument for this purpose, as its wide wavelength range (300-2500 nm), covered in a single shot, allows comparing many different accretion diagnostics simultaneously.

In this paper we present the analysis and results of X-shooter observations of a sample of 36 accreting YSOs in the Lupus star-forming region, spanning a range in mass from $\sim 0.03$ to $1.2 M_{\odot}$, but the majority with $M_{\star}<0.5 M_{\odot}$. We use the continuum UV-excess emission as a measure of $L_{\text {acc }}$, hence of $\dot{M}_{\text {acc }}$, and provide revised relationships between $L_{\mathrm{acc}}$ and the luminosity, $L_{\text {line }}$, of an unprecedentedly large number of emission lines. The accretion properties of the sample are also analysed.

The sample and observations are presented in Sect. 2, while in Sect. 3 the sample characterisation is provided. In Sect. 4 we determine $L_{\text {acc }}$, obtained by fitting the Balmer and Paschen continua with a model of a slab of hydrogen, and derive of $\dot{M}_{\text {acc }}$ as well as the luminosity of a number of permitted emission lines. The relationships between $L_{\mathrm{acc}}$ and $L_{\text {line }}$ are derived in Sect. 5, while the accretion properties of the sample are examined in Sect. 6. The results are then discussed in Sect. 7, and our main conclusions are summarised in Sect. 8.

\section{Sample, observations, and data reduction}

As part of the consortium that built X-shooter, INAF was granted 46 nights of guaranteed-time observations (GTO) at the VLT-UT2 (Kueyen) spread over a period of three years, starting on ESO period 84. Eight of these nights were devoted to YSOs in nearby star-forming regions ( $\sim 3,3$ and 2 , nights for the $\sigma$-Ori cluster, the Lupus clouds, and the TW Hya association, respectively). The data used in this paper were acquired within the context of the X-shooter INAF/GTO (Alcalá et al. 2011) on April 06/07, 2010, April 23, 2011, and on April 18, 2012.

\subsection{Sample}

We selected YSOs in the Lupus star-forming region. The Lupus cloud complex is one of the low-mass star-forming regions located closest $(d<200 \mathrm{pc})$ to the Sun (see Comerón 2008, for a review). Similarly to other regions (e.g., Taurus, Chamaeleon, and $\rho$-Oph), a large variety of objects in various stages of evolution are present in Lupus. Several substellar objects have also 
been discovered in the region (Comerón 2008; López-Martí et al. 2003).

Our sample comprises 36 YSOs mainly in the Lupus I and III clouds that satisfy the following criteria as closely as possible: i) YSOs with infrared class II characteristics and low extinction to take full advantage of the broad X-shooter spectral range from UV to near-infrared (NIR); ii) targets extensively surveyed in as many photometric bands as possible: mainly Spitzer (IRAC and MIPS) surveys and complementary Wide-field Infrared Survey Explorer (WISE; Wright et al. 2010) data, as well as optical photometry available; and iii) mostly very low mass (VLM, $M_{\star}<$ $\left.0.3 M_{\odot}\right)$ objects, but also a number of more massive $\left(M_{\star}<\right.$ $\left.1 M_{\odot}\right)$ stars to explore a wider range of accretion luminosity.

The two main bibliographic sources from which our sample was compiled are Allen et al. (2007) and Merín et al. (2008). The former reported several new VLM YSOs with Spitzer colors, while the latter provided a clearly characterised sample in terms of spectral energy distribution (SED) and SED spectral index, based on the Spitzer c2d criteria (Evans et al. 2009). Additional class II YSOs in Lupus III that extend the sample to a broader mass range and eventually to higher accretion luminosity were selected from Hughes et al. (1994), following criteria i) and ii) above as closely as possible, although several of these targets do not possess Spitzer fluxes because they were not covered by the c2d or other Spitzer surveys. Thus, the available WISE data were used.

Among the selected objects, there are two visual binaries, namely Sz 88 and Sz123, where both components were observed by us. In another eight of our YSOs the Spitzer images revealed objects with separations between $2{ }^{\prime \prime} 0$ and $10{ }^{\prime} 0$ (see Table1), among these, six have separations larger than 4.'0 (see Table 1 in Merín et al. 2008 and Table 9 in Comerón 2008). The spatial resolution of $\mathrm{X}$-shooter is sufficiently high, allowing the observation of all our targets without light pollution from any of those nearby objects. To our knowledge, none of our targets has been reported as a spectroscopic binary in previous investigations using high-resolution spectroscopy (e.g. Melo 2003; Guenther et al. 2007). Our sample also includes Par-Lup3-4, one of the lowest-mass YSOs in Lupus known to host an outflow (Comerón et al. 2003). Although separate X-shooter papers are being devoted to studying outflows (Bacciotti et al. 2011; Whelan et al. 2013; Giannini et al. 2013), we include Par-Lup3-4 here to investigate its accretion properties in the same way as the other targets.

We stress that although the sample covers the mass range between $\sim 0.05 M_{\odot}$ and $\sim 1 M_{\odot}$ rather well (40\% with $M_{\star}<0.2 M_{\odot}, 35 \%$ with $M_{\star}$ in the range $0.2-0.5 M_{\odot}$, and $25 \%$ with $M_{\star}>0.5 M_{\odot}$ ), it is incomplete at each mass bin. However, our sample represents about $50 \%$ of the total population of class II YSOs in the Lupus I and Lupus III clouds (see Table 6 by Merín et al. 2008, for the statistics of the different YSO classes).

Table 1 provides the list of the targets. Ancillary photometric data, in the optical, near- and mid-infrared, were collected from Allen et al. (2007), Merín et al. (2008), the WISE All-Sky Source Catalog, and from an unpublished catalog by Comerón et al. (2009). The data are reported in Tables C.1 and C.2. Although not simultaneous with the X-shooter spectroscopy, these are the only photometric data available that can be used for comparisons with the spectroscopic fluxes.

\subsection{X-shooter spectroscopy}

With its three spectrograph arms, X-shooter provides simultaneous wavelength coverage from $\sim 300$ to $\sim 2480 \mathrm{~nm}$. For most of the targets, slits of $11^{\prime \prime} 0 / 0 .^{\prime} 9 / 0.9$ were used in the UVB/VIS/NIR arms, respectively, yielding resolving powers of $5100 / 8800 / 5600$. Only the two brightest objects in the sample (Sz 74 and Sz 83) were observed through the 0.5/0.4/0.4 arcsec slits in the UVB/VIS/NIR arms, respectively, yielding resolving powers of $9100 / 17400 / 10500$. Table 1 includes the observation log. Most of the targets were observed in one cycle using the A-B nodding mode, while three (Lup 706, Par-Lup3-4, and 2MASS J16085953-3856275) were observed in two cycles using the A-B-B-A nodding mode. Several class III YSOs, indistinctly quoted here as class III YSOs or class III templates, were also observed throughout the various Italian-GTO star formation runs, and their properties were published in separate papers (Manara et al. 2013a; Stelzer et al. 2013b). The spectra of these stars were used as templates for the modelling of Balmer and Paschen continua (Sect. 4.1.1) and to estimate extinction and revisit the spectral types (Sect. 3) of the class II YSOs. The class III templates cover the spectral range from K5 to M9 (see Manara et al. 2013a).

The weather conditions were mostly photometric with subarcsecond seeing except for a few minutes of thin cirrus when the seeing was $\sim 1.3$ arcsec (only for $\mathrm{Sz} 130$ ) and some clouds at the end of the night on April 06, 2010 (but only for the class III YSOs Par-Lup3-1 and Par-Lup3-2, see Manara et al. 2013a).

With a few exceptions explained below, all targets were observed at parallactic angle. The components of $\mathrm{Sz} 88$ and $\mathrm{Sz} 123$, with a separation of 1'.49 and 1.'70, respectively, were observed simultaneously by aligning the 11 aresec slit at their position angle. Likewise, for Par-Lup3-4 the slit was aligned at the position angle of the outflow (Bacciotti et al. 2011; Whelan et al. 2013).

Several telluric standard stars were observed with the same instrumental set-up and at very similar airmass as the targets. Typically, two flux standards per night were observed through a 5 arcsec slit to calibrate the flux.

\subsection{Data reduction}

The data were reduced independently for each spectrograph arm using two versions of the X-shooter pipeline (Modigliani et al. 2010), depending on the period in which the data were acquired: version 1.0.0 was used for the April 2010 data, while for the data acquired in April 2011 and April 2012, version 1.3.7 was used. The standard steps of processing included bias- or dark subtraction, flat-fielding, optimal extraction, wavelength calibration, and sky subtraction. Since version 1.0.0 did not include flux calibration, a MIDAS ${ }^{1}$ routine (see details in Alcalá et al. 2011) was used for the April 2010 data. Wavelength shifts due to instrumental flexure were corrected for using the flexcomp package within the pipeline. The precision in wavelength calibration is better than 0.01 pix in the UVB and VIS arms, corresponding to $0.002 \mathrm{~nm}$, but errors can be as large as $\sim 0.006 \mathrm{~nm}$ in the NIR arm. The flux for the April 2011 and April 2012 data was calibrated using the pipeline. A test data-set was processed with both the MIDAS procedure and the pipeline, confirming that the fluxcalibrated spectra resulting from the two procedures are identical. The final extracted one-dimensional flux-calibrated spectra, either from the MIDAS procedure or the pipeline, were not corrected for telluric absorption bands. The telluric correction was performed independently in the VIS and NIR spectra, as explained in Appendix A. The X-shooter scale of $\sim 0.16$ arcsec/pix

1 The Munich Image Data Analysis System (MIDAS) provides general tools for image processing and data reduction. It is developed and maintained by ESO. 
along the slit direction allowed us to resolve the components of the binaries, which in turn enabled us to extract the spectra of the individual components without any light contamination.

By comparing the response function of different flux standards observed during the same night, we estimate an intrinsic precision on the flux calibration of $\sim 5 \%$. This does not consider, however, errors due to flux losses induced by seeing variations during the science exposures. These errors are expected to be small (lower than 10\%), because the seeing was always subarcsecond during the science exposures. For Sz 74 and $\mathrm{Sz} 83$, both observed with the narrow slits, the seeing was $\sim 0.5$ arcsec. Therefore, $\sim 10 \%$ can be considered as a reliable relative error for the flux calibration.

The overlap of $\sim 40 \mathrm{~nm}$ between the UVB-VIS and VIS-NIR spectra allowed us to verify the consistency in flux between the different arms. While the flux match between the UVB and VIS spectra is always perfect, the NIR flux in some cases is low with respect to the VIS flux by a factor $<1.2^{2}$. Thus, an additional correction was applied to the NIR spectrum to match the flux in the VIS arm.

Finally, the ancillary photometric data were used to compare the spectroscopic fluxes with the photometric ones. The spectra follow the corresponding SED shape very well, with most of them matching the photometric fluxes within a factor of less than 1.5, whereas in some cases of objects well-known to be strongly variable (cf. Par-Lup3-4, Sz 74, Sz 83, Sz 106, Sz 113), the ratio may be as high as 2.5 . In these latter cases the difference between photometric and spectroscopic fluxes can be ascribed to variability of the object.

\section{Stellar and substellar properties}

Estimates of the physical parameters exist in the literature for some of the YSOs in our sample, but they are unknown for the majority of our targets. The quality and performance of the X-shooter spectra allow us to derive many physical quantities (cf. Manara et al. 2013b). In this paper, however, we focus only on the parameters strictly needed for studying the accretion properties of our sample. Examples of a full exploitation of the $\mathrm{X}$-shooter spectra for brown dwarfs and YSOs can be found in Alcalá et al. (2011), Stelzer et al. (2012), Manara et al. (2013a), and Stelzer et al. (2013a,b).

\subsection{Spectral type}

For the M-type stars various spectral indices were calculated following Riddick et al. (2007) for optical wavelengths, and the H2O-K2 index from Rojas-Ayala et al. (2012) for the NIR spectra. The Riddick et al. (2007) spectral indices in the VIS are almost independently of extinction ${ }^{3}$. The spectral type assigned to a given object was estimated as the average spectral type resulting from the various indices in the VIS. From the dispersion over the average of all the available spectral indices we obtain an uncertainty of half a spectral subclass.

The NIR indices provide spectral types that are consistent with the VIS results typically within one spectral subclass. Therefore, the spectral types derived from the VIS were adopted for the analysis, consistently with the spectral type assignment

\footnotetext{
2 Some flux losses in the NIR may result due to slit-vignetting caused by a slight misalignment of the NIR slit with respect to the VIS and UVB arms.

3 Note that the spectral indices may be affected by high extinction $\left(A_{V}>5 \mathrm{mag}\right)$. None of our targets has such high $A_{V}$.
}

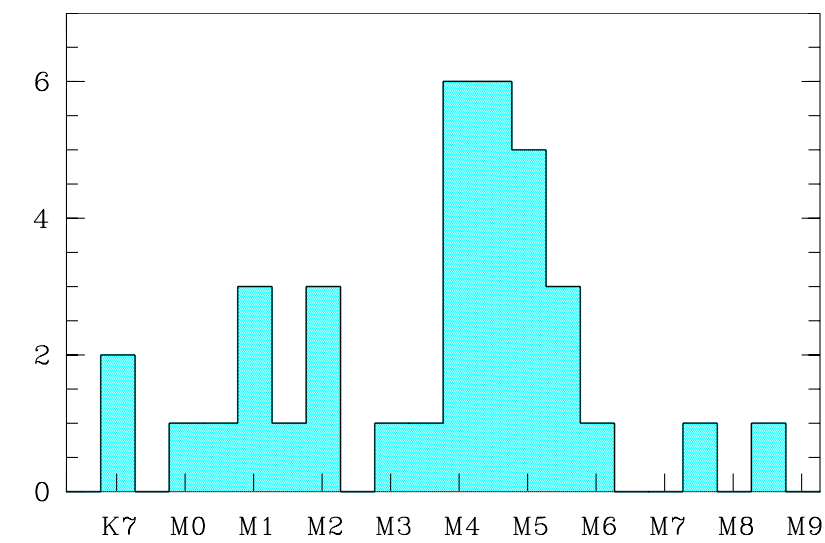

Fig. 1. Distribution of spectral types of the YSO sample.

for the class III templates (Manara et al. 2013a). The spectral types are listed in Table 2. For the two earliest-type stars in our sample (Sz73 and Sz83), an accurate spectral type of K7 is reported in the literature (see Herczeg \& Hillenbrand 2008; Comerón 2008). However, we revisit the determination using the procedures described in Sect. 3.2.

Some difference in determining the spectral type in the literature can be ascribed to the different spectral ranges used in the different investigations (Comerón et al. 2003; Hughes et al. 1994; Mortier et al. 2011). With the wide spectral range of $\mathrm{X}$-shooter we can overcome this problem. In Appendix B we investigate the impact of veiling on the spectral indices and conclude that for all M-type YSOs veiling is estimated to influence spectral type classification to less than 0.3 spectral subclasses, that is, well within the uncertainty of determining the spectral type using spectral indices. Generally, our spectral types are consistent within \pm 0.5 subclass with those in the literature, with a few exceptions that are discussed at the end of the next section. The spectral types of our sample range from K7 to M8, with an overabundance of M4-M5 objects (see Fig. 1).

\subsection{Extinction}

To derive the extinction, $A_{V}$, for a given class II YSO, its VIS spectrum was compared with the class III templates that best match the class II spectral type. All our class III templates have a low extinction $\left(A_{V}<0.5\right)$ (see Manara et al. 2013a). The templates were then artificially reddened by $A_{V}=0 . .4 .0 \mathrm{mag}$ in steps of $0.25 \mathrm{mag}$, until the best match to the class II YSO was found. To redden the spectra we used the extinction law by Weingartner \& Draine (2001), which covers a wide range in wavelength, from the UV to the mid-infrared, and has also been adopted for the c2d investigations (see Evans et al. 2009). The procedure simultaneously provided an additional test for the correct assignment of the template to derive the accretion luminosity (see Sect. 4.1.1). The $A_{V}$ values derived in this way are listed in Table 2. We confirm that the majority of the targets possess zero extinction because they were selected with this criterion. The highest values, $2.2 \mathrm{mag}$ and $3.5 \mathrm{mag}$, are found for Par-Lup3-3 and Sz73, respectively.

The main sources of uncertainty on $A_{V}$ are the errors in spectral type when associating a template to a given YSO and the error in the extinction of the template. The combined effect leads to an error of $<0.5 \mathrm{mag}$. However, another important source of uncertainty may be introduced by strong veiling, which makes the YSOs spectra intrinsically bluer than the templates. In Appendix B we investigate this effect using the object with the 
strongest veiling among the M-type YSOs in our sample, Sz 113 (see Sect. 4.1.1), and conclude that in this case we may underestimate $A_{V}$ by less than $\sim 0.5 \mathrm{mag}$. This means that the extinction as derived above is not severely affected by the veiling in our sample, but to minimize the impact of veiling, the extinction was derived only from the red portion of the spectra starting at $600 \mathrm{~nm}$. For earlier-type stars $(<\mathrm{K} 7)$ with much higher levels of veiling than those in our sample, other methods must be used to derive the extinction (see for instance Manara et al. 2013b).

To check the self-consistency of the extinction derived in another spectral range we repeated the same procedure on the NIR spectra. The result is that the $A_{V}$ values are consistent within the 0.5 mag uncertainty, but are affected by a larger error ( $\sim 0.75 \mathrm{mag})$. The latter is mainly due to the higher uncertainty in the spectral type provided by the spectral indices in the NIR than in the VIS. Therefore we adopted the values derived from the VIS for the following analysis. Another obvious reason for preferring the extinction in the VIS is that the extinction in the NIR is low and one needs to multiply it (and its uncertainty) by a large factor to derive $A_{V}$.

Our spectral type and extinction determinations agree well with the literature values except for a few cases. For Sz 69, Hughes et al. (1994) reported a spectral type M1 with a visual extinction of $3.20 \mathrm{mag}$, while in our case the M4 template with zero extinction fits the entire X-shooter spectrum much better. For Sz 110, Hughes et al. (1994) reported M2, while Mortier et al. (2011) claimed M3, more consistent with our M4 determination. In the case of Sz 113, the M4 spectral type reported by Hughes et al. (1994) agrees with our M4.5 determination, while Mortier et al. (2011) reported M1 and Comerón et al. (2003) M6. The visual extinction values in the literature for Par-Lup3-4 range from 2.4 to $5.6 \mathrm{mag}$ (Comerón et al. 2003). The confirmed subluminosity and edge-on geometry of this object (Comerón et al. 2003; Huelamo et al. 2010) suggest that our zero extinction can be interpreted as wavelength-independent, that is, gray extinction, rather than as a null extinction (see also Whelan et al. 2013). Interestingly, a null extinction is consistent with the value derived off-source using the [Fe II] lines at 1.27, 1.32, and $1.64 \mu \mathrm{m}$ (Bacciotti et al. 2011; Giannini et al. 2013), which trace jet emission (Nisini et al. 2005).

On the other hand, it is worth mentioning that the zero extinction we find for $\mathrm{Sz} 83$ agrees with the value derived by Herczeg et al. (2005) from the profile of the Ly $\alpha$ line. Our procedure also confirms the spectral type of this YSO, despite its strong veiling (see Sect. 4.1.1). Our $A_{V}$ determination for $\mathrm{Sz} 113$, the most veiled among the M-type YSOs in our sample, also agrees with that reported by Hughes et al. (1994).

\subsection{YSOs physical parameters}

The effective temperature, $T_{\text {eff }}$, was derived using the temperature scales given by Kenyon \& Hartmann (1995) for the two K-type stars, and by Luhman et al. (2003) for the M-type YSOs. The latter scale is intermediate between the dwarf and giant temperature scales, and more appropriate for young objects than temperature scales derived for field M-dwarfs (e.g. Testi 2009; Rajpurohit et al. 2013). The stellar luminosity and radius were computed using the methods described in Spezzi et al. (2008), adopting the extinction and distance values given in Table 2. The stellar radius was also determined using the flux-calibrated X-shooter spectra as explained in Alcalá et al. (2011). The good agreement between the radius calculated with the two methods (cf. Fig. 5 in Alcalá et al. 2011) also confirms the reliability of the flux calibration of the spectra. Mass and age were derived by
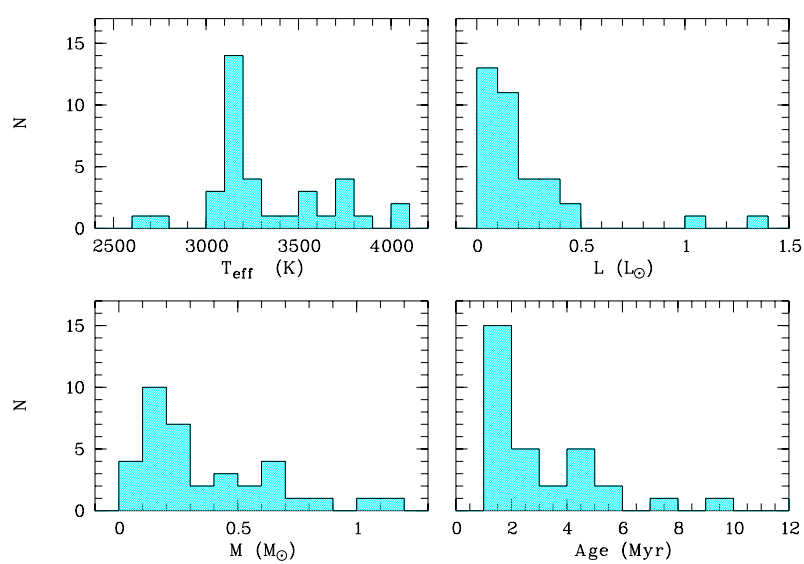

Fig. 2. Histograms of YSOs properties. The YSOs in the sample are cooler than $4000 \mathrm{~K} ; 70 \%$ are cooler than $3500 \mathrm{~K}$. The vast majority have a luminosity lower than $0.5 L_{\odot}$, while more than $70 \%$ have a mass lower than $0.5 M_{\odot}$. Two YSOs (Lup 713 and Lup 818s) have a mass just at the hydrogen-burning limit, while another two (Lup 706 and SST c2d160901.4-392512) are definitely substellar.

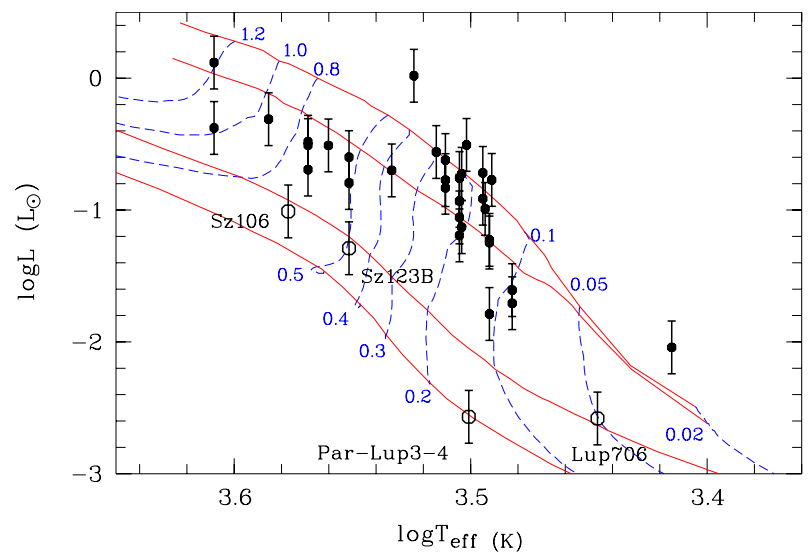

Fig. 3. Hertzsprung-Russell diagram for the Lupus sample. The four subluminous objects described in the text are represented with open circles as labelled. The continuous lines show the $1 \mathrm{Myr}, 3 \mathrm{Myr}, 30 \mathrm{Myr}$, and $10 \mathrm{Gyr}$ isochrones, reported by Baraffe et al. (1998), while the dashed lines show the low-mass PMS evolutionary tracks by the same authors as labelled.

comparison with theoretical PMS evolutionary tracks (Baraffe et al. 1998; Chabrier et al. 2000) on the HR diagram. The physical parameters of the targets are listed in Table 2 and are summarised in Fig. 2. Uncertainties in luminosity, radius, and mass take into account the error propagation of about half a spectral subclass in spectral typing, as well as errors in the photometry and uncertainty on extinction.

The luminosity of four objects, namely Par-Lup3-4, Lup706, $\mathrm{Sz} 123 \mathrm{~B}$, and $\mathrm{Sz} 106$, is significantly lower than for the other YSOs of similar spectral type or mass, hence their ages are apparently older than 15 Myr. The subluminosity of these objects compared with the others is evident in Fig. 3, where the HR-diagram for the sample is shown. It is not entirely clear whether the relatively low luminosity of these objects is due to evolution or to obscuration effects because of a particular disc geometry. Subluminosity has been reported for Sz 106 and ParLup3-4 (Comerón et al. 2003), and for the latter it has been shown that the disc is edge-on (Huelamo et al. 2010). No evidence of subluminosity for the other two objects is found in the literature. At the end of the paper (Sect. 7.4) we provide 
arguments suggesting that the subluminosity of these objects is most likely due to geometrical effects.

The average age of $3 \pm 2 \mathrm{Myr}$ for the sample, excluding the subluminous objects ${ }^{4}$, is consistent with previous age estimates for Lupus YSOs (Comerón 2008, and references therein). We did not detect statistically significant differences among the stellar parameters of the Lupus I and Lupus III YSOs because our number statistics is small and this work deals only with class II YSOs. Note, however, that a recent investigation (Rygl et al. 2013) based on Herschel data of significant samples of all classes of YSOs concluded that there are differences in the star formation rate between the various Lupus clouds.

Finally, the Li I $\lambda 670.78 \mathrm{~nm}$ absorption line is detected in all the spectra and is identified for the first time in nine of our targets, two of which are part of the class III sample (Manara et al. 2013a). Of these nine, three objects previously considered only as candidates were confirmed to be truly YSOs. We also stress that for the stars Sz 105 and Sz94, originally included in the X-shooter observations, no lithium absorption was detected, despite the high signal-to-noise ratio of their spectra. Therefore, they were not considered in our analysis. The nature of the class III source Sz 94 has been discussed in Manara et al. (2013a) and Stelzer et al. (2013b), whereas the YSO nature of $\mathrm{Sz} 105$ is unclear, but will be discussed in a future work.

\section{Accretion diagnostics}

The energy loss per unit time of the accretion energy, or accretion luminosity $L_{\text {acc }}$, is detected as continuum- and line emission over a wide spectral range. The contribution of the lines to $L_{\text {acc }}$ is generally ignored because the continuum emission is typically higher, but as we show in Sect. 4.5, significant energy losses also occur in line emission. In this section we derive the accretion luminosity of each YSO in our sample from the continuum-excess measurements, which we describe as the continuum emission of a slab of hydrogen. Previous determinations of $L_{\mathrm{acc}}$ in the literature have also excluded line emission from estimates of the accretion excess emission, making our results directly comparable with the earlier studies. The slab modelling also accounts for the excess emission shortward of $330 \mathrm{~nm}$, which is about the shortest wavelength into which our spectra are useful, and allows us to describe the excess emission in the Paschen continuum, where it is measured at very few wavelengths only, as line veiling.

\subsection{Continuum emission}

In accreting YSOs the continuum excess emission is most easily detected as Balmer continuum emission (see Valenti et al. 1993; Gullbring et al. 1998; Herczeg \& Hillenbrand 2008; Rigliaco et al. 2012, and references therein). Except for two very low mass objects (Lup 604 and Par-Lup3-3), with noisy UVB spectra, Balmer continuum emission is evident in all the YSOs of our sample (see examples in Fig. 4). The observed Balmer jump, $\mathrm{BJ}_{\mathrm{obs}}$, that is, the ratio of the flux at $360 \mathrm{~nm}$ to the flux at $400 \mathrm{~nm}$, ranges from 0.4 to 3.7 (cf. Table 3), with only three objects (Lup 604, Par-Lup3-3, and Sz 112) having $\mathrm{BJ}_{\mathrm{obs}} \leq 0.5$. This satisfies the criterion suggested by Herczeg \& Hillenbrand (2008) that any mid-M-type dwarf with an observed Balmer jump higher than 0.5 should be considered an accretor. The intrinsic Balmer jump, $\mathrm{BJ}_{\mathrm{intr}}$, which is the ratio of the flux at $360 \mathrm{~nm}$ to the flux at $400 \mathrm{~nm}$ after extinction correction and subtraction of the photospheric emission, was calculated using the

\footnotetext{
4 Note that these objects are not included in the age histogram in Fig. 2.
}
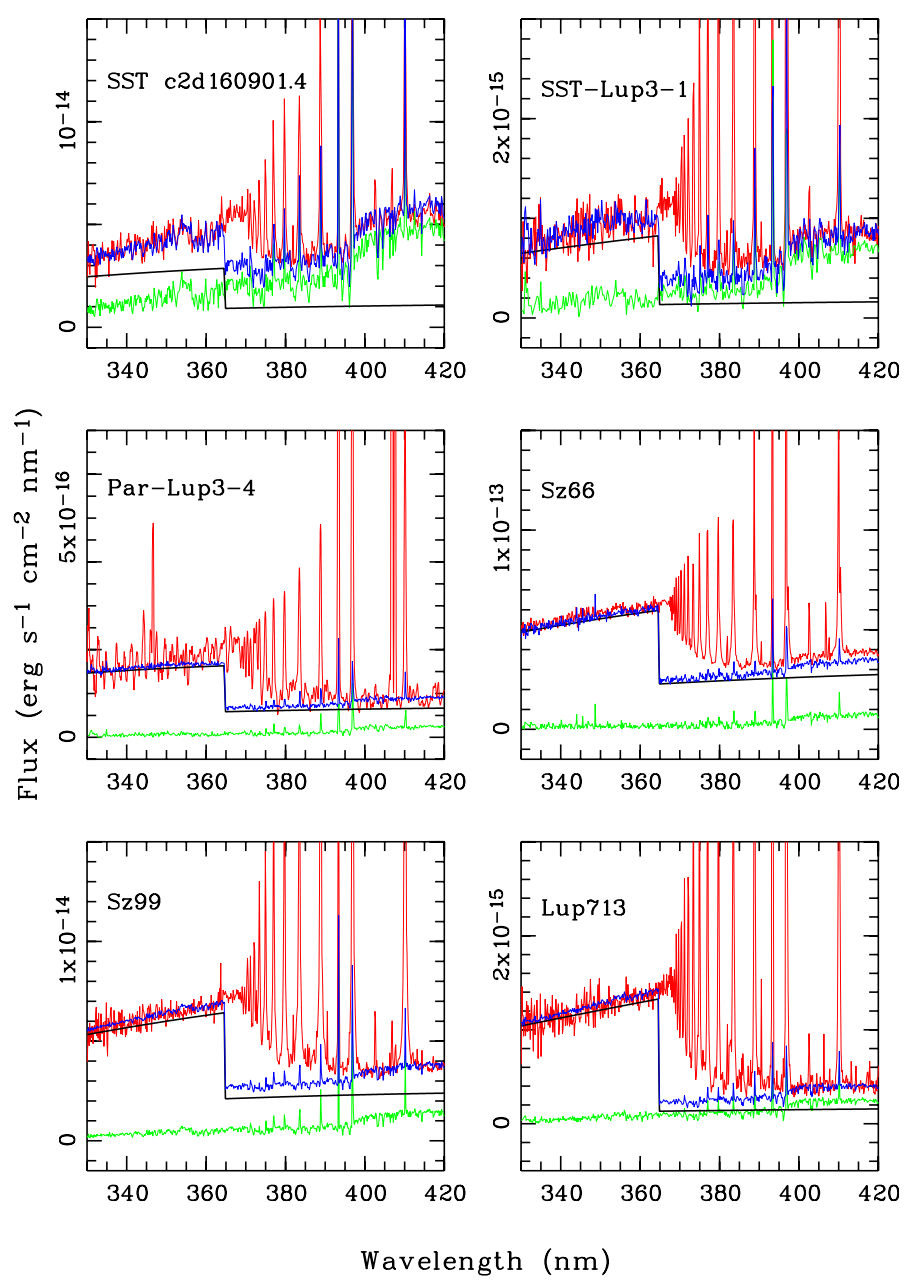

Fig. 4. Examples of X-shooter spectra of class II YSOs in the region of the Balmer jump (red lines). The spectrum of the adopted class III templates are overplotted with green lines. The continuum emission from the slab is shown by the black continuous line. The best fit with the emission predicted from the slab model added to the template is given by the blue lines.

photospheric spectrum of the same class III template as in the slab modelling described below.

Paschen continuum emission (in the wavelength range from $364 \mathrm{~nm}$ to $820 \mathrm{~nm}$ ) is dimmer than the Balmer emission in the UVB range, but is detected as veiling of the photospheric lines. Examples of this filling-in of the Ca I $\lambda 422.7 \mathrm{~nm}$ absorption line in some of the YSOs in $\sigma$-Ori are shown in Fig. 3 by Rigliaco et al. (2012; see also Fig. 2 in Herczeg \& Hillenbrand 2008). The filling-in of this line may be partially due to the Fe I emission lines at 421.6, 422.7, and $423.3 \mathrm{~nm}$. The continuum emission at wavelengths longer than about $700 \mathrm{~nm}$ is very weak in very low mass YSOs and is hardly detectable in many of our targets.

\subsubsection{Calculation of continuum accretion luminosity}

Following Valenti et al. (1993), Herczeg \& Hillenbrand (2008), and Rigliaco et al. (2012), the spectrum of each class II YSO was fitted as the sum of the photospheric spectrum and the emission of a slab of hydrogen; the accretion luminosity is given by the luminosity emitted by the slab. We took the photospheric spectrum to be that of the class III template that best matched the spectral type of the class II YSO reported in Table 2. The input spectra were extinction corrected using the extinction values reported in 
Table 2. The hydrogen slab emission includes bound-free and free-free emission from $\mathrm{H}$ and $\mathrm{H}^{-}$; it was computed assuming LTE conditions and is described by three parameters, namely the electron temperature and density and the slab length, which is related to the optical depth at $300 \mathrm{~nm}$. These three parameters determine the wavelength dependence of the excess emission. In addition, there are two "normalisation" parameters, one for the slab emission and one for the class III template flux. We varied these five parameters over a wide range of values to find the model that best matches the observed continuum of the target spectrum. More specifically, we considered a number of spectral features, namely the observed Balmer jump, the slope of the Balmer continuum measured between $~ 340$ and $360 \mathrm{~nm}$, the slope of the Paschen continuum between $\sim 400$ and $\sim 475 \mathrm{~nm}$, the value of the observed fluxes at $\sim 360 \mathrm{~nm}, \sim 460 \mathrm{~nm}$, and $\sim 710 \mathrm{~nm}$. This includes spectral regions where the emission is probably dominated by the accretion shock emission and regions where the photospheric emission is most likely the dominant component. The best-fit procedure is described in Manara et al. (2013b). After the best-fitting model was found, we determined whether it reproduced other spectral features well, in particular the $\mathrm{Ca}$ I $\lambda 422.7 \mathrm{~nm}$ absorption line. Examples of slab modelling are shown in Fig. 4, and the complete set of plots showing the fits for all targets are provided in Figs. C.1-C.4.

The adopted class III templates and the $\mathrm{BJ}_{\mathrm{obs}}, \mathrm{BJ}_{\mathrm{intr}}$, and $L_{\text {acc }}$ values corresponding to each class II YSO are reported in Table 3. In this table we also give the ratio of the excess emission over the photospheric one at $710 \mathrm{~nm}$ as in the best-fit model. The uncertainties on $L_{\mathrm{acc}}$ are dominated by the uncertainty in the extinction and by the choice of the class III template (Manara et al. $2013 \mathrm{~b}$ ). An additional, non-negligible uncertainty for low values of $L_{\text {acc }}$ comes from the uncertainty on the Paschen continuum excess emission, especially in objects with a poor signal-to-noise ratio (e.g. Rigliaco et al. 2012). In general, we estimate that the uncertainty on $L_{\text {acc }}$ is $\sim 0.2$ dex.

The Balmer and Paschen continua as well as the Balmer jump are well reproduced by our fits. On the other hand, we stress that we did not attempt to fit the hydrogen emission lines, but only the continuum emission. For this reason the region on the longer wavelength side of the Balmer jump $(\lambda \sim 365-$ $370 \mathrm{~nm}$ ) is not well reproduced by our fits, because the emission in this region originates from a superposition of unresolved hydrogen emission lines and not from continuum emission. In some cases, even when the lines are clearly resolved, the overlapping of their wings produces a pseudo-continuum that we did not account for. Such line blending shifts the apparent Balmer jump to $\sim 370 \mathrm{~nm}$, while the actual jump is at $364.6 \mathrm{~nm}$. In Sect. 4.5 we estimate the contribution of this effect on the total budget of the Balmer continuum emission.

Only in one case, namely Sz123B, the slope of the Balmer continuum is not exactly reproduced by any combination of our free parameters. We checked that this is not caused by data reduction problems (e.g. flat-field correction or incorrect spectrum extraction), but perhaps to slit-loss effects. Small differences between the observed and best-fit spectra in the Paschen continuum are present in some objects (e.g. AKC2006-19, Sz115), but differences are small compared with the excess emission in the Balmer continuum region. We were always able to fit our observations with a single slab model, without the necessity of multiple accretion components (Ingleby et al. 2013), and our fits reproduce the observed spectra well on a large wavelength interval, from $\sim 330 \mathrm{~nm}$ to $\sim 720 \mathrm{~nm}$.

In about $50 \%$ of the objects, the excess emission in the Paschen continuum accounts for more than $50 \%$ of $L_{\text {acc }}$, and in all the targets at least $30 \%$ of the total excess is emitted in that region.

\subsection{Mass accretion rate}

The accretion luminosities were converted into mass accretion rates, $\dot{M}_{\text {acc }}$, using the relation

$\dot{M}_{\mathrm{acc}}=\left(1-\frac{R_{\star}}{R_{\mathrm{in}}}\right)^{-1} \frac{L_{\mathrm{acc}} R_{\star}}{G M_{\star}} \approx 1.25 \frac{L_{\mathrm{acc}} R_{\star}}{G M_{\star}}$

where $R_{\star}$ and $M_{\star}$ are the YSO radius and mass reported in Table 2, respectively, and $R_{\text {in }}$ is the YSO inner-disc radius (Gullbring et al. 1998; Hartmann 1998). The latter corresponds to the distance from the star at which the disc is truncated due to the stellar magnetosphere - and from which the disc gas is accreted, channelled by the magnetic field lines. It has been found that $R_{\text {in }}$ ranges from $3 R_{\star}$ to $10 R_{\star}$ (Johns-Krull 2007). For consistency with previous studies (e.g. Gullbring et al. 1998; Herczeg \& Hillenbrand 2008; Rigliaco et al. 2012), here we assumed $R_{\text {in }}$ to be $5 R_{\star}$. The results on $\dot{M}_{\text {acc }}$ are listed in Col. 7 of Table 3.

We calculated $\dot{M}_{\text {acc }}$ values from $2 \times 10^{-12} M_{\odot} \mathrm{yr}^{-1}$ to $4 \times$ $10^{-8} M_{\odot} \mathrm{yr}^{-1}$. The sources of error in $\dot{M}_{\text {acc }}$ are the uncertainties on $L_{\text {acc }}$, stellar mass and radius (see Table 2). Propagating these, we estimate an average error of $\sim 0.35 \mathrm{dex}$ in $\dot{M}_{\text {acc }}$. However, additional errors on these quantities come from the uncertainty in distance, as well as from differences in evolutionary tracks. The uncertainty on the Lupus YSOs distance is estimated to be $\sim 20 \%$ (see Comerón 2008, and references therein), yielding a relative uncertainty of about $0.26 \mathrm{dex}$ in the mass accretion rate ${ }^{5}$. On the other hand, using the D'Antona \& Mazzitelli (1997) tracks, we obtain a difference in mass from $10 \%$ to $70 \%$ (with an average of $30 \%$ ) with respect to the Baraffe et al. (1998) tracks, leading to uncertainties of $0.04 \mathrm{dex}$ to $0.3 \mathrm{dex}$ in $\dot{M}_{\text {acc }}$. We estimate that the cumulative relative uncertainty in $\dot{M}_{\text {acc }}$ is about 0.5 dex.

With $\dot{M}_{\text {acc }}=3.4 \times 10^{-8} M_{\odot} \mathrm{yr}^{-1}$, the strongest accretor in our sample is Sz 83. A variety of $\dot{M}_{\text {acc }}$ estimates for this star exist in the literature that range from $10^{-7}$ to a few $10^{-8} M_{\odot} \mathrm{yr}^{-1}$ and may be as high as $10^{-6}$ (Comerón 2008). Our $\dot{M}_{\text {acc }}$ estimate agrees very well with that calculated by Herczeg \& Hillenbrand (2008) $\left(1.8 \times 10^{-8} M_{\odot} \mathrm{yr}^{-1}\right)$.

There are large discrepancies between our $\dot{M}_{\text {acc }}$ determinations and those derived by Comerón et al. (2003) for Sz 100, Sz 106, Sz 113, and Par-Lup3-4. The Comerón et al. (2003) estimates, which are based on the flux of the Ca II $\lambda 854.2 \mathrm{~nm}$ line, are higher by up to 1 dex. Although part of the discrepancies may in principle be ascribed to variable accretion, this variability must be enormous over timescales of years to explain the differences. Costigan et al. $(2012,2013)$ have observed variable accretion over years, but their results show that it is very rare to have YSOs that vary $\dot{M}_{\text {acc }}$ by large factors. Most of the variability they found occurs on rotational timescales, suggesting asymmetric and not strongly variable accretion flows.

\subsection{Emission lines}

We detected a large number of permitted and forbidden emission lines that displayed a variety of profiles. The analysis of forbidden emission lines is deferred to a forthcoming paper (Natta et al., in prep.). The emission lines studied here are listed in Table 4. The detection/non-detection of these lines depends on

Note that $\dot{M}_{\text {acc }} \propto d^{3}$, as $L_{\text {acc }} \propto d^{2}$ and $R_{\star} \propto d$. 
the instrumental sensitivity and on the accretion rate of the individual YSO. The number of detections of each line is given in Col. 5 of Table 4, labelled as the number of points used for the linear fits in Sect. 5.

The detected emission lines include several from the Balmer and Paschen series, the Br $\gamma$ line, and several helium and calcium lines. Examples of permitted emission lines in X-shooter spectra from our GTO programme have been published in previous papers (e.g. Alcalá et al. 2011; Bacciotti et al. 2011; Rigliaco et al. 2011c, 2012; Manara et al. 2013a; Stelzer et al. 2013a).

Balmer lines are detected up to H25 in six objects (Lup 713, $\mathrm{Sz} 113, \mathrm{Sz} 69, \mathrm{Sz} 72, \mathrm{Sz} 83, \mathrm{Sz} 88 \mathrm{~A})$. One of these (Lup 713) is at the hydrogen-burning limit, with its spectrum resembling that of the young brown dwarf J 053825.4-024241 reported in Rigliaco et al. (2011b). In Sz 88A Balmer line emission is detected up to $\mathrm{H} 27$ at the $2 \sigma$ level. The $\mathrm{Pa} 8, \mathrm{~Pa} 9$, and $\mathrm{Pa} 10$ lines are located in spectral regions of dense telluric absorption bands. Although the telluric correction was performed as accurately as possible, some residuals from the correction still remain. Thus, the detection and analysis of these three Paschen lines is more uncertain and lead to larger errors, in particular for $\mathrm{Pa} 8$.

We considered the nine He I lines with the highest transition strength. Of these, the He I $\lambda 1082.9 \mathrm{~nm}$ has been found to be also related to winds and outflows (Edwards et al. 2006). Thus, the line may include both accretion and wind contributions. In most cases the He I $\lambda 492.2 \mathrm{~nm}$ is blended with the Fe I $\lambda 492.1 \mathrm{~nm}$ line, and we did not attempt any de-blending.

The Ca II H \& $\mathrm{K}$ lines are detected in all YSOs. The Ca II $\mathrm{H}$-line is partially blended with $\mathrm{H} \epsilon$. The CaII IRT $\lambda \lambda \lambda 849.8$, $854.2,866.2 \mathrm{~nm}$, and the D-lines of the Na I $\lambda \lambda 589.0,589.6 \mathrm{~nm}$ doublet are very well resolved in all our spectra. In several objects both the Ca II IRT and the Na I lines are detected as an emission reversal superposed on the broad photospheric absorption lines. Therefore we corrected the strength of these lines for the photospheric contribution. This was done for the complete sample (see Sect. 4.4).

Finally, the two O I lines at $777.3 \mathrm{~nm}$ and $844.6 \mathrm{~nm}$ are clearly detected in 14 and 18 YSOs, respectively. These lines are seen in the objects with the strongest Balmer, He I, and Ca II lines.

\subsection{Line fluxes and equivalent widths}

The flux in permitted lines was computed by directly integrating the flux-calibrated and extinction-corrected spectra using the splot package under IRAF ${ }^{6}$. Three independent measurements per line were made, considering the lowest, highest, and the middle position of the local continuum, depending on the local noise level of the spectra. The flux and its error were then computed as the average and standard deviation of the three independent measurements, respectively. The extinction-corrected fluxes, equivalent widths, and their errors are reported in several tables provided in Tables C. 3 to C. $11^{7}$. In the cases where the lines were not detected, $3 \sigma$ upper limits were estimated using the relationship $3 \times F_{\text {noise }} \times \Delta \lambda$, where $F_{\text {noise }}$ is the rms flux-noise in

\footnotetext{
6 IRAF is distributed by the National Optical Astronomy Observatory, which is operated by the Association of the Universities for Research in Astronomy, inc. (AURA) under cooperative agreement with the National Science Foundation.

7 The flux errors reported in these tables are those resulting from the uncertainty in continuum placement. The estimated $\sim 10 \%$ uncertainty of flux calibration (see Sect. 2.3) should be added in quadrature.
}

the region of the line and $\Delta \lambda$ is the expected average line width, assumed to be $0.2 \mathrm{~nm}$.

For the $\mathrm{H} \epsilon$ line, blended with the $\mathrm{Ca}$ II $\mathrm{H}$ line, measurements of both lines were attempted by a de-blending procedure using IRAF. In many cases, the X-shooter resolution allowed us to resolve both lines almost entirely. However, 11 YSOs display very broad lines, making the de-blending measurements unreliable. These objects are flagged in Tables C. 3 and C. 10 .

The contribution of the photospheric absorption lines of the $\mathrm{Na}$ I D lines and the Ca II IRT, strongest in the late-K and earlyto-mid M-type objects, were removed in all spectra by using the synthetic BT-Settl spectra by Allard et al. (2012) of the same $T_{\text {eff }}$ and $\log g$ as the YSOs, binned at the same resolution of X-shooter, and were rotationally broadened at the same $v \sin i$ as the YSOs. For this purpose, we applied the ROTFIT code (Frasca et al. 2006), specifically modified for X-shooter data (See Stelzer et al. 2013b, for details). In some objects with very broad emission lines, the Ca II IRT lines are fully blended with the Paschen lines Pa 13 ( $\lambda 866.502$ nm), Pa 15 $(\lambda 854.538 \mathrm{~nm})$, and $\mathrm{Pa} 16(\lambda 849.249 \mathrm{~nm})$. These objects are flagged in Table C.10.

We note that the ratio of the Ca II IR triplet lines is always very close to $1: 1: 1$ (see Table C.10), that is, consistent with optically thick gas conditions (Herbig \& Soderblom 1980; Hamann \& Persson 1992, and references therein), suggesting that the lines are formed in a high-density region near the surface of the central YSO, and not in a low-density outflow environment (see Reipurth et al. 1986; Graham \& Heyer 1988; Fernández \& Comerón 2001).

Although several objects display hydrogen recombination lines with high quantum numbers, we restricted our analysis to Balmer lines up to H15, Paschen lines up to Pa 10, and the $\mathrm{Br} \gamma$ line, as well as the helium, calcium, sodium, and oxygen lines listed in Table 4.

The luminosity of the different emission lines was computed as $L_{\text {line }}=4 \pi d^{2} f_{\text {line }}$, where $d$ is the YSO distance in Table 2 and $f_{\text {line }}$ is the extinction-corrected absolute flux of the lines reported in Tables C. 3 to C. 11 .

\subsection{Lines versus continuum losses}

We calculated the total line luminosity, $L_{\text {all_lines }}$, as the sum of the luminosity of all the emission lines detected in every YSO. In the integrated luminosity of the Balmer lines we also accounted for the flux of the pseudo-continuum produced by line blending close to the Balmer jump. This was measured by subtracting the flux of the best-fit model from the extinction-corrected spectrum of the YSO. On average, more than $70 \%$ of the total line luminosity comes from the Balmer lines. In most cases (90\%), the integrated Balmer line luminosity amounts to more than $60 \%$ of $L_{\text {all_lines }}$, while for a few objects with very strong lines (e.g. $\mathrm{Sz} 72, \mathrm{Sz} 83, \mathrm{Sz} 88 \mathrm{~A}, \mathrm{Sz} 113$ ) the emission in other lines may be as high as $55 \%$ of $L_{\text {all_lines. }}$.

The integrated line luminosity is strongly correlated with $L_{\text {acc }}$ (Fig. 5). A linear fit yields $\log \left(L_{\text {all_lines }} / L_{\odot}\right)=0.86 \times$ $\log \left(L_{\text {acc }} / L_{\odot}\right)-1.05$, with a standard deviation $\sigma=0.25$. This can be expressed as $\log \left(L_{\text {all_lines }} / L_{\text {acc }}\right)=-0.14 \log \left(L_{\text {acc }} / L_{\odot}\right)-1.05$. From the last equation and considering the scatter of the relation, we calculated that objects with $L_{\text {acc }}<10^{-4} L_{\odot}$ (only five YSOs) may have $L_{\text {all_lines }} / L_{\text {acc }}$ ratios as high as 0.55 , while the ratio is lower for high $L_{\mathrm{acc}}$ and is $<0.25$ for $L_{\mathrm{acc}}>10^{-3} L_{\odot}$. Column 8 of Table 3 lists the $L_{\text {all_lines }} / L_{\text {acc }}$ ratios. Although these numbers show that $L_{\text {all_lines }}$ is a fraction of $L_{\text {acc }}$, there is considerable emission also in the lines. 


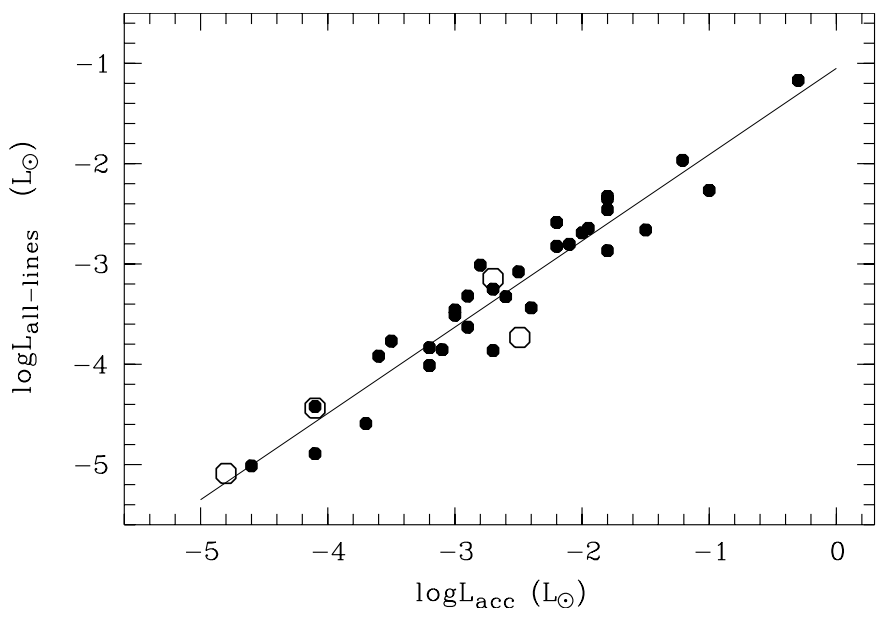

Fig. 5. Total emission line luminosity as a function of $L_{\mathrm{acc}}$. The $L_{\mathrm{acc}}$ values were derived from the slab model in Sect. 4.1.1. Symbols are the same as in Fig. 3. The continuous line represents the linear fit discussed in the text.

\section{Relationships between continuum excess luminosity and emission line luminosity}

In units of $L_{\odot}$, the dynamical range of $L_{\text {acc }}$ for our sample covers more than four orders of magnitude, while the luminosity of the line diagnostics discussed in the previous section spans more than five orders of magnitude. This allowed us to investigate the relationships between continuum excess emission and the emission in individual permitted lines.

\subsection{Continuum vs. line emission relationships}

Figures C. 5 to C. 10 show the relationships between $L_{\text {acc }}$ and the luminosity of all the permitted emission lines discussed in Sect. 4.3. When available, values of $L_{\mathrm{acc}}$ and $L_{\text {line }}$ from previous investigations of YSOs in Taurus (cf. Herczeg \& Hillenbrand 2008, and references therein) and the $\sigma$-Ori cluster (Rigliaco et al. 2012) are overlaid.

Linear fits of the $\log L_{\text {acc }}$ vs. $\log L_{\text {line }}$ relationships were calculated using the package ASURV (Feigelson \& Nelson 1985) under the IRAF environment. ASURV includes censoring of upper or lower limits in the fits. In our various relationships the upper limits in the independent variable $L_{\text {line }}$ are well consistent with the trends seen in the $L_{\text {acc }}$ vs. $L_{\text {line }}$ plots. The results of the fits (cf. Table 4) including and excluding upper limits are consistent within the errors. The total number of points and the standard deviation of the fits are given in the fifth and sixth columns of Table 4, respectively. The errors in the computed relationships also account for upper limits when included. No fits were calculated for the $\operatorname{Br} 8(\mathrm{Br} \delta)$ relation, because the number of upper limits is larger than the number of detections, and the relationship is very scattered (see Fig. C.7).

The trends in our $L_{\text {acc }}$ vs. $L_{\text {line }}$ relationships generally agree with those found in previous investigations (Muzerolle et al. 1998; Calvet et al. 2004; Natta et al. 2004; Herczeg \& Hillenbrand 2008; Ingleby et al. 2013) (see Figs. C.5 to C.10). However, because of the different methodologies adopted in deriving $L_{\mathrm{acc}}$ ( $\mathrm{H} \alpha$ line profile modelling, veiling in the FUV, $\mathrm{UV}$, and VIS, etc.), systematic differences may exist at different mass regimes (see Herczeg \& Hillenbrand 2008, and next section). Therefore, except for the YSOs in $\sigma$-Ori (Rigliaco et al. 2012), whose $L_{\text {acc }}$ and $L_{\text {line }}$ values were computed in the same way as here, we did not combine other literature data to derive $L_{\text {acc }}-L_{\text {line }}$ relationships. Note also that our sample comprises $L_{\text {acc }} \leq 1 L_{\odot}$, while literature data extend to higher accretion luminosities.

While the accretion luminosity is well correlated with the luminosity of all the emission lines, the scatter in the correlations differs for the various lines (see standard deviation from the fits in Table 4).

\subsection{Comparison with previous relationships}

Figures C.5 to C. 10 show that the $L_{\text {acc }}-L_{\text {line }}$ relations for the Lupus YSOs are fairly consistent with those in previous investigations of continuum excess in YSOs in Taurus (cf. Herczeg \& Hillenbrand 2008, and references therein), and $\sigma$-Ori (Rigliaco et al. 2012). The slopes and zero points of the $L_{\text {acc }}$ Vs. $L_{\text {line }}$ relations derived here are consistent within the errors with those reported in Herczeg \& Hillenbrand (2008; see their Table $16^{8}$ ). A comparison of the $L_{\text {acc }}$ values derived here from the slab model with the average accretion luminosity drawn from different line diagnostics (e.g. $\mathrm{H} \alpha, \mathrm{H} \beta, \mathrm{H} \gamma, \mathrm{H} \delta$, the $\mathrm{He}$ I lines at $501.6 \mathrm{~nm}$ and $587.6 \mathrm{~nm}$, the He II line at $468.5 \mathrm{~nm}$ and the $\mathrm{Ca}$ II $\mathrm{K}$ line) as measured from the X-shooter spectra (fluxes in Tables C.3-C.11) and using the Herczeg \& Hillenbrand (2008) relationships leads to a rms difference $<0.3$ dex. This is significantly lower than the error drawn from the application of single-diagnostic relationships (see also Rigliaco et al. 2012). We also note that our relationships for the $\mathrm{H} \alpha, \mathrm{H} \beta$, and $\mathrm{Ca}$ II $\mathrm{K}$ lines are practically identical to those of Ingleby et al. (2013), which were derived by fitting $\mathrm{UV}$ and optical spectra with multiple accretion components.

Our $L_{\mathrm{acc}}-L_{\mathrm{Pa} \beta}$ and $L_{\mathrm{acc}}-L_{\mathrm{Br} \gamma}$ relations are also similar to those in previous works by Muzerolle et al. (1998), Natta et al. (2004), Calvet et al. (2000), and Calvet et al. (2004), but extend to much lower values of $L_{\text {acc }}$, toward the very low-mass regime (see Fig. C.7). As mentioned earlier, however, systematic differences may arise due to the different methodologies employed in deriving $L_{\text {acc }}$. For instance, the difference in $\log L_{\text {acc }}$ when using our Br $\gamma$ relationship and those of Muzerolle et al. (1998) may be up to $\sim 0.5$ dex for a typical $0.5 M_{\odot}$ T Tauri star with $L_{\mathrm{Br} \gamma} \sim$ $10^{-3} L_{\odot}$.

\subsection{Impact of chromospheric emission on $L_{\text {acc }}$ estimates}

Previous studies (Ingleby et al. 2011; Rigliaco et al. 2012; Manara et al. 2013a) have stressed the impact of chromospheric emission on $L_{\mathrm{acc}}$ at low levels of accretion. Our estimates of $L_{\mathrm{acc}}$ are not influenced by chromospheric line emission, because they are derived from the continuum excess emission. The typical continuum emission of chromospheric origin, if present, is automatically corrected for by using as templates the class III stars, instead of field dwarfs.

To investigate the possible effects of chromospheric line emission in our sample, we derived the accretion luminosity, $L_{\text {acc }}$ (lines) using emission line diagnostics and the $L_{\text {acc }}-L_{\text {line }}$ relations in Table 4 . We calculated $\left\langle\log L_{\text {acc }}\right.$ (lines) $\rangle$ as the average over fifteen diagnostics discussed in Manara et al. (2013a). In Fig. 6 the $\left\langle\log L_{\text {acc }}\right.$ (lines) $\rangle$ values and the $\left\langle\log L_{\text {acc }}\right.$ (lines) $\left./ L_{\star}\right\rangle$ ratio are plotted as a function of $T_{\text {eff }}$. The dashed lines in the figure show the level of chromospheric noise as determined by Manara et al. (2013a). The lines represent the locus below which the contribution of chromospheric emission starts to be important in

8 Note that the slope and zero points in the Herczeg \& Hillenbrand (2008) relationships are swapped in their Table 16. 


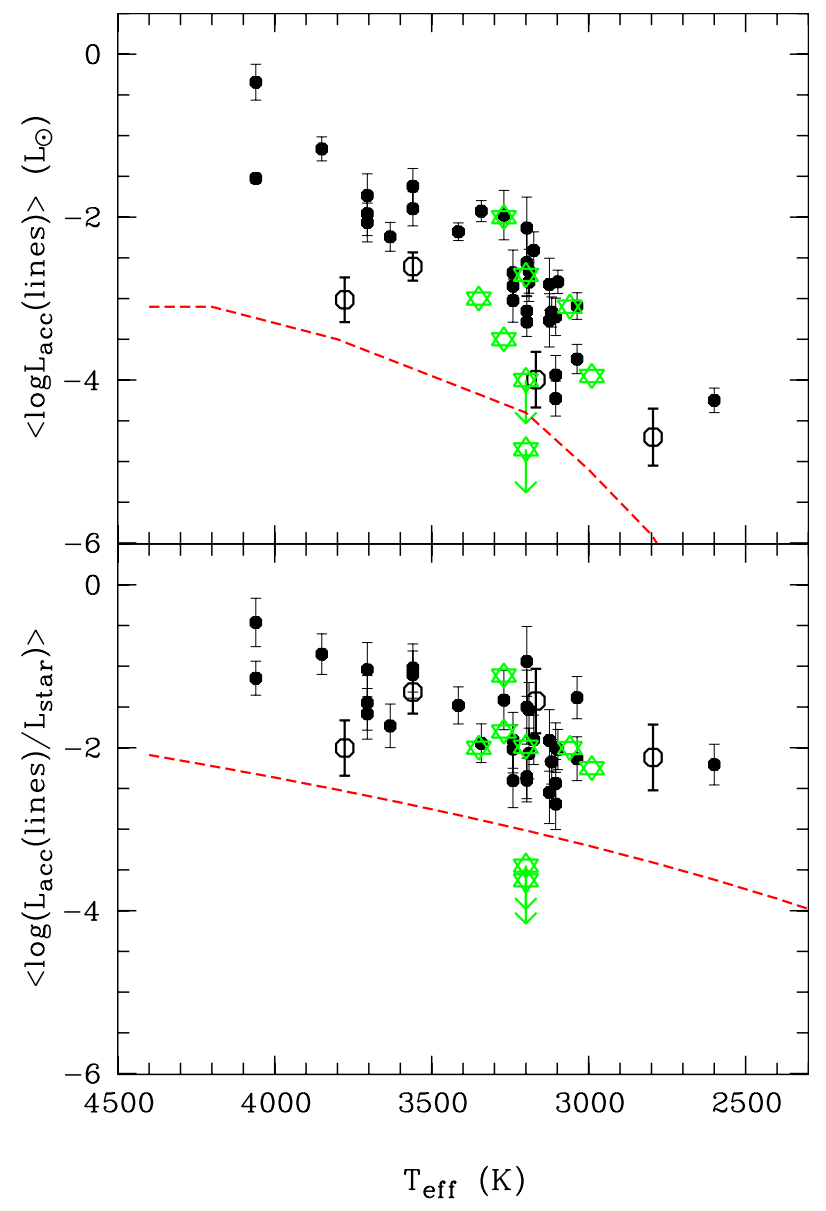

Fig. 6. Average accretion luminosity $\left\langle\log L_{\text {acc }}\right.$ (lines) $\rangle$ derived from 15 emission lines as described in the text (upper panel) and the $\left\langle\log L_{\text {acc }}\right.$ (lines) $\left./ L_{\star}\right\rangle$ ratio (lower panel) as a function of effective temperature for Lupus and $\sigma$-Ori (open stars) YSOs. Plotting symbols for the Lupus YSOs are the same as in Fig. 3. The dashed lines in both panels mark the locus below which chromospheric emission is important in comparison with $L_{\text {acc }}$. The vertical error bars represent the standard deviation over the average of 15 emission line diagnostics.

comparison with energy losses due to accretion. The accretion level of all the Lupus YSOs studied here is well above the chromospheric noise. Therefore, we conclude that the chromospheric contribution to $L_{\text {acc }}$ is influential, even at the lowest values of $L_{\text {acc }}$. Our $L_{\text {acc }}-L_{\text {line }}$ relationships are hence calculated for $L_{\text {acc }}$ values well above the chromospheric threshold. The two objects of $\sigma$-Ori indicated with upper limits correspond to SO 587 and SO 1266. For these two objects the fraction of luminosity in the Balmer lines with respect to the upper limit in $L_{\text {acc }}$ is higher than one. Rigliaco et al. (2011a, 2012) showed that in SO 587 the strong permitted lines probably originate in a photo-evaporation wind, while in SO 1266 they are dominated by chromospheric emission. We did not consider these two objects in the following plots and analyses.

\section{Accretion properties}

\subsection{Accretion luminosity versus YSO luminosity}

Previous investigations (e.g. Natta et al. 2006; Rigliaco et al. 2011a, and references therein) have shown that $L_{\text {acc }}$ and stellar luminosity in class II YSOs are correlated, although

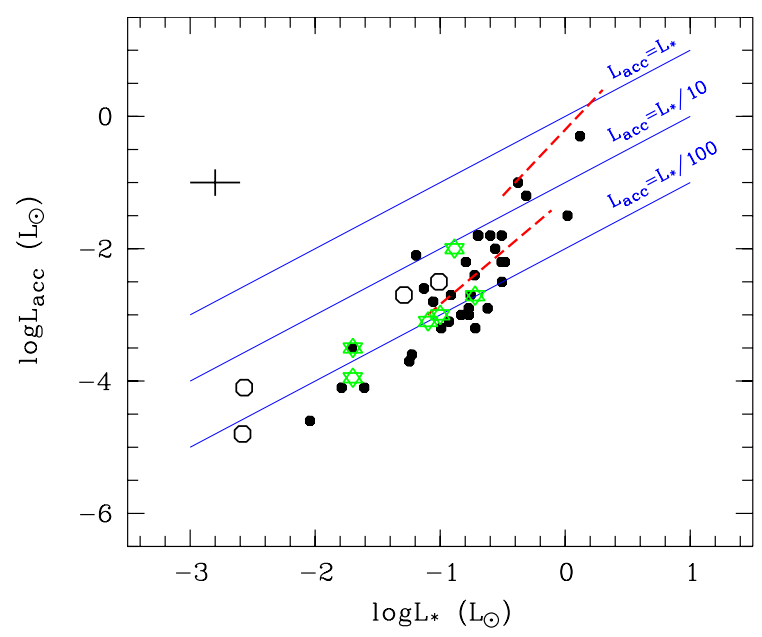

Fig. 7. Accretion luminosity as a function of stellar luminosity for Lupus and $\sigma$-Ori YSOs. Symbols are the same as in Fig. 6. The two Lupus YSOs with the lowest luminosities are Par-Lup3-4 and Lup 706. The continuous lines represent the three $L_{\text {acc }}$ vs. $L_{\star}$ relations as labelled. Average error bars are shown in the upper left. The red dashed lines are the model tracks by Tilling et al. (2008) as follows: the upper line plots $1.0 M_{\odot}$ YSOs and $f_{\text {disc }}=0.2$, while the lower one delineates $0.4 M_{\odot}$ YSOs with $f_{\text {disc }}=0.014$.

with significant scatter at a given YSO luminosity. Clarke \& Pringle (2006) pointed out that the distribution of points in the $L_{\text {acc }}-L_{\star}$ plane more or less fills a region that is bounded by the $L_{\text {acc }}=L_{\star}$ relation at high $L_{\text {acc }}$, but which is dominated by detection biases at low values of $L_{\text {acc }}$, roughly following a powerlaw $L_{\text {acc }} \propto L_{\star}{ }_{\star}^{1.6}$. This agrees with the relationships they derived from the data (detections and upper limits) in Natta et al. (2006). Tilling et al. (2008) presented simplified stellar evolution calculations for stars subject to time-dependent accretion history, and derived evolutionary tracks on the $L_{\mathrm{acc}}-L_{\star}$ diagram for a variety of fractional disc masses, $f_{\text {disc }} \equiv M_{\text {disc }} / M_{\star}$, and YSO masses. Using the D'Antona \& Mazzitelli (1997) models, they assumed that the mass accretion rate declines with time as $t^{-\eta}$, with $\eta=1.5$.

As shown in Fig. 7, the Lupus YSOs also fall below the $L_{\text {acc }}=L_{\star}$ boundary, with a small number of objects between 0.1 and $1 L_{\odot}$, and many with $L_{\text {acc }} / L_{\star}<0.01$. The data points are apparently less scattered than those of previous samples. Our sample lacks YSOs with $L_{\text {acc }}>1 L_{\odot}$, which is most likely the reason why we do not populate the region between $L_{\mathrm{acc}}=0.1 L_{\star}$ and $L_{\text {acc }}=L_{\star}$ on the diagram. The slope for the data in Fig. 7 is steeper than the $L_{\mathrm{acc}} / L_{\star}=$ constant lines, more or less following the slope of the Tilling et al. (2008) tracks. A linear fit to the data yields $L_{\text {acc }} \propto L_{\star}{ }^{1.7}$, which is very similar to the claim by Clarke \& Pringle (2006) for the lower envelope of the $L_{\mathrm{acc}}-$ $L_{\star}$ relation, but based on detections only. Note that Manara et al. (2012) found an almost identical power-law $\left(L_{\mathrm{acc}} \propto L_{\star}{ }^{1.68}\right)$ using a complete sample in the Orion Nebula Cluster, but with $L_{\text {acc }}$ determinations based on deep photometry.

According to the Tilling et al. (2008) model and a qualitative comparison between their evolutionary tracks and our data set on the $L_{\text {acc }}-L_{\star}$ diagram (Fig. 7), one would expect the discs of the lowest mass YSOs to have masses lower than $0.014 M_{\star}$. However, within the uncertainties of the measurements reported by Ricci et al. (2010), there is no evidence of a scaling between the disc mass and the stellar mass, or the mass accretion rate. Note also that a recent compilation for a wide range of masses 


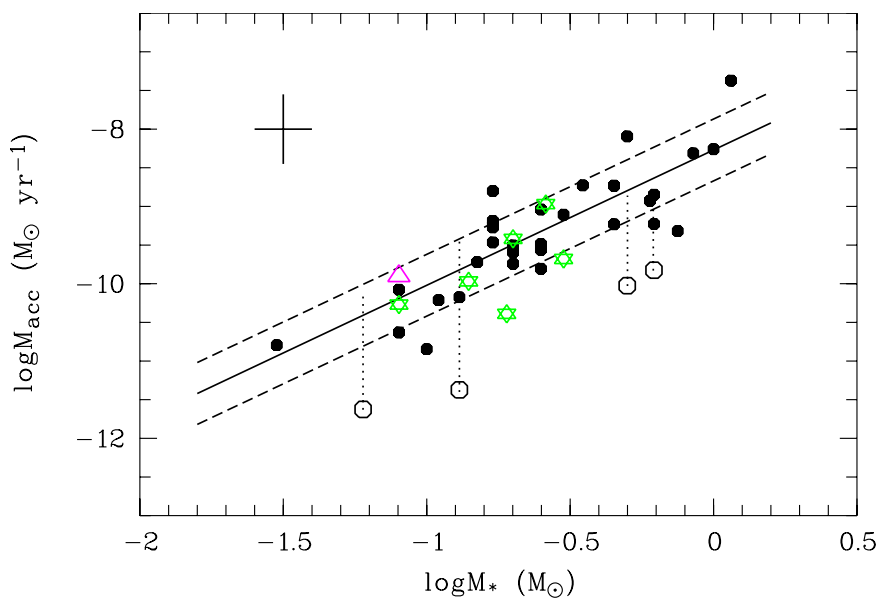

Fig. 8. Mass accretion rate $\dot{M}_{\text {acc }}$ as a function of mass. Symbols are as in Fig. 6. The vertical shifts in $\dot{M}_{\text {acc }}$ for the low-luminosity YSOs (open circles), after correcting for obscuration as explained in Sect. 7.4, are shown by the dotted lines. The magenta triangle represents the young brown dwarf FU Tau A, whose $\dot{M}_{\text {acc }}$ and $M_{\star}$ values were derived by Stelzer et al. (2013a). The continuous line represents the linear fit of Eq. (3), that is, it does not include the subluminous objects. The dashed lines represent the $1 \sigma$ deviation from the fit. Average error bars are shown in the upper left corner.

suggests that the fractional disc mass is compatible with an uniform distribution around the value $f_{\text {disc }} \approx 0.01$ (Olofsson et al. 2013).

\subsection{Accretion rate versus mass}

Previous investigations (Muzerolle et al. 2003; Mohanty et al. 2005; Natta et al. 2006; Herczeg \& Hillenbrand 2008; Rigliaco et al. 2011a; Antoniucci et al. 2011; Biazzo et al. 2012, and references therein) have found that $\dot{M}_{\text {acc }}$ goes roughly as the square of $M_{\star}$ although with a significant scatter (up to 3 dex) in $\dot{M}_{\text {acc }}$ for a given YSO mass.

Figure 8 shows the $\dot{M}_{\text {acc }}$ versus $M_{\star}$ diagram for the Lupus YSOs studied here. The position of the $\sigma$-Ori stars in this plot is consistent with Lupus and the young brown dwarf FU Tau A, also investigated with X-shooter (Stelzer et al. 2013a), follows the trend as well. The scatter is significantly increased by the four subluminous YSOs. A linear fit to the complete Lupus sample yields

$\log \dot{M}_{\mathrm{acc}}=1.89( \pm 0.26) \log M_{\star}-8.35( \pm 0.18)$

with a standard deviation of 0.6. The scatter decreases if the subluminous objects are excluded from the fit, yielding

$\log \dot{M}_{\mathrm{acc}}=1.81( \pm 0.20) \log M_{\star}-8.25( \pm 0.14)$

with a standard deviation of 0.4 . The slope and zero point of the $\dot{M}_{\text {acc }}-M_{\star}$ fit do not change significantly in either cases because the subluminous objects represent only $11 \%$ of our sample. It is thus reasonable to conclude that for our sample $\dot{M}_{\text {acc }} \propto M_{\star}{ }^{1.8( \pm 0.2)}$, which agrees with previous studies (Natta et al. 2006; Muzerolle et al. 2005; Herczeg \& Hillenbrand 2008; Rigliaco et al. 2011a; Antoniucci et al. 2011; Biazzo et al. 2012; Manara et al. 2012), but is inconsistent with the results by Fang et al. (2009) $\left(\dot{M}_{\text {acc }} \propto M_{\star}^{3}\right)$ for their subsolar mass sample in the Lynds $1630 \mathrm{~N}$ and 1641 clouds in Orion. This inconsistency is more likely to be related to the different methodologies used in deriving both $\dot{M}_{\text {acc }}$ and $M_{\star}$ than to different environmental conditions.

\section{Discussion}

\subsection{Emission lines as tracers of accretion}

Fitting the UV excess emission, and in general continuum excess emission, is the most reliable and accurate method to derive accretion luminosity in low-extinction YSOs (Muzerolle et al. 2003; Herczeg \& Hillenbrand 2008; Rigliaco et al. 2012; Ingleby et al. 2013). In the absence of UV spectra, $L_{\text {acc }}$, hence $\dot{M}_{\text {acc }}$, can be calculated using emission line diagnostics. While the relationships by Herczeg \& Hillenbrand (2008) are based on simultaneous low-resolution UV and optical spectroscopy, ours encompass and extend simultaneous observations of the diagnostics from $\sim 330 \mathrm{~nm}$ up to $\sim 2500 \mathrm{~nm}$, at intermediate spectral resolution.

Because they are based on almost twice the number of points as in previous works, the $L_{\text {acc }}-L_{\text {line }}$ relations computed here have in general a lower dispersion than those found in the literature by applying similar methodologies (e.g. Herczeg \& Hillenbrand 2008; Rigliaco et al. 2012; Ingleby et al. 2013). As in these works, each point in the relationships represents an instantaneous snapshot of $L_{\mathrm{acc}}$ and $L_{\text {line. }}$. However, results of temporal monitoring of several YSOs indicate variability in optically thick line fluxes, without significant changes in the corresponding continuum accretion rate (e.g. Gahm et al. 2008; Herczeg et al. 2009), so that some dispersion may still arise from variability even when the observations are simultaneous. Long-term spectrophotometric monitoring of YSOs over a range of masses is still required to shed light on the magnitude of this effect.

The strongest line in optical spectra of YSOs is the $\mathrm{H} \alpha$ line. Nevertheless, similarly as for the Taurus sample (Herczeg \& Hillenbrand 2008), the $L_{\mathrm{acc}}-L_{\mathrm{H} \alpha}$ relation for the Lupus YSOs is the most scattered among the Balmer lines relationships (see Table 4). This is expected because it is well known that several other processes (e.g. outflows, hot spots, chromospheric activity, complex magnetic field topology and geometry, stellar rotation) in addition to accretion may contribute to the strength of the line. All these processes have an important impact on the line profile, in particular on its width. Previous investigations have used the full width of the $\mathrm{H} \alpha$ line at $10 \%$ of the line peak, $\mathrm{WH} \alpha(10 \%)$ expressed in $\mathrm{km} \mathrm{s}^{-1}$, to investigate accretion (see White \& Basri 2003; Natta et al. 2004, and references therein). Since $\mathrm{WH} \alpha(10 \%)$ is easily gathered from optical spectra, many authors have used it to estimate $\dot{M}_{\text {acc }}$. Nevertheless, as discussed by many authors, the relationship has a very large scatter and its use is discouraged when reliable measurements of the line luminosity are possible. In Natta et al. (2004) the $\dot{M}_{\text {acc }}$ values used for low-mass stars $\left(<0.3 M_{\odot}\right)$ were based on modelling the $\mathrm{H} \alpha$ line profile (e.g. Muzerolle et al. 2001, 2003), whereas those for higher mass stars were mainly based on spectral veiling measurements (e.g. Gullbring et al. 1998).

In Fig. 9 the $\dot{M}_{\text {acc }}-\mathrm{WH} \alpha(10 \%)$ scatter plot for the Lupus sample is compared with the Natta et al. (2004) relationship, represented with a continuous line. For objects with $\mathrm{WH} \alpha(10 \%)<400 \mathrm{~km} \mathrm{~s}^{-1}$, the Natta et al. (2004) relation tends to underestimate $\dot{M}_{\text {acc }}$ with respect to our determinations from continuum-excess modelling by $\sim 0.6 \mathrm{dex}$ in $\log \dot{M}_{\mathrm{acc}}$ on average, but the differences may be up to about an order of magnitude. Similar differences were seen in the Herczeg \& Hillenbrand (2008) Taurus sample and in the Fang et al. (2013, see their Fig. 45) sample in the L1641 cloud. For objects with 
A\&A 561, A2 (2014)

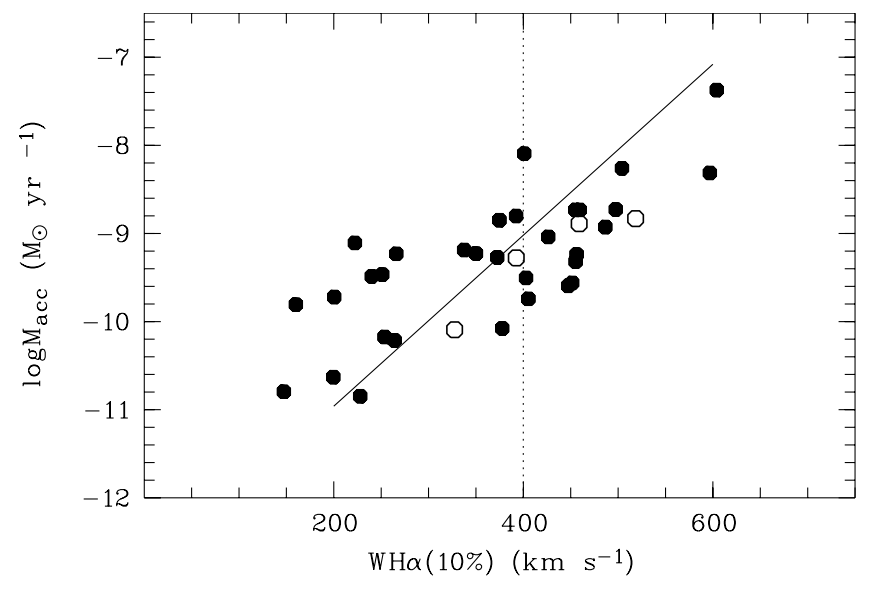

Fig. 9. $\dot{M}_{\text {acc }}$ vs. WH $\alpha(10 \%)$ for the Lupus YSOs. Symbols are the same as in Fig. 3. The $\mathrm{WH} \alpha(10 \%)$ measures are reported in Table 3. The $\dot{M}_{\text {acc }}$ values of the subluminous objects were corrected, as discussed in Sect. 7.4. The continuous line represents the relationship $\log \left(\dot{M}_{\text {acc }}\right)=$ $9.7 \times 10^{-3} \cdot \mathrm{WH} \alpha(10 \%)-12.89$ by Natta et al. (2004).

$\mathrm{WH} \alpha(10 \%)>400 \mathrm{~km} \mathrm{~s}^{-1}$, the slope of the Lupus correlation seems rather consistent with the Natta et al. (2004) relationship, but the $\log \dot{M}_{\text {acc }}$ values are systematically lower by $\sim 0.5-0.7 \mathrm{dex}$. According to the Natta et al. (2004) relationship, YSOs with WH $\alpha(10 \%)<400 \mathrm{~km} \mathrm{~s}^{-1}$ should have $\dot{M}_{\text {acc }}<10^{-9} M_{\odot} \mathrm{yr}^{-1}$, and from Fig. 8, these correspond to objects with $M_{\star}<0.3 M_{\odot}$, that is, those for which $\dot{M}_{\text {acc }}$ comes from modelling of the $\mathrm{H} \alpha$ line profile in Natta et al. (2004). Therefore, the differences we observe between $\dot{M}_{\text {acc }}$ calculated from continuum excess and those derived from the Natta et al. (2004) relationship can be attributed to the different methodologies adopted in measuring $\dot{M}_{\text {acc }}$. In conclusion, although the $\mathrm{H} \alpha$ line is the strongest in optical spectra of YSOs, special attention should be paid when using it to estimate mass accretion rates from the line width.

The least scattered $L_{\text {acc }}-L_{\text {line }}$ relations are those of the Balmer lines with $n>3$, the Br $\gamma$ line, and the He I lines. The $\operatorname{Pa} \beta$ and the $\operatorname{Br} \gamma$ relations are recommended because these lines are the least affected from chromospheric emission. In contrast, the Ca II IRT relations are the most scattered. Even after correcting for the photospheric absorption, the Ca II IRT relations appear to be more scattered than any of the Balmer lines, mainly because in some YSOs with very broad lines (e.g. Sz 83, Sz 72, Sz 69 and Lup 713) the blending with the $\mathrm{Pa} 13, \mathrm{~Pa} 15$, and $\mathrm{Pa} 16$ lines contributes to the integrated flux, increasing the line luminosity. Without correcting for the photospheric contribution, the scatter in the CaII IRT relations would be even larger. As in Mohanty et al. (2005), we also investigated relations between

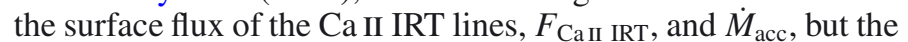
scatter remains rather large, on the order of $0.6 \mathrm{dex}$. In addition to the problem of blending with the Paschen lines, uncertainties in stellar radius and distance make the surface flux relations very scattered and uncertain. Moreover, no evidence is found in our data for a two-mode $F_{\text {Ca II IRT }}-\dot{M}_{\text {acc }}$ relation depending on the mass range, in contrast to the suggestion by Mohanty et al. (2005). This casts some doubts on whether the two Mohanty et al. (2005) relations may be produced by the different methodologies used in calculating $\dot{M}_{\text {acc }}$. In fact, the $\dot{M}_{\text {acc }}$ values for six of the eight objects used by Mohanty et al. (2005) to derive the "low-mass" relation come from modelling of the $\mathrm{H} \alpha$ line profile, while their "high-mass" relation is entirely based on veiling estimates. Note that Mohanty et al. (2005) briefly mention this as a possible reason for their different $\dot{M}_{\text {acc }}$ at their low- and highmass regimes.

Another important aspect to be considered when determining accretion rates from emission lines and $L_{\mathrm{acc}}-L_{\text {line }}$ relationships is the contribution of chromospheric emission. The relative importance of (hydrogen) line emission with respect to $L_{\text {acc }}$ is higher for low $L_{\text {acc }}$ values, and chromospheric emission may be the dominant process in the lines. Based on the luminosity of several chromospheric emission lines in the class III templates, Manara et al. (2013a) determined a threshold below which chromospheric emission dominates line luminosities. The threshold depends on YSO effective temperature and age. Line luminosities yielding $L_{\text {acc }}$ values below or just above that threshold should not be considered as accretion diagnostics.

Finally, as in Rigliaco et al. (2012), we stress that the average $L_{\text {acc }}$ derived from several lines, measured simultaneously, has a much reduced error.

\subsection{Discrepancies with magnetospheric accretion models}

The most extensive calculations of line emission in the context of magnetospheric accretion models remain those of Muzerolle et al. (2001); they were performed for the stellar parameters typical of T Tauri stars $\left(M_{\star}=0.5 M_{\odot}, R_{\star}=2 R_{\odot}\right)$, mass accretion rates between $10^{-6}$ and $10^{-9} M_{\odot} / \mathrm{yr}$, different disc truncation radii, and a wide range of gas temperatures. Their predictions of the dependence of the line luminosity and line ratios are at odds with the observed trends: namely, while the observed line luminosities increase roughly linearly with $\dot{M}_{\text {acc }}$, the models, which include constraints on the gas temperature, predict that the line luminosity will remain constant above $\dot{M}_{\text {acc }} \sim 10^{-8} M_{\odot} / \mathrm{yr}$. Muzerolle et al. proposed that the observations can only be understood if both $\dot{M}_{\text {acc }}$ and the line fluxes are controlled by the size of the accretion flow, instead of reflecting the physical conditions of the accreting gas. In other words, if the flow occurs along discrete separate magnetic flux tubes, the gas physical conditions in each tube are similar, but the number of them can increase by an order of magnitude from object to object. We confirm the trend and show that it extends over a very wide range of $\dot{M}_{\text {acc}}$.

The similarity of the physical conditions in the accreting gas for objects with very different $\dot{M}_{\text {acc }}$ is suggested by a number of other properties as well. One is the fact that the hydrogen line ratios remain quite constant over a wide range of $\dot{M}_{\text {acc }}$, and that there is no indication that optical depth effects play a significant role. An example is the $\mathrm{Pa} \beta / \mathrm{Br} \gamma$ ratio in Fig. 10: over a mass accretion range of 6 orders of magnitude, this ratio is constant within the uncertainties in the range 3-5, with no evidence for the lines to become optically thin at low $\dot{M}_{\text {acc }}$ (e.g. Muzerolle et al. 2001). We note that there are no objects with $\mathrm{Pa} \beta / \mathrm{Br} \gamma \sim 2$ in our sample, as found in some $\rho$-Oph brown dwarfs by Gatti et al. (2006). It is possible that the physical conditions in the younger and brighter BDs in $\rho$-Oph differ from those in Lupus; however, it would be worthwhile to confirm the Gatti et al. (2006) results using spectra of the same quality as the ones used here.

Another indication for similar physical conditions in the accreting gas comes from considering the relations between $L_{\text {line }}$ and $L_{\text {acc }}$ derived in Sect. 5 . To zero order they are linear, with slopes varying between 0.99 and 1.18 for the hydrogen Balmer, Paschen and Brackett lines, all equal within the errors; similarly for the He I lines, with slopes in the range 0.90-1.16. This means that over a range of $L_{\text {acc }}$ of 5 orders of magnitude, a similar fraction of the accretion energy is emitted in each line, independently of their excitation potential and optical depth. Even 


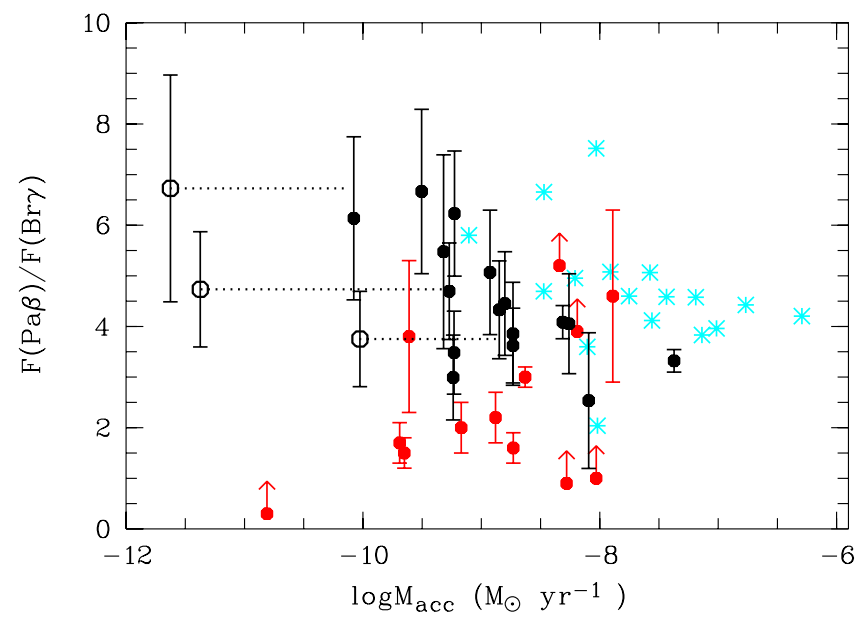

Fig. 10. $\mathrm{Pa} \beta / \mathrm{Br} \gamma$ ratio for the 19 YSOs in which both lines are detected (black dots and open circles). The shift in $\dot{M}_{\text {acc }}$ to the right for the low-luminosity YSOs (open symbols) after correcting for obscuration as explained in Sect. 7.4 is represented with the horizontal dotted lines. Objects in the $\rho$-Oph cloud studied by Gatti et al. (2006) are represented with red dots, while Taurus T Tauri stars from Muzerolle et al. (2001) are represented with cyan asterisks.

if these fractions are in most cases very small (less than $1 \%$ in the case of the hydrogen lines and less than $0.1 \%$ for the He I lines, with only $\mathrm{H} \alpha$ reaching $\sim 5 \%$ of $L_{\mathrm{acc}}$ ), they are surprisingly stable across the examined stellar parameters. This, again, suggests that the physical conditions of the accreting gas are very similar in all objects, and that only the covering factor changes from object to object.

To our knowledge, the suggestion by Muzerolle et al. (2001) that the geometry of the accretion flow controls the rate at which the disc material accretes onto the central star has not been followed-up by detailed models that include, for example, complex magnetic-field configurations.

\subsection{Accretion properties of Lupus YSOs}

Using simplified stellar evolution calculations for stars that are subject to time-dependent accretion, Tilling et al. (2008) concluded that the $L_{\mathrm{acc}}-L_{\star}$ plane presents two main features, the $L_{\text {acc }} \approx L_{\star}$ upper boundary, and diagonal tracks, which can be ascribed to accretion rates as the stars descend the Hayashi tracks. The authors showed that the slope of these tracks on the $L_{\text {acc }}-L_{\star}$ plane is related to the power-law index of the $\dot{M}_{\text {acc }}$ vs. age relationship. Our sample is consistent with an $L_{\text {acc }} \propto L_{\star}{ }^{1.7}$ law, and under the Tilling et al. (2008) prescription, where $L_{\text {acc }} \propto L_{\star}(\eta-0.3) / 0.7$ (see their equation 12), this would mean $\eta=1.5$, leading to $\dot{M}_{\text {acc }} \propto t^{-1.5}$

A detailed observational study of the evolution of accretion requires complete and homogeneous samples of YSOs, with a diversity of ages and well-determined $\dot{M}_{\text {acc }}$ (cf. Sicilia-Aguilar et al. 2010). Gatti et al. (2008) found that the accretion rates are significantly lower in $\sigma$-Ori than in younger regions, such as $\rho$-Oph, which is consistent with viscous disc evolution. Manara et al. (2012) and Rigliaco et al. (2011a) discussed the evolution of accretion as a function of stellar mass in the ONC and the $\sigma$-Ori cluster, respectively. The age range of our sample is too narrow to investigate the $\dot{M}_{\text {acc }} \propto t^{-\eta}$ relation in detail, but selecting the objects in the mass range $0.4 M_{\odot}$ to $0.8 M_{\odot}$, and excluding subluminous objects, an attempt to fit the relation yields $\eta=1.3 \pm 0.3$, albeit with a large dispersion. The average $\dot{M}_{\text {acc }}$ for objects in the same mass range is $1.3\left({ }_{-0.8}^{+1.9}\right) \times 10^{-9} M_{\odot} \mathrm{yr}^{-1}$, which is consistent with the calculations of the evolution of viscous discs for $0.5 M_{\odot}$ YSOs reported by Hartmann et al. (1998) at $3 \mathrm{Myr}$ (see their Fig. 3). To empirically investigate the $\dot{M}_{\text {acc }}$ vs. age relationship, Caratti o Garatti et al. (2012) normalised the $\dot{M}_{\text {acc }}$ to $M_{\star}{ }^{2}$ to account for the dependence of $\dot{M}_{\text {acc }}$ on mass. Following this approach for the Lupus YSOs, some number statistics is gained by including in the fit YSOs with mass of up to $\sim 1 M_{\odot}$. Although the dispersion is slightly reduced, the result is practically identical.

Since the stellar mass undergoes negligible changes during the class II phase, the $\dot{M}_{\text {acc }}$ vs. $M_{\star}$ relation represents a diagnostic tool for the evolution of $\dot{M}_{\text {acc }}$ (Clarke \& Pringle 2006). From the theoretical point of view, it has been suggested by Vorobyov \& Basu (2008) that the $\dot{M}_{\text {acc }} \propto M_{\star}^{2}$ relationship can be explained on the basis of self-regulated accretion by gravitational torques in self-gravitating discs. These authors argued that the relationship can be better described as a double power-law, with the break occurring at $M_{\star} \approx 0.25 M_{\odot}$, instead of as a function with a single exponent (Vorobyov \& Basu 2009). Interestingly, such a kink occurs close to the value of mass where the techniques of calculating $\dot{M}_{\text {acc }}$ differ. Thus, they also point out the different techniques used in determining $\dot{M}_{\text {acc }}$ as a possible cause for the apparent bi-modal power-law.

The power-law index of the $\dot{M}_{\mathrm{acc}}-M_{\star}$ relation for Lupus is also $\sim 2$, but the scatter is smaller than in previous data sets (cf. the standard deviation for the Lupus fit is a factor 2 lower than for the Taurus sample in Herczeg \& Hillenbrand 2008). As pointed out in Sect. 2, our sample represents about $50 \%$ of the complete population of class II YSOs in the Lupus I and Lupus III clouds. It is therefore unlikely that the wide range of $\dot{M}_{\text {acc }}(>2$ dex) at a given mass observed in other data sets will be also detected in Lupus, in a more complete sample. However, it would be worthwhile to study YSOs more massive than those in our sample, using spectra of the same quality as we did. In addition, although our number statistics is low, there is no evidence for a double power-law in our sample, supporting the conclusion that the apparent bi-modal relations suggested in the literature between $\dot{M}_{\text {acc }}$ and other YSOs properties are probably the result of mixing $\dot{M}_{\text {acc }}$ derived with different methods.

\subsection{Subluminous YSOs: evolution or edge-on discs?}

The subluminosity and strong emission lines in the optical spectrum of Par-Lup3-4 were first discussed by Comerón et al. (2003), who found it difficult to explain their observations in terms of either a photospheric continuum suppressed by an edgeon disc or an embedded source seen in scattered light. They favoured instead the scenario in which the PMS evolution is significantly modified by the accretion process, as suggested in Baraffe \& Chabrier (2010). The analysis of the SED resulting in a disc inclination angle of $\sim 81^{\circ}$ (Huelamo et al. 2010) and the tiny difference in velocity between the red- and blue-shifted components of the outflow (Bacciotti et al. 2011; Whelan et al. 2013) provide evidence that we are seeing the Par-Lup3-4 disc almost edge-on. However, as in Comerón et al. (2003), strong Ca II IRT lines are also detected in our X-shooter spectrum, meaning that not all the emission from the accretion flows in the inner regions is suppressed by the optically thick disc.

Comerón et al. (2003) also pointed out that Sz 106 and Sz 113 are subluminous, although not as extremely. Subluminosity is confirmed here for $\mathrm{Sz} \mathrm{106,} \mathrm{while} \mathrm{no} \mathrm{evidence}$ 
for this phenomenon is observed in $\mathrm{Sz} 113^{9}$. We also find Lup 706 and Sz 123B to be subluminous, but not by as much as Par-Lup3-4.

As examined by Baraffe \& Chabrier (2010), episodic strong accretion during the PMS evolution of low-mass stars produces objects with a smaller radius, a higher central temperature, and a lower luminosity than the non-accreting counterpart of the same mass and age, resulting in low-luminosity objects. If the PMS evolution of the four subluminous YSOs had been significantly altered by episodic strong accretion, their surface gravity would also appear higher than for objects of the same mass and age. One way to test this possibility is through independent estimates of the surface gravity. We achieved these estimates while correcting the photospheric contribution of the $\mathrm{Na}$ I D and Ca II IRT lines (see Sect. 4.4), when using the gravity sensitive Na I $(\lambda \lambda 818.3 \mathrm{~nm} / 819.48 \mathrm{~nm})$ and the K I $(\lambda \lambda 766.50 \mathrm{~nm} / 769.90 \mathrm{~nm})$ doublets (see Stelzer et al. 2013b, for the method), for which veiling is negligible in these objects. The results of the analysis yield $\log g$ values $(3.5,3.7,4.3$, and 3.9 for Par-Lup3-4, Lup 706, Sz 106 and Sz 123B, respectively) that are very similar to those of the other YSOs in the sample, and are lower than those expected for apparently more evolved objects. Hence, we reject the hypothesis that the subluminosity of these objects is the result of modified PMS evolution by accretion episodes.

The scatter in several plots shown throughout the previous sections is significantly reduced when $L_{\text {acc }}$ is normalised to the YSOs luminosity; the $L_{\mathrm{acc}} / L_{\star}$ values of the subluminous objects are consistent with those for the other YSOs, suggesting that some process is active that affects $L_{\text {acc }}$ and $L_{\star}$ in the same way. As in the case of Par-Lup3-4, such a process may be gray circumstellar extinction. In the magnetospheric accretion model, the line- and accretion luminosities originate in the inner parts of the star-disc system. Thus, under the assumption of gray circumstellar extinction due to disc obscuration, $L_{\text {acc }}$ and $L_{\text {line }}$ as well as the YSO luminosity are dimmed by the same amount, which depends on disc inclination angle, disc flaring, and dust opacity. The effect therefore cancels out when considering the $L_{\mathrm{acc}} / L_{\star}$ ratio and has no consequence for the $L_{\text {acc }}$ vs. $L_{\text {line }}$ relationships, but may have a considerable impact on the $\dot{M}_{\text {acc }}$ determinations for the most obscured objects.

Under the hypothesis of a gray obscuration, it can be inferred from Fig. 3 that the obscuration factors by which the luminosity of the four objects should be multiplied to fit the average trend of the other YSOs are $\sim 25,10,6$, and 4 for Par-Lup3-4, Lup706, Sz 123B, and Sz 106, respectively. At a fixed mass, Eq. (1) implies $\dot{M}_{\text {acc }} \propto L_{\text {acc }} \cdot L_{\star}{ }^{0.5}$, because the YSO radius scales with the square root of the luminosity. Assuming that the obscuration factor suppresses both $L_{\text {acc }}$ and $L_{\star}$ by the same amount, the $\dot{M}_{\text {acc }}$ values for the low-luminosity YSOs can be corrected as $\dot{M}_{\text {acc }}($ corrected $)=(\text { obscuration factor })^{1.5} \dot{M}_{\text {acc }}$. When the obscuration factors are applied to $L_{\star}, L_{\text {acc }}$ and $\dot{M}_{\text {acc }}$, the four subluminous objects behave exactly as the other YSOs in the various plots. A detailed analysis of the SED of these YSOs is required to constrain the geometry of their disc.

\footnotetext{
9 We noted some differences between our spectra of Sz 106 and Sz 113 and those published by Comerón et al. (2003). For instance, the $\mathrm{H} \alpha$ equivalent width we measure for Sz 106 is $11.6 \AA$ A, while Comerón et al. (2003) claimed more than $100 \AA$. Moreover, lots of forbidden lines are seen in our spectrum of Sz 113, while Comerón et al. (2003) detected none. Note, however, that the Comerón et al. (2003) spectra have a much lower resolution than ours.
}

\section{Summary and conclusions}

Our study with X-shooter@VLT is the first that presents UV-excess measures of accretion luminosity simultaneously with intermediate-resolution spectroscopy of a large number of emission line diagnostics from $\sim 330 \mathrm{~nm}$ to $2500 \mathrm{~nm}$ in a significant and homogeneous sample of very low mass YSOs in Lupus. The quality of the spectra and the accuracy in flux calibration, both in the continuum and the lines, allowed the characterisation of the sample and the computation of $L_{\mathrm{acc}}$ vs. $L_{\text {line }}$ relations for an unprecedentedly large number (39) of emission line diagnostics, as well as to study the accretion properties of the sample. The main results are summarised here.

The accretion emission in our sample is dominated by continuum emission in the Balmer and Paschen continuum. For the vast majority of the YSOs the integrated line luminosity is lower than one third of $L_{\mathrm{acc}}$. Most of the line luminosity is due to Balmer lines, yet the contribution of the Paschen, Brackett, and other permitted lines in the strongest accretors may be similar to the total Balmer line luminosity. The accretion level of all the YSOs studied here is well above the expected chromospheric contribution, even at the lowest values of $L_{\mathrm{acc}}$.

The 39 empirical $L_{\text {acc }}$ vs. $L_{\text {line }}$ relationships computed here have in general a lower dispersion than previously reported relationships in the literature. Our $L_{\text {acc }}$ vs. $L_{\text {line }}$ relationships agree well with previous results, but systematic differences may exist at different mass regimes with respect to studies that adopted other methodologies of measuring $L_{\text {acc }}$ or $\dot{M}_{\text {acc }}$. We confirm that for low-mass YSOs $\left(M_{\star}<0.3 M_{\odot}\right), \mathrm{H} \alpha$ line profile modelling may underestimate $\dot{M}_{\text {acc }}$ by 0.6 to $0.8 \mathrm{dex}$ with respect to $\dot{M}_{\text {acc }}$ derived from continuum-excess measures.

The least scattered among our $L_{\text {acc }}-L_{\text {line }}$ relationships are those for the Balmer lines $n>3$, the $\mathrm{Br} \gamma$ line, and the He I lines. The $\operatorname{Pa} \beta$ and the $\operatorname{Br} \gamma$ relations are recommended because they are less affected by chromospheric activity than the optical lines. The most scattered relations are those of the Ca II IRT. Likewise, the Ca II IRT surface-flux relationships have large scatter $(\sim 0.6$ dex $)$, mainly because of the blending and contribution of Paschen lines in strong accretors and to the uncertainties on stellar parameters. The previously suggested bi-modality with respect to the mass of the Ca II IRT surface-flux relationships is most likely induced by the mix of different methodologies employed in deriving $\dot{M}_{\text {acc }}$. More generally, we conclude that mixing mass-accretion rates calculated with different techniques may lead to a spurious bi-modality in the relationships between $\dot{M}_{\text {acc }}$ and YSOs properties. The average $L_{\text {acc }}$ derived from several lines, measured simultaneously, has a significantly reduced error.

The accretion properties of the YSOs studied here are similar to those of other low-mass YSOs in regions such as Taurus, $\rho$-Oph or $\sigma$-Ori. We derived $\dot{M}_{\text {acc }}$ in the range from $2 \times 10^{-12}$ to $4 \times 10^{-8} M_{\odot} \mathrm{yr}^{-1}$ for objects with masses from 0.03 to $1.2 M_{\odot}$. We conclude that $\dot{M}_{\text {acc }} \propto M_{\star}{ }^{1.8( \pm 0.2)}$ for the Lupus sample studied here, in agreement with most studies of the $\dot{M}_{\text {acc }}-M_{\star}$ relationship. The scatter for the Lupus relationship is smaller than for other data sets. The average $\dot{M}_{\text {acc }}$ for objects with mass between 0.4 and $0.8 M_{\odot}$ is consistent with the calculations of the evolution of $\dot{M}_{\text {acc }}$ in viscous discs for 3 Myr old objects with $0.5 M_{\odot}$ (Hartmann et al. 1998).

We confirmed and extended over more than 5 orders of magnitude in $\dot{M}_{\text {acc }}$ some properties of the accretion emission that were known for a more limited range of $\dot{M}_{\text {acc }}$. In particular, line ratios, as well as the fraction of $L_{\text {acc }}$ emitted in each line, are roughly independent of $\dot{M}_{\text {acc }}$, and the line luminosities 
increase almost linearly with $\dot{M}_{\text {acc }}$ over the whole range. This suggests that some inconsistencies between magnetospheric accretion models and observations still prevail, but a number of properties (e.g. constant hydrogen line ratios, same accretion budget emitted in each line independently of optical depth, and linear correlation of the line luminosity with $\dot{M}_{\text {acc }}$ ) suggest that the physical conditions of the accreting gas are similar over a wide range of $\dot{M}_{\text {acc }}$. Our data show that these properties are valid over a wide range in $\dot{M}_{\text {acc }}$, extending to the very low mass regime. We thus confirm the suggestion by Muzerolle et al. (2001) that the geometry of the accretion flows controls the rate at which the disc material accretes onto the central star: higher mass accretion rates require larger emitting areas. Detailed magnetospheric accretion models, incorporating complex magnetic field topologies, are needed to understand whether other physical parameters (e.g. magnetic-field topology) play a role in the accretion physics.

Acknowledgements. We thank the anonymous referee for her/his careful reading and for suggestions. J.M.A., E.C., B.S., and K.B. thank G. Attusino for stimulating discussions. We also thank B. Nisini, T. Giannini and S. Antoniucci for lively discussions. We thank V. D'Odorico, P. Goldoni and A. Modigliani for their help with the X-shooter pipeline, and F. Getman and G. Capasso for the installation of the different pipeline versions at Capodimonte. We also thank the ESO staff, in particular F. Patat, for suggestions in OB preparation and C. Martayan for support during the observations. Financial support from INAF is also acknowledged. This research made use of the SIMBAD database, operated at the CDS (Strasbourg, France). This publication makes use of data products from the Two Micron All Sky Survey, which is a joint project of the University of Massachusetts and the Infrared Processing and Analysis Center/California Institute of Technology, funded by NASA and the National Science Foundation. This publication makes use of data products from the Wide-field Infrared Survey Explorer, which is a joint project of the University of California, Los Angeles, and the Jet Propulsion Laboratory/California Institute of Technology, funded by the National Aeronautics and Space Administration.

\section{References}

Alcalá, J. M., Stelzer, B., Covino, E., et al. 2011, AN, 332, 242 Alexander, R. D., Clarke, C. J., \& Pringle, J. E. 2006, MNRAS, 369, 216 Allard, F., Homeier, D., \& Freytag, B. 2012, in 16th Cambridge Workshop on Cool Stars, Stellar Systems, and the Sun, eds. C. M. Johns-Krull, et al., ASP Conf Ser., 448, 91

Allen, P. R., Luhman, K. L., Myers, P. C., et al. 2007, ApJ, 655, 1095

Allers, K. N., Kessler-Silacci, J. E., Cieza, L. A., \& Jaffe, D. T. 2006, ApJ, 644, 364

Antoniucci, S., Garcia Lopez, R., Nisini, B., et al. 2011, A\&A, 534, A32

Appenzeller, I., Jankovics, I., \& Krautter, J. 1983, A\&AS, 53, 291

Baraffe, I., \& Chabrier, G. 2010, A\&A, 521, A44

Baraffe, I., Chabrier, G., Allard, F., et al. 1998, A\&A, 337, 403

Bacciotti, F., Whelan, E. T., Alcalá, J. M., et al. 2011, ApJ, 737, 26

Bayo, A., Barrado, D., Huélamo, N., et al. 2012, A\&A, 547, A80

Biazzo, K., Alcalá, J. M., Covino, et al. 2012, A\&A, 547, A104

Calvet, N., \& Gullbring, E. 1998, ApJ, 509, 802

Calvet, N., Hartmann, L., \& Strom, S. E. 2000, in Protostars and Planets IV, 377

Calvet, N., Muzerolle, J., Briceño, C., et al. 2004, AJ, 128, 1294

Caratti o Garatti, A., García López, R., Antoniucci, S., et al. 2012, A\&A, 538, A64

Chabrier, G., Baraffe, I., Allard, F., \& Hauschildt, P. 2000, ApJ, 542, 464

Clarke, C, J., \& Pringle, J. E. 2006, MNRAS, 370, L10

Comerón, F. 2008, in Handbook of Star Forming Regions, Volume II: The Southern Sky ASP Monograph Publications, ed. Bo Reipurth, 5, 295

Comerón, F., Fernández, M., Baraffe, I., Neuhäuser, R., \& Kaas, A. 2003, A\&A, 406, 1001

Comerón, F., Spezzi, L., \& López-Martí, B. 2009, A\&A, 500, 1045

Comerón, F., Testi, L., \& Natta, A. 2010, A\&A, 522, A47

Costigan, G., Scholz, A., Stelzer, B., et al. 2012, MNRAS, 427, 1344

Costigan, G., Vink, J. S., Scholz, A., Ray, T., \& Testi, L. 2013, MNRAS, submitted

Cutri, R. M., Skrutskie, M. F., van Dyk, S., et al. 2003, 2MASS All Sky Catalog of Point Sources, The IRSA 2MASS All-Sky Point Source Catalog, NASA/IPAC Infrared Science Archive (Pasadena, CA: NASA/IPAC), http://irsa.ipac. caltech. edu/application/Gator
D’Antona, F., \& Mazzitelli, I. 1997, Mem. SAIt., 68, 807

Draine, B. T. 2003, ARA\&A, 41, 241

Dullemond, C. P., \& Dominik, C. 2004, A\&Ap, 417, 159

Edwards, S., Fischer, W., Hillenbrand, L., \& Kwan, J. 2006, ApJ, 646, 319

Evans, N. J., II, Dunham, M. M., Jørgensen, J. K., et al. 2009, ApJS, 181, 321

Fang, M., van Boekel, R., Wang, W., et al. 2009, A\&A, 504, 461

Fang, M., Jinyoung, S., van Boekel, R., et al. 2013, ApJS, 207, 5

Feigelson, E. D., \& Nelson, P. I. 1985, ApJ, 293, 192

Fernández, M., \& Comerón, F. 2001, A\&A, 380, 264

Frasca, A., Guillout, P., Marilli, E., et al. 2006, A\&A, 454, 301

Gahm, G. F., Walter, F. M., Stempels, H. C., Petrov, P. P., \& Herczeg, G., 2008,

A\&A, 482, 35

Gatti, T., Testi, L., Natta, A., et al. 2006, A\&A, 460, 547

Gatti, T., Natta, A., Randich, S., et al. 2008, A\&A, 481, 423

Giannini, T., Nisini, B., Antoniucci, S., et al. 2013, ApJ, 778, 71

Gorti, U., \& Hollenbach, D. 2009, ApJ, 690, 1539

Graham, J. A., \& Heyer, M. H. 1988, PASP, 100, 1529

Gullbring, E., Hartmann, L., Briceño, C., \& Calvet, N. 1998, ApJ, 492, 323

Guenther, E. W., Esposito, M., Mundt, R., et al. 2007, A\&A, 467, 1147

Hartigan, P., \& Kenyon, S. J. 2003, ApJ, 583, 334

Hartigan, P., Kenyon, S. J., Hartmann, L., et al. 1991, ApJ, 382, 617

Hamann, F., \& Persson, S. E. 1992, ApJS, 82, 247

Hartmann, L. 1998, Accretion Processes in Star Formation (Cambridge Univ. Press)

Hartmann, L. E., Hewett, R., \& Calvet, N. 1994, ApJ, 426, 669

Hartmann, L. E., Calvet, N., Gullbring, E., \& D’Alessio, P. 1998 ApJ, 495, 385

Hauschildt, P., Allard, F., \& Baron, E. 1999a, ApJ, 512, 277

Hauschildt, P., Allard, F., Ferguson, J., et al. 1999b, ApJ, 525, 871

Herbig, G. H., \& Soderblom, D. R. 1980 ApJ, 242, 628

Herczeg, G., \& Hillenbrand, L. A. 2008, ApJ, 681, 594

Herczeg, G., Linsky, J., Valenti, J., et al. 2002, ApJ, 572, 310

Herczeg, G., Walter, F. M., Linsky, J. L., et al. 2005, AJ, 129, 2777

Herczeg, G., Cruz, K. L., \& Hillenbrand, L. A. 2009, ApJ, 696, 1589

Huélamo, N., Bouy, H., Pinte, C., et al. 2010, A\&A, 523, A42

Hughes, J., Hartigan, P., Krautter, J., \& Kelemen, J. 1994, AJ, 108, 1071

Ingleby, L., Calvet, N., Bergin, E., et al. 2011, ApJ, 743, 105

Ingleby, L., Calvet, N., Herczeg, G., et al. 2013, ApJ, 767,112

Johns-Krull, C. M. 2007, ApJ, 664, 975

Kenyon, S., \& Hartmann, L. 1987, ApJ 323, 714

Kenyon, S., \& Hartmann, L. 1995, ApJ, 101, 117

Königl, A. 1991, ApJ, 370, L39

Kurosawa, R., Harries, T. J., \& Symingtong, N. H. 2006, MNRAS, 370, 580

López-Martí, B., Eislöffel, J., \& Mundt, R., A\&A, 440, 139

Luhman, K. L., Stauffer, J., Muench, A., et al. 2003, ApJ, 593, 1093

Luhman, K., Adame, L., D’Alessio, P., et al. 2005, ApJ, 635, 93

Lynden-Bell, D., \& Pringle, J. E. 1974, MNRAS, 168, 603

Manara, C. F., Robberto, M., Da Rio, N., et al. 2012, ApJ, 755, 154

Manara, C., Testi, L., Rigliaco, E., et al. 2013a, A\&A, 551, A107

Manara, C., Beccari, G., Da Rio, N., et al. 2013b, A\&A, 558, A114

Melo, C. 2003, A\&A, 410, 269

Merín, B., Jørgensen, J. K., Spezzi, L., et al. 2008, ApJS, 177, 551

Modigliani, A., Goldoni, P., Royer, F., et al. 2010, in Observatory Operations: Strategies, Processes, and Systems III, eds. D. R. Silva, A. B. Peck, \& B. T. Soifer, SPIE, 7737

Mohanty, S., Jayawardhana, R., \& Basri, G. 2005, ApJ, 626, 498

Mohanty, S., Greaves, J., Mortlock, D., et al. 2013, ApJ, in press

Mortier, A., Oliveira, I., \& van Dishoeck, E. F. 2011, MNRAS, 418, 1194

Muzerolle, J., Hartmann, L., \& Calvet, N. 1998, AJ, 116, 455

Muzerolle, J., Calvet, N., Briceño, C., Hartmann, L., \& Hillenbrand, L. 2000, ApJ, 535, L47

Muzerolle, J., Calvet, N., \& Hartmann, L. 2001, ApJ, 550, 944

Muzerolle, J., Hillenbrand, L., Calvet, N., et al. 2003, ApJ, 592, 266

Muzerolle, J., Luhman, K., Briceño, C., et al. 2005, ApJ, 625, 906

Natta, A., Testi, L., Muzerolle, J., et al. 2004, A\&A, 424, 603

Natta, A., Testi, L., \& Randich, S. 2006, A\&A, 452, 245

Nisini, B., Bacciotti, F., Giannini, et al. 2005, A\&A, 441, 159

Olofsson, J., Augereau, J.-C., van Dishoeck, E. F., et al. 2010, A\&A, 520, A39

Olofsson, J., Szucs, L., Henning, Th., et al. 2013, A\&A, in press, DOI: 10.1051/0004-6361/201321967

Paatz, G., \& Camenzind, M. 1996, A\&A, 308, 77

Pinte, C., Padgett, D. L., Ménard, F., et al. 2008, A\&Ap, 489, 633

Pringle, J. E. 1991, MNRAS, 248, 754

Rajpurohit, A. S., Reylé, C., Allard, F., Homeier, D., Schultheis, M. et al. 2013, A\&A, 556, A15

Reipurth, B., Bally, J., Graham, J. A., et al. 1986, A\&A, 164, 51

Ricci, L., Testi, L., Natta, A., et al. 2010, A\&A, 512, A15

Riddick, F., Roche, P., \& Lucas, P. 2007, MNRAS, 381, 1067

Rigliaco, E., Natta, A., Randich, S., et al. 2011a, A\&A, 525, A47 
A\&A 561, A2 (2014)

Rigliaco, E., Natta, A., Randich, S., et al. 2011b, A\&A, 526, L6 Rigliaco, E., Natta, A., Testi, L., et al. 2011c, AN, 332, 249 Rigliaco, E., Natta, A., Testi, L., et al. 2012, A\&A, 548, A56

Rygl, K. L. J., Benedettini, M., Schisano, E., et al. 2013, A\&A, 549, 1

Rojas-Ayala, B., Covey, K. R., Muirhead, P. S., \& Lloyd, J. P. 2012, ApJ, 748, 93

Scholz, A., Jayawardhana, R., Wood, K., et al. 2007, ApJ, 660, 1517 Sicilia-Aguilar, A., Henning, Th., \& Hartmann, L. W. 2010, ApJ, 710, 597

Shu, F., Najita, J., Ostriker, E., \& Wilkin, F. 1994, ApJ, 429, 781

Spezzi, L., Alcalá, J. M., Covino, E., et al. 2008, ApJ, 680, 1295

Stelzer, B., Alcalá, J. M., Biazzo, K., et al. 2012, A\&A, 537, A94

Stelzer, B., Alcalá, J. M., Scholz, A., et al. 2013, A\&A, 551, A106

Stelzer, B., Frasca, A., Alcalá, J. M., et al. 2013b, A\&A, 558, A141

Testi, L. 2009, A\&A, 503, 639
Tillling, I., Clarke, C. J., Pringle, J. E., \& Tout, C. A. 2008, MNRAS, 385, 1530

Uchida, Y.. \& Shibata, K. 1985, PASJ, 37, 515

Valenti, J. A., Basri, G., \& Johns, C. M. 1993, ApJ, 106, 2024

Vernet, J., Dekker, H., D’Odorico, S., Kaper, L., et al. 2011, A\&A, 536, 105

Vorovyov, E. J., \& Basu, S. 2008, ApJ, 676, 139

Vorovyov, E. J., \& Basu, S. 2009, ApJ, 703, 922

Weingartner, J. C., \& Draine, B. T. 2001, ApJ, 548, 296

Whelan, E., Bonito, R., Antoniucci, S., et al. 2013, A\&A, submitted

White, R., \& Basri, G. 2003, ApJ, 582, 1109

White, R., \& Hillenbrand, L. A. 2004, ApJ, 582, 1109

Wichmann, R., Krautter, J., Covino, E., et al. 1997, A\&A, 320, 185

Wright, E. L., Eisenhardt, P. R. M., Mainzer, A. K., et al. 2010, AJ, 140, 1868

Yang, H., Herczeg, G., Linsky, J., et al. 2012, ApJ, 744, 12 
Table 1. Selected YSOs and observing log.

\begin{tabular}{|c|c|c|c|c|c|c|c|c|c|c|c|}
\hline Object/other name & $\begin{array}{l}\mathrm{RA}(2000) \\
\mathrm{h}: \mathrm{m}: \mathrm{s}\end{array}$ & $\begin{array}{c}\operatorname{Dec}(2000) \\
\circ, \prime \prime\end{array}$ & $\begin{array}{c}\text { Obs. date } \\
\text { YY-MM-DD }\end{array}$ & $\begin{array}{c}\text { MJD } \\
(+2400000)\end{array}$ & UVB & $\frac{T_{\exp }(\mathrm{s})}{\mathrm{VIS}}$ & NIR & UVB & VIS & NIR & $\begin{array}{l}\text { Lupus } \\
\text { cloud }\end{array}$ \\
\hline $\mathrm{Sz}_{6}^{\dagger}$ & $15: 39: 28.28$ & $-34: 46: 18.0$ & 2012-04-18 & 56035.2516 & $2 \times 300$ & $2 \times 250$ & $2 \times 100$ & 40 & 140 & 55 & I \\
\hline AKC2006-19 & $15: 44: 57.90$ & $-34: 23: 39.5$ & 2011-04-23 & 55674.1966 & $2 \times 900$ & $2 \times 900$ & $2 \times 900$ & 7 & 50 & 40 & I \\
\hline Sz69 / HW Lup ${ }^{\dagger}$ & $15: 45: 17.42$ & $-34: 18: 28.5$ & 2011-04-23 & 55674.0991 & $2 \times 300$ & $2 \times 300$ & $2 \times 300$ & 12 & 70 & 60 & I \\
\hline Sz71 / GW Lup & $15: 46: 44.73$ & $-34: 30: 35.5$ & 2012-04-18 & 56035.0949 & $2 \times 300$ & $2 \times 250$ & $2 \times 100$ & 40 & 110 & 52 & I \\
\hline Sz72 / HM Lup & $15: 47: 50.63$ & $-35: 28: 35.4$ & 2012-04-18 & 56035.1926 & $2 \times 300$ & $2 \times 250$ & $2 \times 100$ & 33 & 95 & 39 & I \\
\hline Sz73 & $15: 47: 56.94$ & $-35: 14: 34.8$ & 2012-04-18 & 56035.2923 & $2 \times 300$ & $2 \times 250$ & $2 \times 100$ & 20 & 115 & 55 & I \\
\hline Sz74 / HN Lup & $15: 48: 05.23$ & $-35: 15: 52.8$ & 2012-04-18 & 56035.1695 & $2 \times 150$ & $2 \times 100$ & $2 \times 50$ & 22 & 135 & 45 & I \\
\hline Sz83 / RU Lup & $15: 56: 42.31$ & $-37: 49: 15.5$ & 2012-04-18 & 56035.1794 & $2 \times 100$ & $2 \times 50$ & $2 \times 30$ & 45 & 130 & 50 & I \\
\hline Sz84 & $15: 58: 02.53$ & $-37: 36: 02.7$ & 2012-04-18 & 56035.1083 & $2 \times 350$ & $2 \times 300$ & $2 \times 115$ & 40 & 120 & 80 & I \\
\hline Sz130 & $16: 00: 31.05$ & $-41: 43: 37.2$ & 2010-04-07 & 55293.3609 & $2 \times 300$ & $2 \times 300$ & $2 \times 300$ & 40 & 90 & 45 & IV \\
\hline Sz88A (SW) / HO Lup (SW) & 16:07:00.54 & $-39: 02: 19.3$ & 2012-04-18 & 56035.3342 & $2 \times 300$ & $2 \times 250$ & $2 \times 100$ & 60 & 110 & 50 & I \\
\hline Sz88B (NE) / HO Lup (NE) & 16:07:00.62 & $-39: 02: 18.1$ & 2012-04-18 & 56035.3342 & $2 \times 300$ & $2 \times 250$ & $2 \times 100$ & 18 & 75 & 48 & III \\
\hline Sz91 & 16:07:11.61 & $-39: 03: 47.1$ & 2012-04-18 & 56035.3213 & $2 \times 300$ & $2 \times 250$ & $2 \times 300$ & 40 & 70 & 35 & III \\
\hline Lup713 ${ }^{\dagger}$ & $16: 07: 37.72$ & $-39: 21: 38.8$ & 2010-04-06 & 55292.1950 & $2 \times 900$ & $2 \times 900$ & $2 \times 900$ & 8 & 50 & 45 & III \\
\hline Lup604s & 16:08:00.20 & $-39: 02: 59.7$ & 2010-04-06 & 55292.2374 & $2 \times 450$ & $2 \times 450$ & $2 \times 450$ & 5 & 60 & 40 & III \\
\hline Sz97 & 16:08:21.79 & $-39: 04: 21.5$ & 2011-04-23 & 55674.1139 & $2 \times 300$ & $2 \times 300$ & $2 \times 300$ & 15 & 120 & 55 & III \\
\hline Sz99 & 16:08:24.04 & $-39: 05: 49.4$ & 2010-04-07 & 55293.3452 & $2 \times 300$ & $2 \times 300$ & $2 \times 300$ & 14 & 60 & 35 & III \\
\hline $\mathrm{Sz}_{100^{\dagger}}$ & $16: 08: 25.76$ & $-39: 06: 01.1$ & 2011-04-23 & 55674.1391 & $2 \times 300$ & $2 \times 300$ & $2 \times 300$ & 10 & 50 & 55 & III \\
\hline Sz103 & $16: 08: 30.26$ & $-39: 06: 11.1$ & 2011-04-23 & 55674.1542 & $2 \times 300$ & $2 \times 300$ & $2 \times 300$ & 15 & 80 & 50 & III \\
\hline Sz104 & 16:08:30.81 & $-39: 05: 48.8$ & 2010-04-06 & 55292.2549 & $2 \times 300$ & $2 \times 300$ & $2 \times 300$ & 10 & 70 & 50 & III \\
\hline Lup706 & 16:08:37.30 & $-39: 23: 10.8$ & 2010-04-06 & 55292.2796 & $4 \times 900$ & $4 \times 900$ & $4 \times 900$ & 6 & 20 & 30 & III \\
\hline Sz106 & 16:08:39.76 & $-39: 06: 25.3$ & 2012-04-18 & 56035.3051 & $2 \times 450$ & $2 \times 400$ & $2 \times 450$ & 36 & 85 & 50 & III \\
\hline Par-Lup3-3 & 16:08:49.40 & $-39: 05: 39.3$ & 2010-04-06 & 55292.3586 & $2 \times 300$ & $2 \times 300$ & $2 \times 300$ & 5 & 50 & 30 & III \\
\hline Par-Lup3-4 & $16: 08: 51.43$ & $-39: 05: 30.4$ & 2010-04-07 & 55293.1763 & $4 \times 900$ & $4 \times 900$ & $4 \times 900$ & 7 & 30 & 45 & III \\
\hline Sz110 / V1193 Sco & 16:08:51.57 & $-39: 03: 17.7$ & 2011-04-23 & 55674.3413 & $2 \times 300$ & $2 \times 300$ & $2 \times 300$ & 35 & 103 & 55 & III \\
\hline Sz111 / Hen 3-1145 & 16:08:54.69 & $-39: 37: 43.1$ & 2012-04-18 & 56035.1229 & $2 \times 300$ & $2 \times 250$ & $2 \times 100$ & 50 & 90 & 45 & III \\
\hline Sz112 & 16:08:55.52 & $-39: 02: 33.9$ & 2012-04-18 & 56035.2644 & $2 \times 350$ & $2 \times 300$ & $2 \times 350$ & 20 & 40 & 35 & III \\
\hline Sz113 & $16: 08: 57.80$ & $-39: 02: 22.7$ & 2011-04-23 & 55674.3566 & $2 \times 900$ & $2 \times 900$ & $2 \times 900$ & 15 & 70 & 38 & III \\
\hline 2MASS J16085953-3856275 & $16: 08: 59.53$ & $-38: 56: 27.6$ & 2011-04-23 & 55674.2808 & $4 \times 900$ & $4 \times 900$ & $4 \times 900$ & 5 & 18 & 20 & III \\
\hline SSTc2d160901.4-392512 & 16:09:01.40 & $-39: 25: 11.9$ & 2011-04-23 & 55674.2260 & $2 \times 450$ & $2 \times 450$ & $2 \times 450$ & 20 & 80 & 45 & III \\
\hline Sz114 / V908 Sco & 16:09:01.84 & $-39: 05: 12.5$ & 2011-04-23 & 55674.3850 & $2 \times 300$ & $2 \times 300$ & $2 \times 300$ & 30 & 90 & 30 & III \\
\hline Sz115 & 16:09:06.21 & $-39: 08: 51.8$ & 2012-04-18 & 56035.3469 & $2 \times 350$ & $2 \times 300$ & $2 \times 350$ & 20 & 70 & 45 & III \\
\hline Lup818s ${ }^{\dagger}$ & 16:09:56.29 & $-38: 59: 51.7$ & 2011-04-23 & 55674.2442 & $2 \times 900$ & $2 \times 900$ & $2 \times 900$ & 5 & 25 & 33 & III \\
\hline Sz123A (S) & $16: 10: 51.34$ & $-38: 53: 14.6$ & 2012-04-18 & 56035.2784 & $2 \times 700$ & $2 \times 600$ & $2 \times 350$ & 25 & 60 & 45 & III \\
\hline Sz123B (N) & 16:10:51.31 & $-38: 53: 12.8$ & 2012-04-18 & 56035.2784 & $2 \times 700$ & $2 \times 600$ & $2 \times 350$ & 25 & 70 & 35 & III \\
\hline SST-Lup3-1 ${ }^{\dagger}$ & $16: 11: 59.81$ & $-38: 23: 38.5$ & 2010-04-06 & 55292.3781 & $2 \times 450$ & $2 \times 450$ & $2 \times 450$ & 6 & 35 & 40 & III \\
\hline
\end{tabular}

Notes. ${ }^{(\dagger)}$ Nearby $\left(2{ }^{\prime \prime} 0<d<10{ }^{\prime} 0\right)$ object detected in Spitzer images (see Merín et al. 2008; Comerón 2008). $\left.{ }^{(}\right)$The signal-to-noise ratio (S/N) refers to the central wavelength of each spectrograph arm. 
Table 2. Spectral types, extinction, and physical parameters.

\begin{tabular}{|c|c|c|c|c|c|c|c|c|c|}
\hline Object & SpT & $\begin{array}{l}T_{\text {eff }} \\
{[\mathrm{K}]}\end{array}$ & $\begin{array}{c}A_{V} \\
{[\mathrm{mag}]}\end{array}$ & $\begin{array}{c}d \\
{[\mathrm{pc}]}\end{array}$ & $\begin{array}{c}L_{\star} \\
{\left[L_{\odot}\right]} \\
\end{array}$ & $\begin{array}{c}R_{\star} \\
{\left[R_{\odot}\right]} \\
\end{array}$ & $\begin{array}{c}M_{\star} \\
{\left[M_{\odot}\right]} \\
\end{array}$ & $\begin{array}{r}\text { Age } \\
{[\mathrm{Myr}]}\end{array}$ & Notes \\
\hline Sz66 & M3.0 & 3415 & 1.00 & 150 & $0.200 \pm 0.092$ & $1.29 \pm 0.30$ & $0.45_{-0.15}^{+0.05}$ & 4 & \\
\hline AKC2006-19 & M5.0 & 3125 & 0.00 & 150 & $0.016 \pm 0.008$ & $0.44 \pm 0.10$ & $0.10_{-0.02}^{+0.03}$ & 13 & 1,2 \\
\hline Sz69 & M4.5 & 3197 & 0.00 & 150 & $0.088 \pm 0.041$ & $0.97 \pm 0.22$ & $0.20_{-0.03}^{+0.00}$ & 3 & \\
\hline Sz71 & M1.5 & 3632 & 0.50 & 150 & $0.309 \pm 0.142$ & $1.43 \pm 0.33$ & $0.62_{-0.17}^{+0.02}$ & 4 & \\
\hline Sz72 & M2.0 & 3560 & 0.75 & 150 & $0.252 \pm 0.116$ & $1.29 \pm 0.30$ & $0.45_{-0.00}^{+0.12}$ & 3 & \\
\hline Sz73 & K7 & 4060 & 3.50 & 150 & $0.419 \pm 0.193$ & $1.35 \pm 0.31$ & $1.00_{-0.00}^{+0.00}$ & 9 & \\
\hline Sz74 & M3.5 & 3342 & 1.50 & 150 & $1.043 \pm 0.480$ & $3.13 \pm 0.72$ & $0.50_{-0.10}^{+0.10}$ & 1 & \\
\hline Sz83 & K7 & 4060 & 0.00 & 150 & $1.313 \pm 0.605$ & $2.39 \pm 0.55$ & $1.15_{-0.05}^{+0.25}$ & 2 & \\
\hline Sz84 & M5.0 & 3125 & 0.00 & 150 & $0.122 \pm 0.056$ & $1.21 \pm 0.28$ & $0.17_{-0.02}^{+0.08}$ & 1 & \\
\hline Sz130 & M2.0 & 3560 & 0.00 & 150 & $0.160 \pm 0.074$ & $1.03 \pm 0.24$ & $0.45_{-0.00}^{+0.05}$ & 6 & \\
\hline Sz88A (SW) & M0 & 3850 & 0.25 & 200 & $0.488 \pm 0.225$ & $1.61 \pm 0.37$ & $0.85_{-0.10}^{+0.10}$ & 4 & \\
\hline Sz88B (NE) & M4.5 & 3197 & 0.00 & 200 & $0.118 \pm 0.054$ & $1.12 \pm 0.26$ & $0.20_{-0.03}^{+0.05}$ & 2 & \\
\hline Sz91 & M1 & 3705 & 1.20 & 200 & $0.311 \pm 0.143$ & $1.36 \pm 0.31$ & $0.62_{-0.08}^{+0.13}$ & 4 & \\
\hline Lup713 & M5.5 & 3057 & 0.00 & 200 & $0.020 \pm 0.009$ & $0.52 \pm 0.12$ & $0.08_{-0.00}^{+0.05}$ & 4 & 1 \\
\hline Lup604s & M5.5 & 3057 & 0.00 & 200 & $0.057 \pm 0.026$ & $0.83 \pm 0.19$ & $0.11_{-0.02}^{+0.04}$ & 2 & \\
\hline Sz97 & M4.0 & 3270 & 0.00 & 200 & $0.169 \pm 0.078$ & $1.34 \pm 0.28$ & $0.25_{-0.00}^{+0.05}$ & 2 & \\
\hline Sz99 & M4.0 & 3270 & 0.00 & 200 & $0.074 \pm 0.034$ & $0.89 \pm 0.20$ & $0.17_{-0.00}^{+0.08}$ & 3 & \\
\hline Sz100 & M5.5 & 3057 & 0.00 & 200 & $0.169 \pm 0.078$ & $1.43 \pm 0.33$ & $0.17_{-0.04}^{+0.00}$ & 1 & \\
\hline Sz103 & M4.0 & 3270 & 0.70 & 200 & $0.188 \pm 0.087$ & $1.41 \pm 0.30$ & $0.25_{-0.00}^{+0.05}$ & 1 & \\
\hline Sz104 & M5.0 & 3125 & 0.00 & 200 & $0.102 \pm 0.047$ & $1.11 \pm 0.26$ & $0.15_{-0.02}^{+0.02}$ & 1 & \\
\hline Lup706 & M7.5 & 2795 & 0.00 & 200 & $0.003 \pm 0.001$ & $0.22 \pm 0.05$ & $0.06_{-0.02}^{+0.03}$ & 32 & 1,3 \\
\hline Sz106 & M0.5 & 3777 & 1.00 & 200 & $0.098 \pm 0.045$ & $0.72 \pm 0.17$ & $0.62_{-0.05}^{+0.00}$ & 32 & 3 \\
\hline Par-Lup3-3 & M4.0 & 3270 & 2.20 & 200 & $0.240 \pm 0.110$ & $1.59 \pm 0.37$ & $0.25_{-0.05}^{+0.05}$ & 1 & 1 \\
\hline Par-Lup3-4 & M4.5 & 3197 & 0.00 & 200 & $0.003 \pm 0.001$ & $0.17 \pm 0.04$ & $0.13_{-0.00}^{+0.02}$ & $>50$ & 1,3 \\
\hline Sz110 & M4.0 & 3270 & 0.00 & 200 & $0.276 \pm 0.127$ & $1.61 \pm 0.37$ & $0.35_{-0.05}^{+0.05}$ & 1 & \\
\hline Sz111 & M1 & 3705 & 0.00 & 200 & $0.330 \pm 0.152$ & $1.40 \pm 0.32$ & $0.75_{-0.13}^{+0.05}$ & 6 & \\
\hline Sz112 & M5.0 & 3125 & 0.00 & 200 & $0.191 \pm 0.088$ & $1.52 \pm 0.35$ & $0.25_{-0.08}^{+0.00}$ & 1 & \\
\hline Sz113 & M4.5 & 3197 & 1.00 & 200 & $0.064 \pm 0.030$ & $0.83 \pm 0.19$ & $0.17_{-0.04}^{+0.03}$ & 3 & \\
\hline 2MASS J16085953-3856275 & M8.5 & 2600 & 0.00 & 200 & $0.009 \pm 0.004$ & $0.47 \pm 0.11$ & $0.03_{-0.01}^{+0.01}$ & 1 & 1,2 \\
\hline SSTc2d160901.4-392512 & M4.0 & 3270 & 0.50 & 200 & $0.148 \pm 0.068$ & $1.25 \pm 0.29$ & $0.20_{-0.05}^{+0.10}$ & 1 & \\
\hline Sz114 & M4.8 & 3175 & 0.30 & 200 & $0.312 \pm 0.144$ & $1.82 \pm 0.42$ & $0.30_{-0.10}^{+0.05}$ & 1 & \\
\hline Sz115 & M4.5 & 3197 & 0.50 & 200 & $0.175 \pm 0.080$ & $1.36 \pm 0.31$ & $0.17_{-0.08}^{+0.08}$ & 1 & \\
\hline Lup818s & M6.0 & 2990 & 0.00 & 200 & $0.025 \pm 0.011$ & $0.58 \pm 0.13$ & $0.08_{-0.02}^{+0.02}$ & 3 & 1,2 \\
\hline Sz123A (S) & M1 & 3705 & 1.25 & 200 & $0.203 \pm 0.093$ & $1.10 \pm 0.25$ & $0.60_{-0.03}^{+0.20}$ & 7 & \\
\hline Sz123B (N) & M2.0 & 3560 & 0.00 & 200 & $0.051 \pm 0.024$ & $0.58 \pm 0.13$ & $0.50_{-0.10}^{+0.00}$ & 40 & 3 \\
\hline SST-Lup3-1 & M5.0 & 3125 & 0.00 & 200 & $0.059 \pm 0.027$ & $0.85 \pm 0.19$ & $0.13_{-0.04}^{+0.02}$ & 2 & \\
\hline
\end{tabular}

Notes. 1: Li I $\lambda 670.78 \mathrm{~nm}$ absorption line detected for the first time. The other two targets for which the line is seen for the first time are the class III YSOs Par-Lup3-1 and Par-Lup3-2 (see Manara et al. 2013a); 2: YSO nature confirmed; 3: subluminous YSO. 
Table 3. Accretion properties of Lupus YSOs.

\begin{tabular}{|c|c|c|c|c|c|c|c|c|}
\hline Object & Template & $\mathrm{BJ}_{\mathrm{obs}}$ & $\mathrm{BJ}_{\text {intr }}$ & $\begin{array}{c}\log L_{\text {acc }} \\
{\left[L_{\odot}\right]} \\
\end{array}$ & $\begin{array}{l}\text { Veiling } \\
\text { at } 710 \mathrm{~nm}\end{array}$ & $\begin{array}{r}\log \dot{M}_{\mathrm{acc}} \\
{\left[M_{\odot} \mathrm{yr}^{-1}\right]}\end{array}$ & $L_{\text {all_lines }} / L_{\text {acc }}$ & $\begin{array}{c}\mathrm{WH} \alpha(10 \%) \\
{\left[\mathrm{km} \mathrm{s}^{-1}\right]}\end{array}$ \\
\hline Sz66 & SO797 & 1.50 & 1.90 & -1.8 & 0.45 & -8.73 & 0.09 & 460 \\
\hline AKC2006-19 & SO641 & 0.60 & 14.67 & -4.1 & 0.04 & -10.85 & 0.16 & 228 \\
\hline Sz69 & SO797 & 2.83 & 4.50 & -2.8 & 0.18 & -9.50 & 0.55 & 403 \\
\hline Sz71 & TWA15A & 1.19 & 2.65 & -2.2 & 0.08 & -9.23 & 0.24 & 350 \\
\hline Sz72 & TWA9B & 2.73 & 3.70 & -1.8 & 0.23 & -8.73 & 0.28 & 455 \\
\hline Sz73 & SO879 & 1.05 & 2.08 & -1.0 & 0.22 & -8.26 & 0.05 & 504 \\
\hline Sz74 & TWA15A & 0.90 & 1.46 & -1.5 & 0.10 & -8.09 & 0.07 & 401 \\
\hline Sz83 & SO879 & 2.28 & 2.27 & -0.3 & 1.88 & -7.37 & 0.14 & 604 \\
\hline Sz84 & SO641 & 1.44 & 2.65 & -2.7 & 0.10 & -9.24 & 0.28 & 456 \\
\hline Sz130 & TWA2A & 1.50 & 2.43 & -2.2 & 0.15 & -9.23 & 0.20 & 266 \\
\hline Sz88A (SW) & TWA25 & 2.08 & 3.03 & -1.2 & 0.32 & -8.31 & 0.18 & 597 \\
\hline Sz88B (NE) & SO797 & 0.87 & 3.34 & -3.1 & 0.04 & -9.74 & 0.18 & 405 \\
\hline Sz91 & TWA13A & 1.12 & 2.14 & -1.8 & 0.12 & -8.85 & 0.22 & 374 \\
\hline Lup713 & Par-Lup3-2 & 3.50 & 6.50 & -3.5 & 0.12 & -10.08 & 0.54 & 378 \\
\hline Lup604s & SO925 & 0.50 & 8.50 & -3.7 & 0.05 & -10.21 & 0.13 & 264 \\
\hline Sz97 & Sz94 & 1.00 & 3.70 & -2.9 & 0.03 & -9.56 & 0.38 & 452 \\
\hline Sz99 & TWA9B & 1.90 & 2.65 & -2.6 & 0.26 & -9.27 & 0.19 & 373 \\
\hline Sz100 & SO641 & 0.87 & 2.16 & -3.0 & 0.09 & -9.47 & 0.31 & 251 \\
\hline Sz103 & Sz94 & 0.74 & 1.87 & -2.4 & 0.14 & -9.04 & 0.09 & 426 \\
\hline Sz104 & SO641 & 0.65 & 1.90 & -3.2 & 0.06 & -9.72 & 0.23 & 201 \\
\hline Lup706 & TWA26 & 2.40 & 13.50 & -4.8 & 0.11 & -11.63 & 0.51 & 328 \\
\hline Sz106 & TWA25 & 0.97 & 1.87 & -2.5 & 0.14 & -9.83 & 0.06 & 459 \\
\hline Par-Lup3-3 & TWA15A & 0.50 & 1.10 & -2.9 & 0.01 & -9.49 & 0.19 & 240 \\
\hline Par-Lup3-4 & SO641 & 2.00 & 3.00 & -4.1 & 0.25 & -11.37 & 0.46 & 393 \\
\hline Sz110 & Sz94 & 1.43 & 2.14 & -2.0 & 0.26 & -8.73 & 0.20 & 498 \\
\hline Sz111 & TWA13A & 1.36 & 9.60 & -2.2 & 0.04 & -9.32 & 0.41 & 455 \\
\hline Sz112 & SO641 & 0.40 & 1.40 & -3.2 & 0.03 & -9.81 & 0.15 & 160 \\
\hline Sz113 & SO797 & 1.86 & 2.29 & -2.1 & 0.56 & -8.80 & 0.20 & 392 \\
\hline 2MASS J16085953-3856275 & TWA26 & 3.69 & 15.00 & -4.6 & 0.08 & -10.80 & 0.39 & 147 \\
\hline SSTc2d160901.4-392512 & $\mathrm{Sz} 94$ & 0.84 & 3.85 & -3.0 & 0.04 & -9.59 & 0.35 & 447 \\
\hline Sz114 & Sz94 & 0.67 & 2.07 & -2.5 & 0.05 & -9.11 & 0.26 & 222 \\
\hline Sz115 & SO797 & 0.53 & 1.00 & -2.7 & 0.10 & -9.19 & 0.07 & 338 \\
\hline Lup818s & SO925 & 1.06 & 4.00 & -4.1 & 0.08 & -10.63 & 0.48 & 200 \\
\hline Sz123A (S) & TWA2A & 1.71 & 2.71 & -1.8 & 0.22 & -8.93 & 0.30 & 487 \\
\hline Sz123B (N) & TWA15B & 1.36 & 2.45 & -2.7 & 0.09 & -10.03 & 0.36 & 519 \\
\hline SST-Lup3-1 & SO641 & 1.23 & 6.00 & -3.6 & 0.03 & -10.17 & 0.48 & 254 \\
\hline
\end{tabular}


Table 4. Results of the $L_{\text {acc }}-L_{\text {line }}$ linear fits.

\begin{tabular}{|c|c|c|c|c|c|c|c|}
\hline Diagnostic & $\begin{array}{r}\lambda \\
{[\mathrm{nm}]}\end{array}$ & $a( \pm$ err $)$ & $b( \pm$ err $)$ & $N_{\text {points }}^{\dagger}$ & $\sigma^{\star}$ & $\begin{array}{c}\text { No. upper } \\
\text { limits }\end{array}$ & Comment \\
\hline $\mathrm{H} 3(\mathrm{H} \alpha)$ & 656.2800 & $1.12(0.07)$ & $1.50(0.26)$ & $36+6$ & 0.36 & 0 & \\
\hline $\mathrm{H} 4(\mathrm{H} \beta)$ & 486.1325 & $1.11(0.05)$ & $2.31(0.23)$ & $36+6$ & 0.27 & 0 & $\bullet$ \\
\hline $\mathrm{H} 5(\mathrm{H} \gamma)$ & 434.0464 & $1.09(0.05)$ & $2.50(0.25)$ & $36+6$ & 0.27 & 0 & $\bullet$ \\
\hline H6 $(\mathrm{H} \delta)$ & 410.1734 & $1.06(0.06)$ & $2.50(0.28)$ & 36 & 0.30 & 0 & $\bullet$ \\
\hline $\mathrm{H} 7(\mathrm{H} \epsilon)$ & 397.0072 & $1.07(0.06)$ & $2.64(0.29)$ & 36 & 0.30 & 0 & 1 \\
\hline H8 & 388.9049 & $1.04(0.06)$ & $2.55(0.29)$ & 36 & 0.30 & 0 & $\bullet$ \\
\hline H9 & 383.5384 & $1.01(0.05)$ & $2.53(0.27)$ & 36 & 0.29 & 0 & • \\
\hline H10 & 379.7898 & $1.00(0.05)$ & $2.58(0.27)$ & 35 & 0.29 & 1 & $\bullet$ \\
\hline H11 & 377.0630 & $1.02(0.05)$ & $2.74(0.25)$ & $35+6$ & 0.27 & 1 & $\bullet$ \\
\hline H12 & 375.0151 & $0.99(0.05)$ & $2.73(0.25)$ & 35 & 0.26 & 1 & • \\
\hline H13 & 373.4368 & $1.00(0.05)$ & $2.85(0.25)$ & 34 & 0.25 & 2 & $\bullet$ \\
\hline H14 & 372.1938 & $1.02(0.06)$ & $3.09(0.31)$ & 31 & 0.25 & 5 & $\bullet$ \\
\hline H15 & 371.1977 & $1.02(0.06)$ & $3.13(0.31)$ & 31 & 0.25 & 5 & $\bullet$ \\
\hline $\mathrm{Pa} 5(\mathrm{~Pa} \beta)$ & 1281.8070 & $1.04(0.08)$ & $2.45(0.39)$ & $29+6$ & 0.38 & 7 & $\bullet$ \\
\hline Pa6 (Pay) & 1093.8086 & $1.18(0.06)$ & $3.17(0.31)$ & $33+6$ & 0.29 & 3 & - \\
\hline $\mathrm{Pa} 7(\mathrm{~Pa} \delta)$ & 1004.9368 & $1.18(0.10)$ & $3.33(0.47)$ & 25 & 0.36 & 9 & $\bullet$ \\
\hline $\mathrm{Pa} 8$ & 954.5969 & $1.11(0.12)$ & $3.19(0.58)$ & 17 & 0.39 & 16 & \\
\hline $\mathrm{Pa} 9$ & 922.9014 & $1.13(0.09)$ & $3.40(0.47)$ & 27 & 0.35 & 7 & \\
\hline Pa10 & 901.4909 & $1.03(0.09)$ & $2.99(0.49)$ & 26 & 0.39 & 8 & \\
\hline $\operatorname{Br} 7(\operatorname{Br} \gamma)$ & 2166.1210 & $1.16(0.07)$ & $3.60(0.38)$ & 19 & 0.28 & 14 & $\bullet$ \\
\hline He I & 402.6191 & $1.04(0.06)$ & $3.62(0.37)$ & 31 & 0.27 & 5 & $\bullet$ \\
\hline He I & 447.1480 & $1.05(0.06)$ & $3.45(0.35)$ & 33 & 0.30 & 3 & $\bullet$ \\
\hline He I & 471.3146 & $1.04(0.11)$ & $4.25(0.69)$ & 16 & 0.29 & 12 & $\bullet$ \\
\hline He IFe I & 492.1931 & $0.90(0.06)$ & $2.65(0.36)$ & 32 & 0.31 & 4 & 2 \\
\hline $\mathrm{He} \mathrm{I}$ & 501.5678 & $0.98(0.06)$ & $3.36(0.38)$ & 30 & 0.27 & 4 & $\bullet$ \\
\hline He I & 587.5621 & $1.13(0.06)$ & $3.51(0.30)$ & $36+6$ & 0.28 & 0 & - \\
\hline He I & 667.8151 & $1.16(0.08)$ & $4.12(0.45)$ & 36 & 0.36 & 0 & $\bullet$ \\
\hline He I & 706.5190 & $1.14(0.07)$ & $4.16(0.39)$ & 36 & 0.32 & 0 & $\bullet$ \\
\hline He I & 1082.9091 & $1.11(0.12)$ & $2.62(0.57)$ & 30 & 0.44 & 4 & 3 \\
\hline He II & 468.5804 & $1.10(0.09)$ & $4.21(0.57)$ & 28 & 0.36 & 2 & \\
\hline Ca II (K) & 393.3660 & $0.96(0.05)$ & $2.06(0.27)$ & 36 & 0.31 & 0 & $\bullet$ \\
\hline Ca II (H) & 396.8470 & $1.02(0.05)$ & $2.37(0.23)$ & 36 & 0.25 & 0 & 4 \\
\hline Ca II & 849.8020 & $0.95(0.07)$ & $2.18(0.38)$ & 34 & 0.41 & 2 & \\
\hline Ca II & 854.2090 & $0.95(0.08)$ & $2.13(0.42)$ & 32 & 0.44 & 2 & \\
\hline Ca II & 866.2140 & $0.95(0.09)$ & $2.20(0.43)$ & 29 & 0.43 & 4 & \\
\hline $\mathrm{NaI}$ & 588.995 & $0.93(0.06)$ & $2.56(0.32)$ & 36 & 0.33 & 0 & \\
\hline $\mathrm{NaI}$ & 589.592 & $0.90(0.06)$ & $2.56(0.37)$ & 36 & 0.38 & 0 & \\
\hline O I & 777.3055 & $1.16(0.09)$ & $3.91(0.51)$ & 14 & 0.36 & 6 & 5 \\
\hline OI & 844.6360 & $1.06(0.18)$ & $3.06(0.90)$ & 18 & 0.61 & 3 & \\
\hline
\end{tabular}

Notes. The relations are of the form $\log \left(L_{\text {acc }} / L_{\odot}\right)=a \cdot \log \left(L_{\text {line }} / L_{\odot}\right)+b{ }^{(\dagger)}$ Number of points for the fit. The fits in which the six YSOs in $\sigma$-Ori (Rigliaco et al. 2012) were included are indicated with "+6". Although measurements of the Ca II IRT are also available in Rigliaco et al. (2012), we did not include them because those measurements were not corrected for the photospheric contribution. The number of points for Lupus is the number of YSOs in which the corresponding line was detected. ${ }^{(\star)}$ Standard deviation from linear fit. Comments in last column: (1) partially blended with $\mathrm{Ca}$ II H; (2) He I + Fe I blend; (3) this line is also produced in winds/outflows (Edwards et al. 2006); the relationship must be used with caution; (4) partially blended with $\mathrm{H} \epsilon$; (5) O I $\lambda \lambda$ 777.194, $777.417 \mathrm{~nm}$ doublet. •: Suggested relations for deriving $L_{\text {acc }}$ from the line luminosity. 


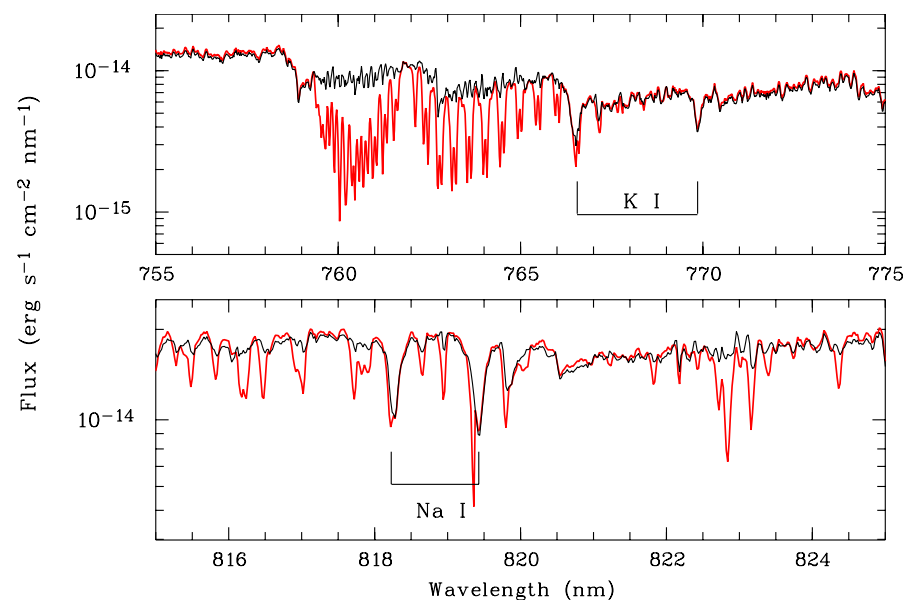

Fig. A.1. Example of telluric-corrected spectrum (black lines) of the YSO Lup713 in the range of the K I $\lambda \lambda 766.49,769.90 \mathrm{~nm}$ (upper panel) and the Na I $\lambda \lambda 818.33,819.48 \mathrm{~nm}$ (lower panel) doublets. The uncorrected spectra are overlaid with red lines.

\section{Appendix A: Correction for telluric bands}

The flux-calibrated one-dimensional spectra resulting from the $\mathrm{X}$-shooter pipeline or our MIDAS procedure are not corrected for the contribution of telluric bands. Therefore, the telluric standards were used to perform the correction using the IRAF task "telluric". The procedure basically consists of dividing the target spectrum by the telluric spectrum multiplied by an appropriate scaling factor. This factor depends on the ratio of the airmass of the target and the telluric standard. Since the targets and their assigned telluric standards were observed at very similar airmasses in most cases, this factor is typically close to one. The procedure was applied independently in the VIS and NIR spectra, in a different way.

\section{A.1. Telluric correction in the VIS spectra}

The correction was made directly on the one-dimensional fluxcalibrated VIS spectra of the targets using the assigned tellurics. However, to avoid introducing of the SED of the telluric on the flux-calibrated spectra, the tellurics were first normalised to their continuum and their hydrogen lines and other stellar lines were removed by fitting combinations of Gaussian, Lorentzian, and Voigt functions. The resulting normalised telluric spectra, free of photospheric lines, were then used as input in the IRAF task "telluric". In Fig. A.1 examples of the telluric correction in the spectral range of the KI $\lambda \lambda 766.49,769.90 \mathrm{~nm}$ and the $\mathrm{Na}$ I $\lambda \lambda 818.33,819.481 \mathrm{~nm}$ doublets are shown.

\section{A.2. Telluric correction in the NIR spectra}

For the NIR spectra, a pseudo-response function was first derived by dividing the not flux-calibrated telluric by a black-body of the same effective temperature as the telluric. This pseudoresponse function was also cleaned for photospheric lines in the same way as in the VIS. The resulting pseudo-response function, free of stellar lines and containing the telluric bands, was then used as input in the IRAF task "telluric". Note that the target spectrum to be used as input of the IRAF task was the not flux-calibrated one-dimensional spectrum. In this way, the telluric correction and the correction for the response function were made simultaneously in the NIR. Although the shape of the

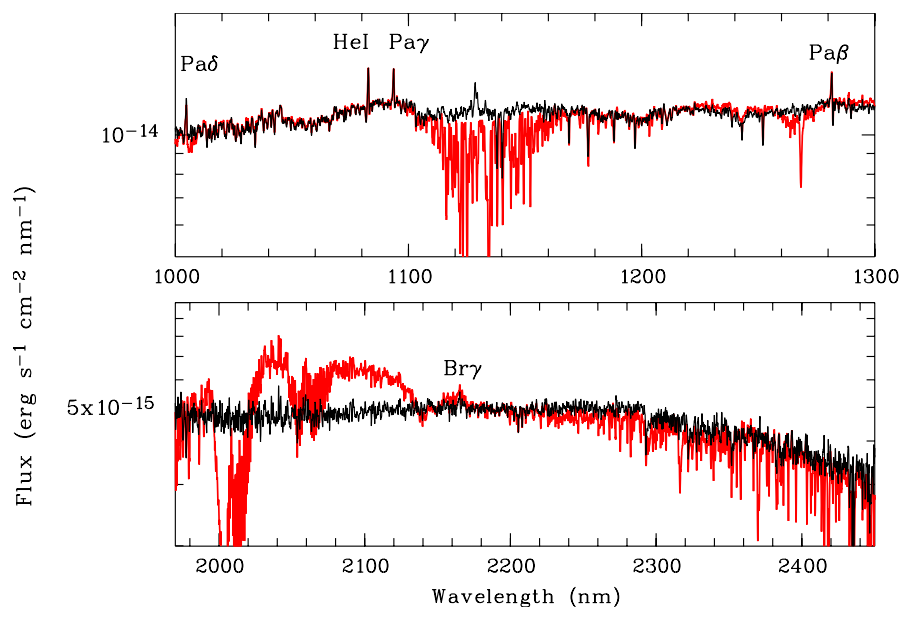

Fig. A.2. Examples of the telluric-corrected spectrum of Lup713 in two spectral ranges of the NIR arm. The uncorrected spectra are overlaid with red lines in both panels.

resulting spectrum after this procedure is correct, the flux calibration is made only relative to the pseudo-response function. Therefore, a factor was applied to bring the NIR spectrum to the absolute flux scale. This factor was estimated using the not telluric-corrected, but flux-calibrated spectra resulting from the $\mathrm{X}$-shooter pipeline and our MIDAS procedure. In Fig. A.2 examples of the telluric correction of the YSO Lup713 are shown in two spectral ranges of the NIR arm. Note that in the range 2000-2140 nm the pipeline yields a bump with respect to the telluric corrected spectrum. This defect in the latter was corrected for because the pseudo-response function contains this bump as well.

\section{Appendix B: Impact of veiling on spectral typing and extinction}

\section{B.1. Veiling and spectral indices}

Strong veiling may influence the spectral indices used to classify the M-type YSOs. To investigate the impact of veiling on the spectral indices, we proceeded as follows. A constant veiling was artificially added to the spectra of the class III templates in the spectral range between $740 \mathrm{~nm}$ and $860 \mathrm{~nm}$, where the indices are computed. Then, the new indices and spectral type of the "veiled" spectrum were derived. The effect of veiling is to yield systematically earlier types, but in that spectral range the veiling is expected to be stronger than one to induce a difference of about one spectral subclass. As seen in Sect. 4.1.1, the strongest veiling, at $710 \mathrm{~nm}$, among the M-type YSOs is lower than 0.6 and corresponds to Sz 113. For this object we estimate that veiling would introduce an uncertainty of 0.4 subclass in spectral type. For the other YSOs the excess emission in the Paschen continuum is lower (see Table 3). For all the M-type YSOs veiling is estimated to influence the spectral type classification by less than 0.3 spectral subclasses.

\section{B.2. Veiling and extinction}

The effect of strong veiling is to make YSOs objects intrinsically bluer than the templates we used to derive extinction. Thus, our method may underestimate extinction if veiling is very strong. To investigate the extent of this, we proceeded as follows. The observed spectrum of a given $\mathrm{YSO}, F_{\mathrm{YSO}}(\lambda)$, is 
the sum of the flux of the object, $F_{*}(\lambda)$, plus the flux of the continuum excess emission, $F_{\text {cont }}(\lambda)$, extincted with an extinction law, $A_{\lambda} / A_{V}$. Accordingly, the flux we observe is $F_{\mathrm{YsO}}(\lambda)=$

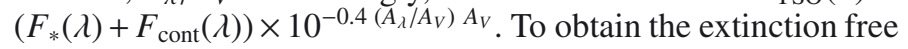
of veiling effects, we must apply our methods, not to $F_{\mathrm{YSO}}(\lambda)$, but to the de-veiled spectrum:

$$
F_{\mathrm{YSO}}(\lambda)-F_{\text {cont }}(\lambda) \times 10^{-0.4\left(A_{\lambda} / A_{V}\right) A_{V}}
$$

We can assume that the continuum flux is the model continuum excess emission derived in Sect. 4.1.1, hence from Eq. (B.1) we can compute a de-veiled spectrum, provided we know $A_{V}$. Since we do not know $A_{V}$ in advance, we proceeded in a two-step fitting procedure. As first guess of $A_{V}$ we used the value derived from our template fitting procedure applied to $F_{\mathrm{YsO}}(\lambda)$, and computed a de-veiled spectrum using Eq. (B.1). A new $A_{V}$ was then derived from our fitting procedure applied to the first de-veiled spectrum. This spectrum was used to compute a new $A_{V}$ value and to redden the $F_{\text {cont }}(\lambda)$ to be used again to calculate a second de-veiled spectrum, and so on until a $A_{V}$ value was found that leads to the best match with the template.

In practice, we used $\mathrm{Sz} 113$ as a test case, the most strongly veiled M-Type YSO in our sample. The extinction we derived as first guess is $1 \mathrm{mag}$. Thus, the model continuum excess emission derived in Sect. 4.1.1 was reddened at extinctions between $1 \mathrm{mag}$ and $2 \mathrm{mag}$ in steps of $0.25 \mathrm{mag}$. The results were then subtracted from the observed spectrum of $\mathrm{Sz} 113$. The several de-veiled spectra were then processed with our fitting procedure. The best compromise between reddened model continuum and de-reddened de-veiled spectra was found at $A_{V}=1.5 \mathrm{mag}$. We conclude that for our most strongly veiled M-Type YSO extinction is underestimated by less than $0.5 \mathrm{mag}$. 


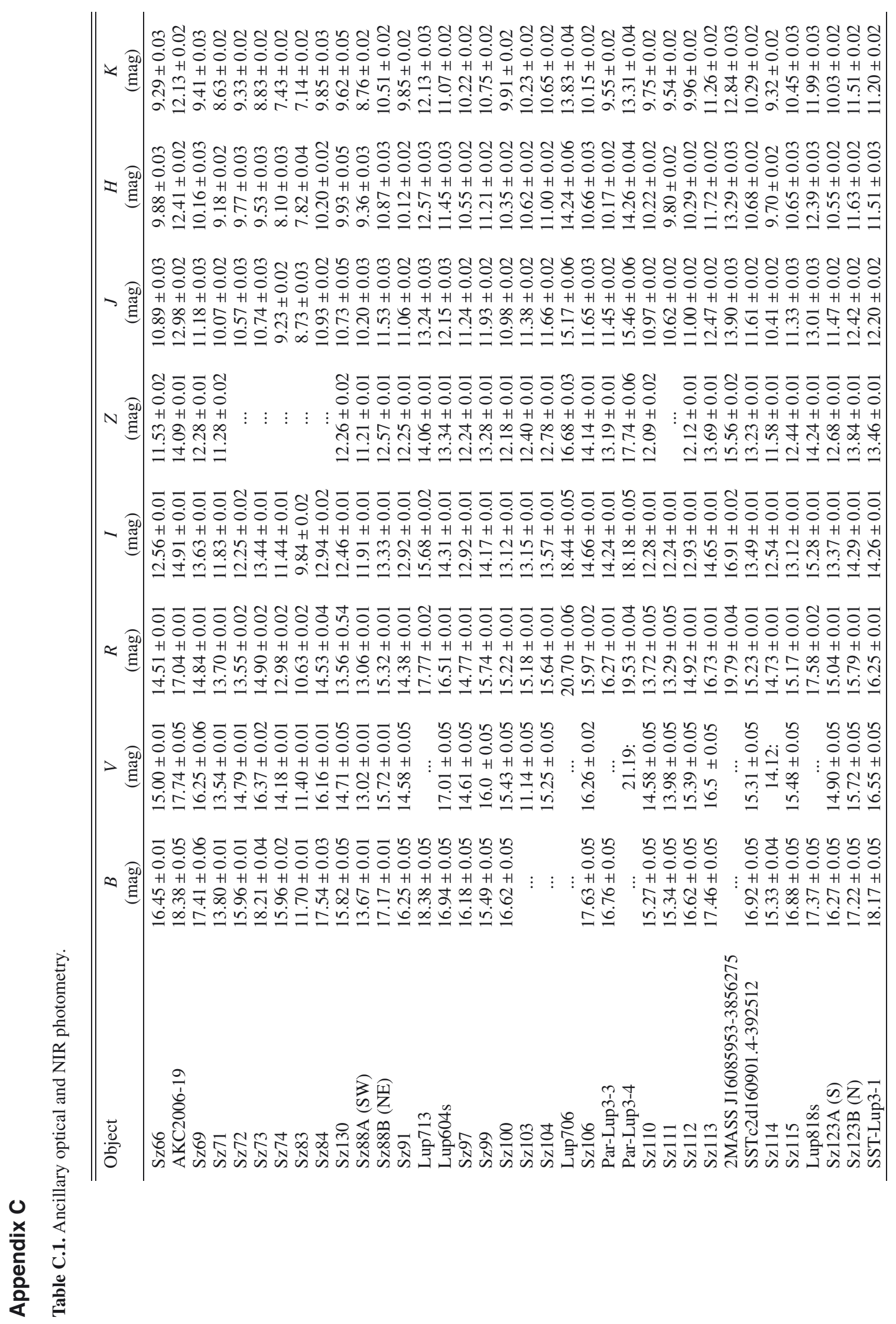


A\&A 561, A2 (2014)

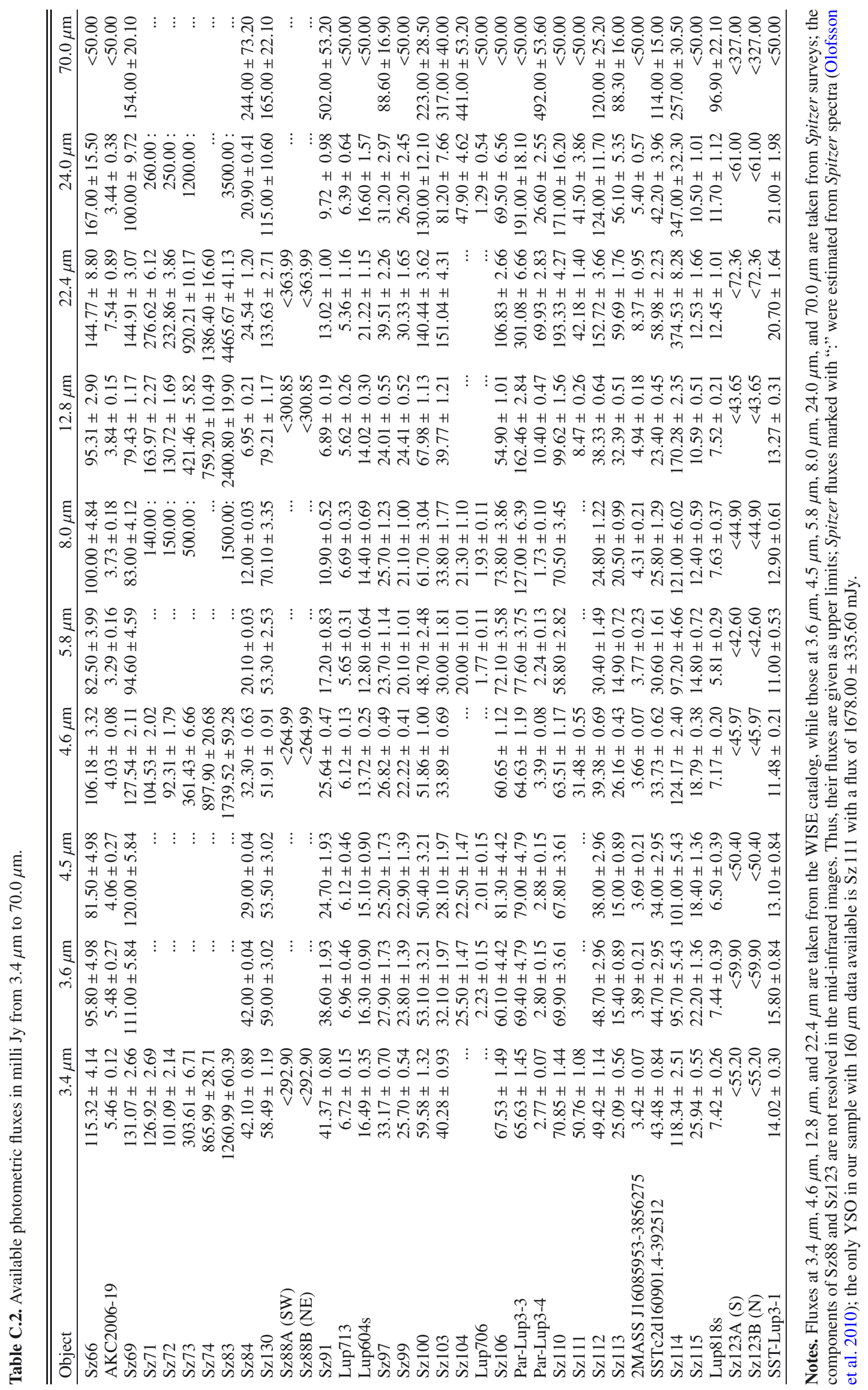


J. M. Alcalá et al.: Accretion in Lupus YSOs
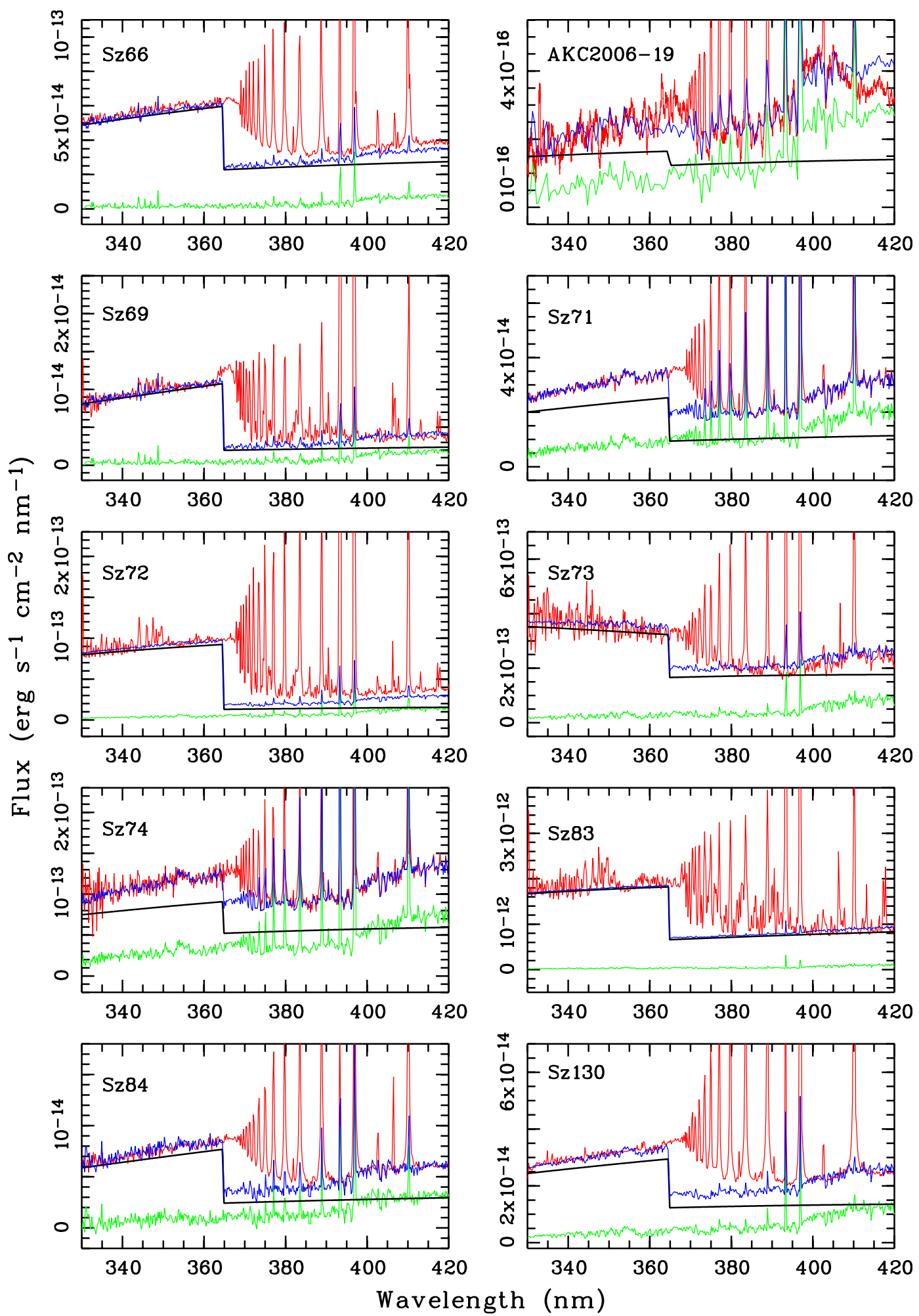

Fig. C.1. Extinction-corrected spectra (red) fitted with a combination of a photospheric template (green) and the synthetic continuum spectrum from a hydrogen slab (black). The total fit is represented with the blue line. 

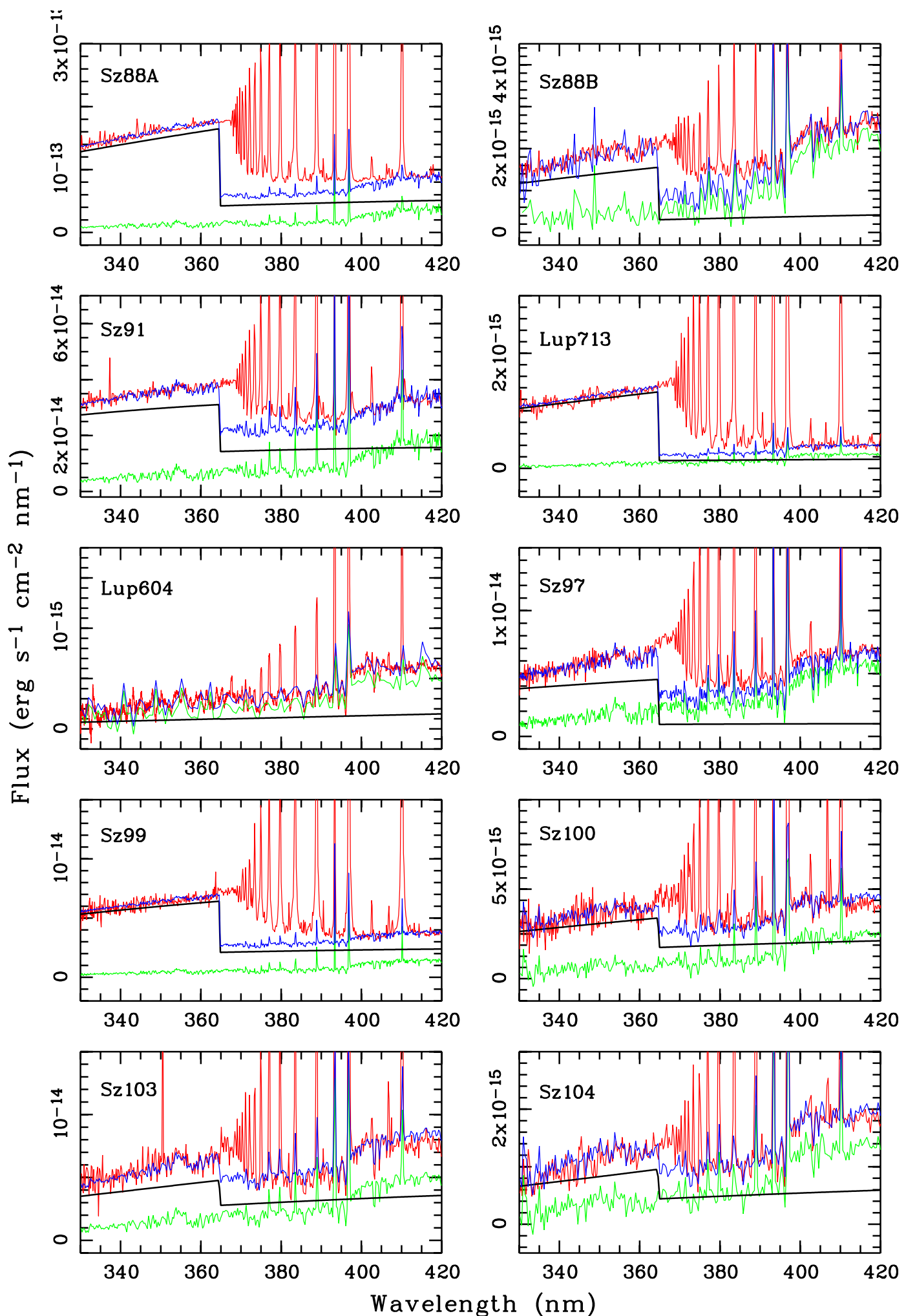

Fig. C.2. Extinction-corrected spectra (red) fitted with a combination of a photospheric template (green) and the synthetic continuum spectrum from a hydrogen slab (black). The total fit is represented with the blue line. 
J. M. Alcalá et al.: Accretion in Lupus YSOs
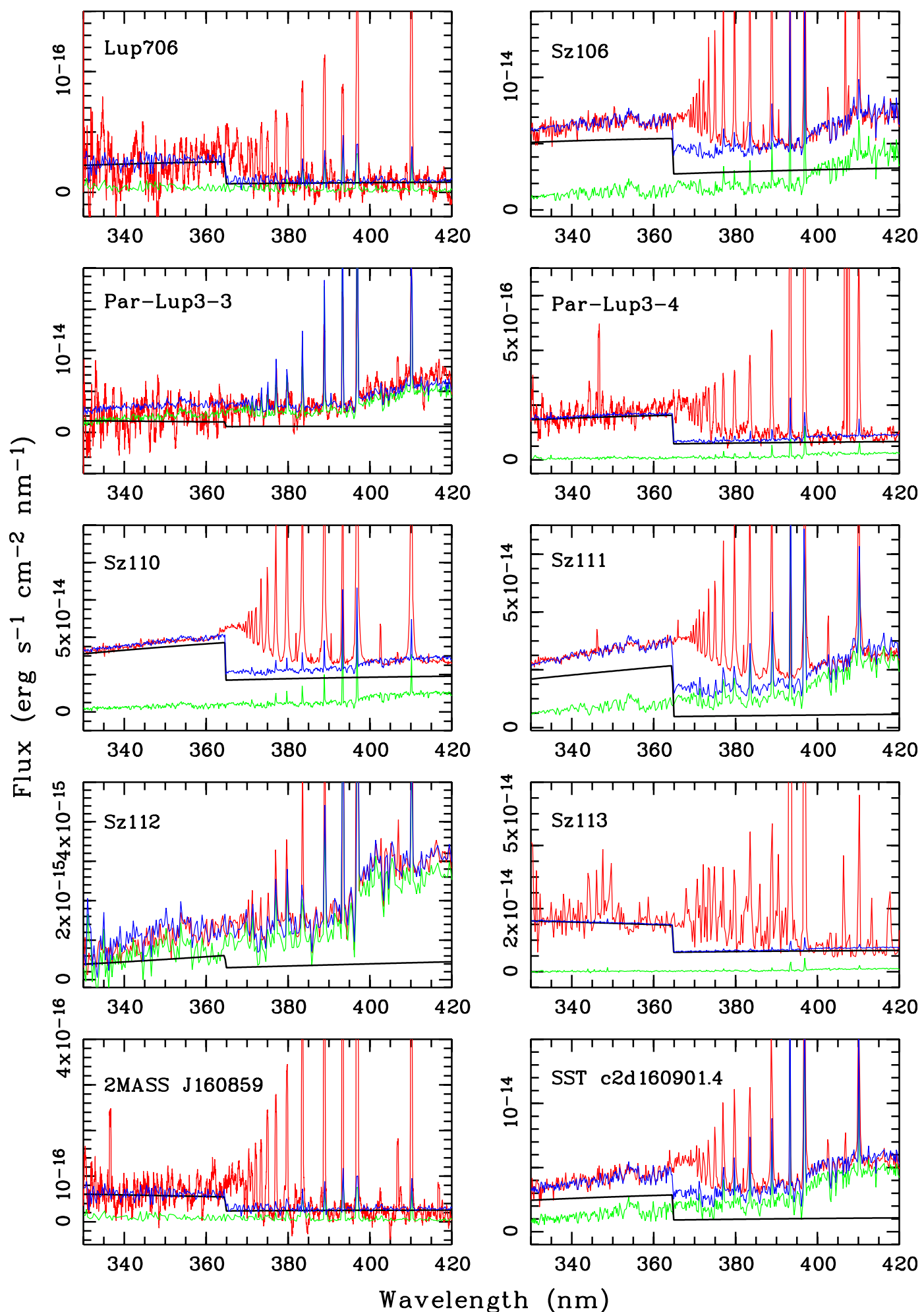

Fig. C.3. Extinction-corrected spectra (red) fitted with a combination of a photospheric template (green) and the synthetic continuum spectrum from a hydrogen slab (black). The total fit is represented with the blue line. 

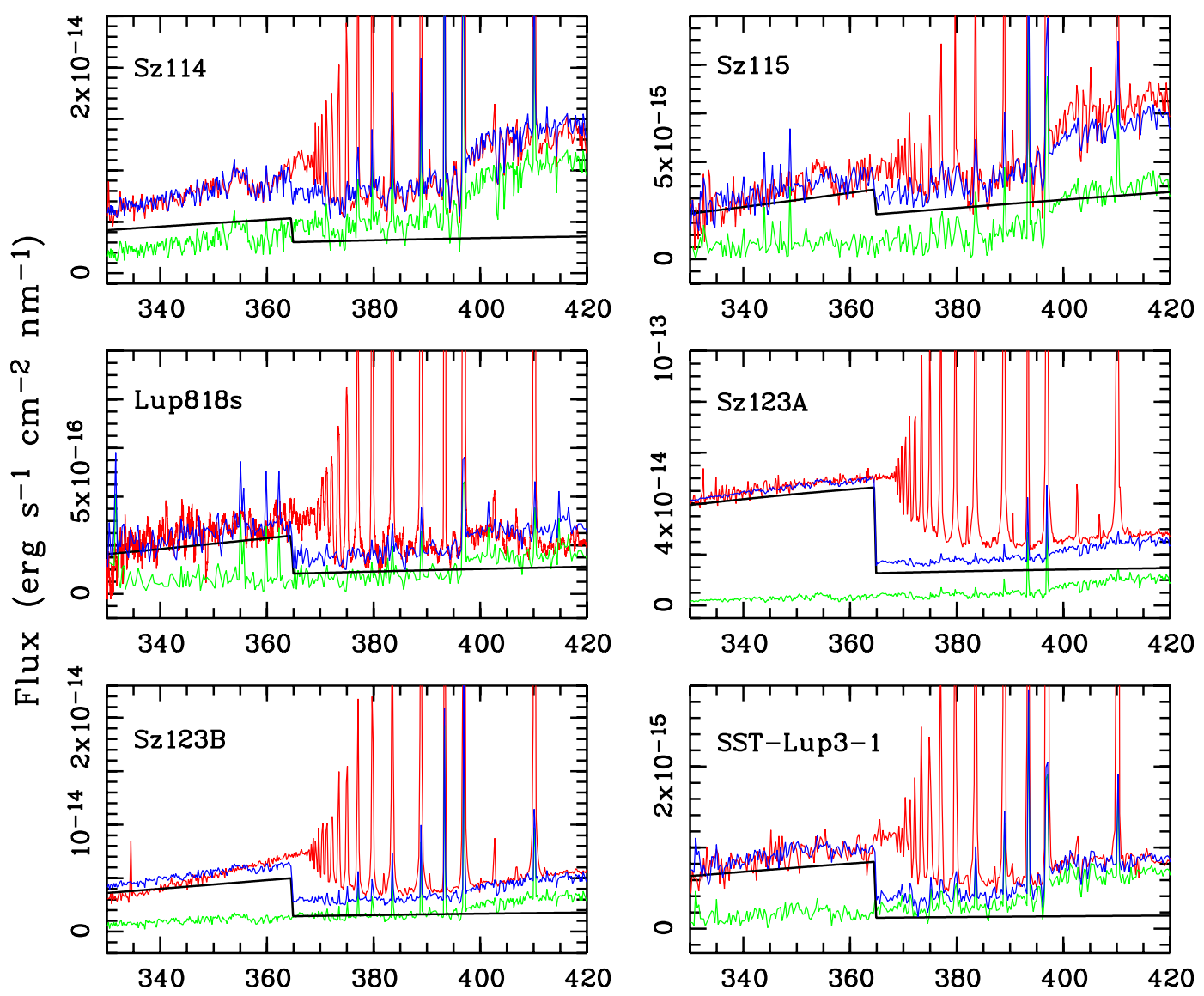

Wavelength $(\mathrm{nm})$

Fig. C.4. Extinction-corrected spectra (red) fitted with a combination of a photospheric template (green) and the synthetic continuum spectrum from a hydrogen slab (black). The total fit is represented with the blue line. 


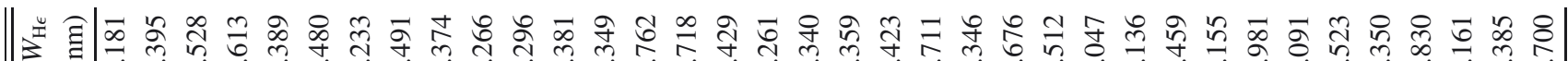

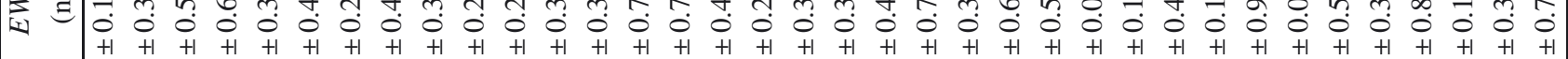

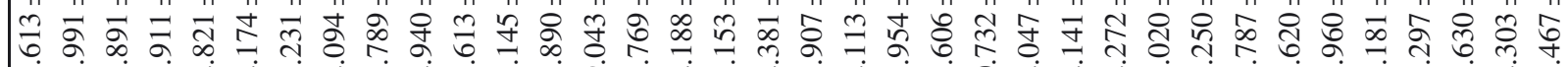

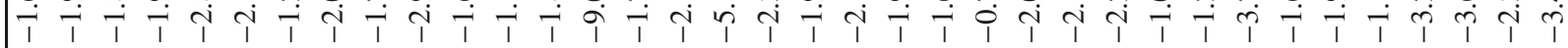

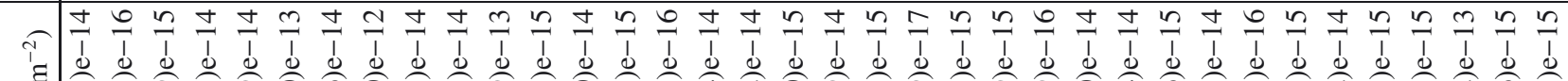
ঊ 等

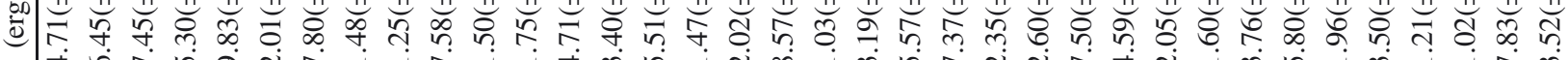

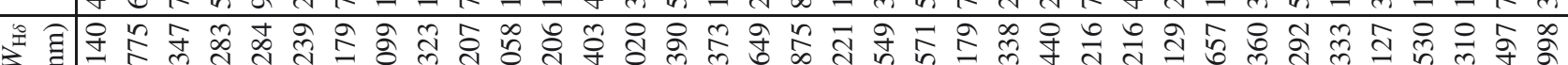

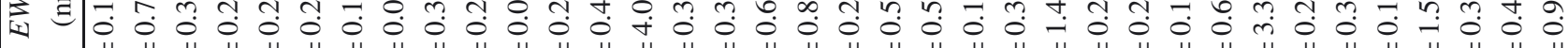

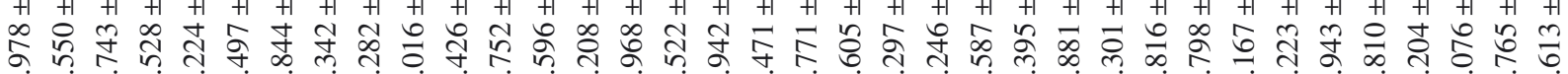
i

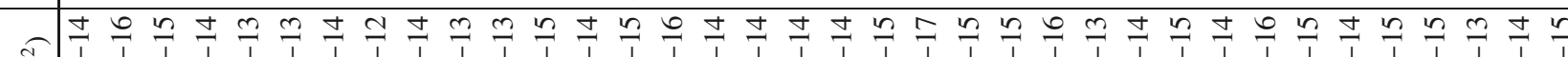

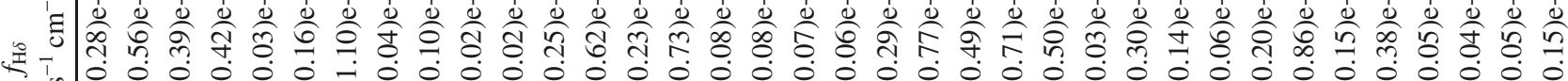

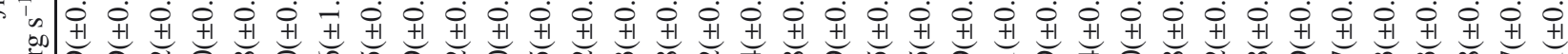

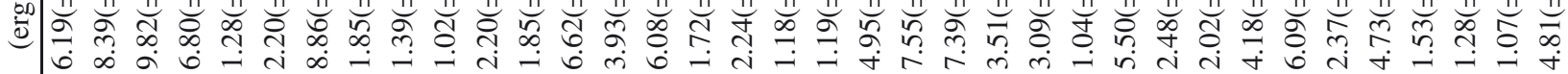

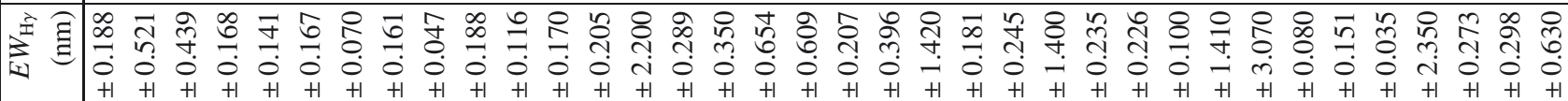

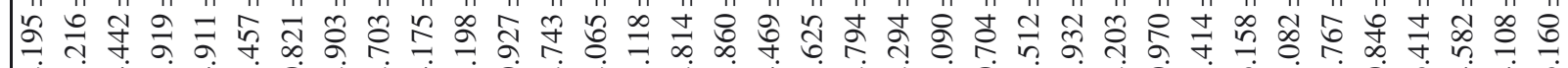
i

ง

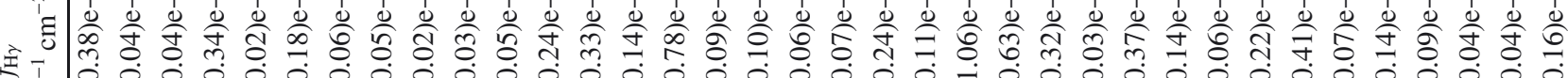

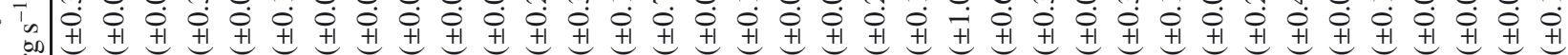

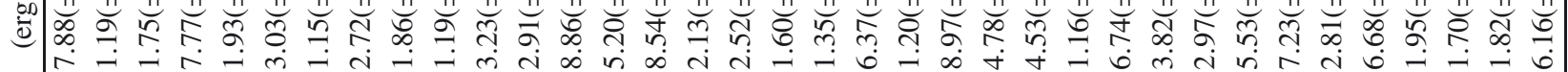

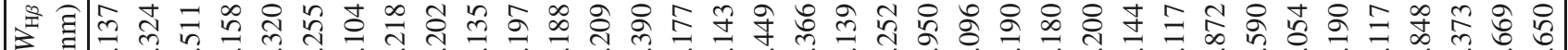

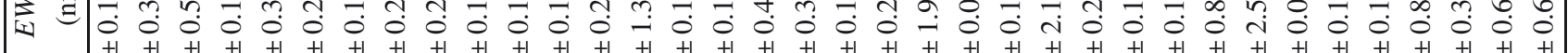

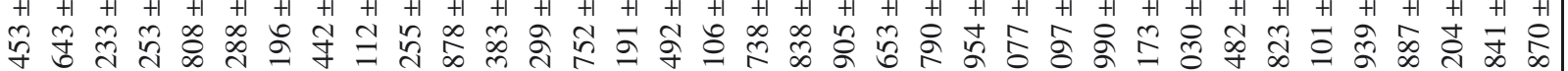
i

e

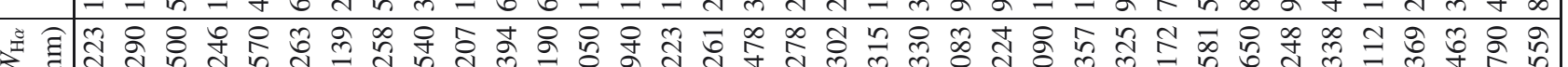
可

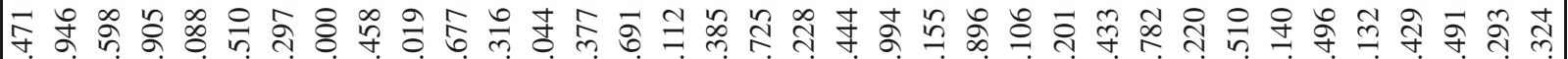
i i

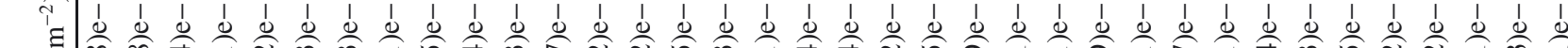

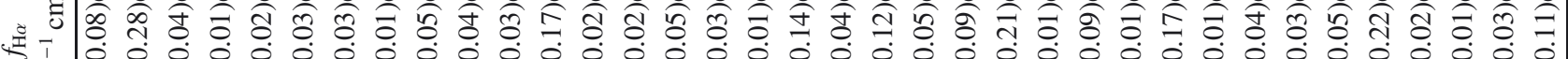

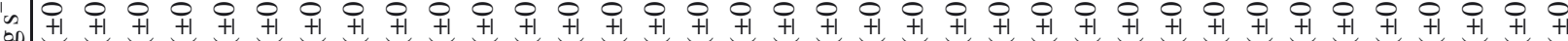
党|

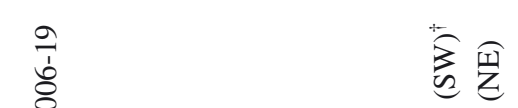

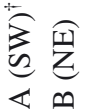

\& 0 : 死方 


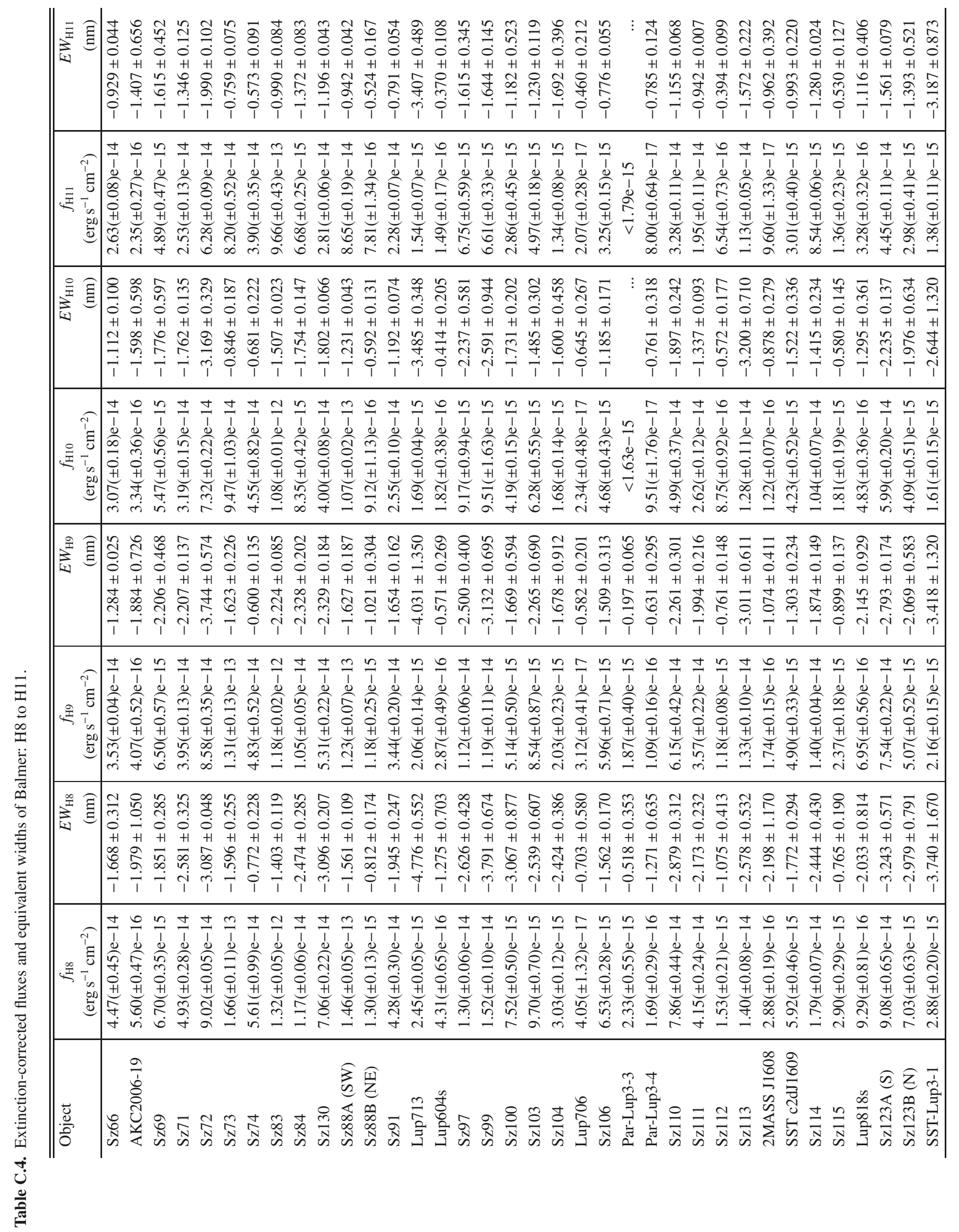

A2, page 30 of 43 


\begin{tabular}{|c|c|c|}
\hline & 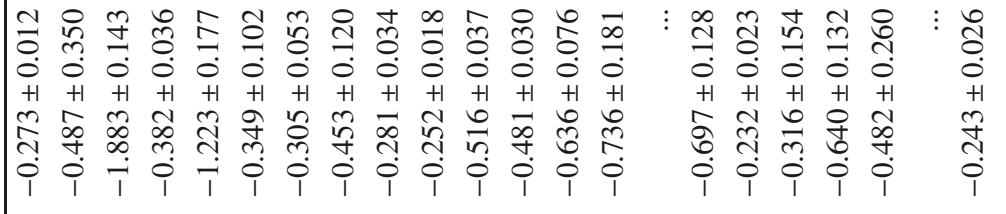 & 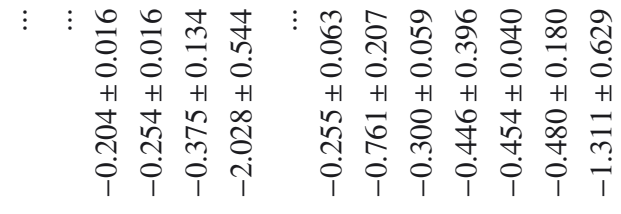 \\
\hline & 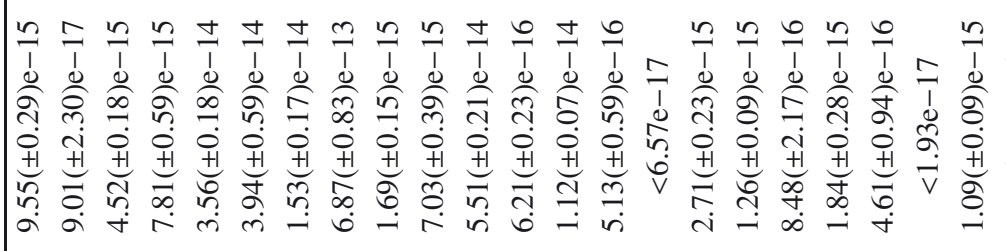 & 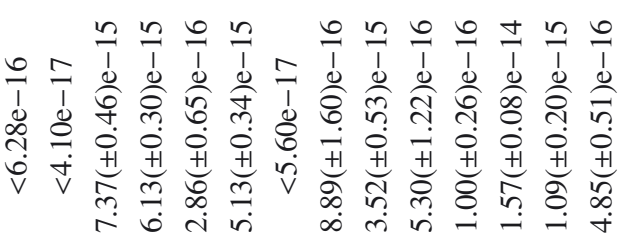 \\
\hline & 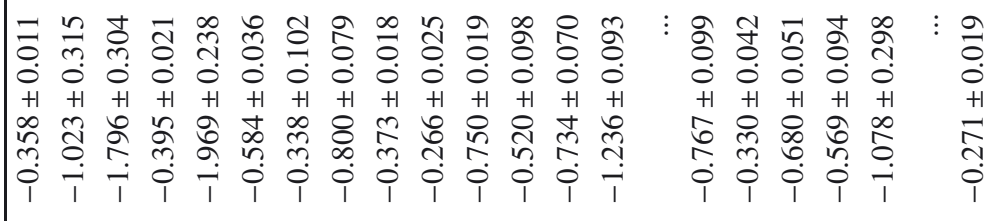 & 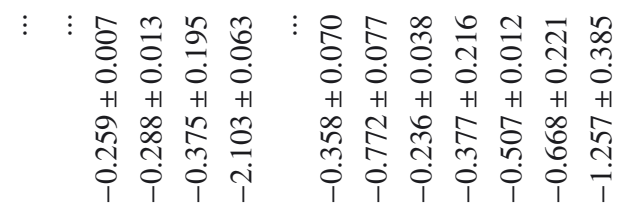 \\
\hline & 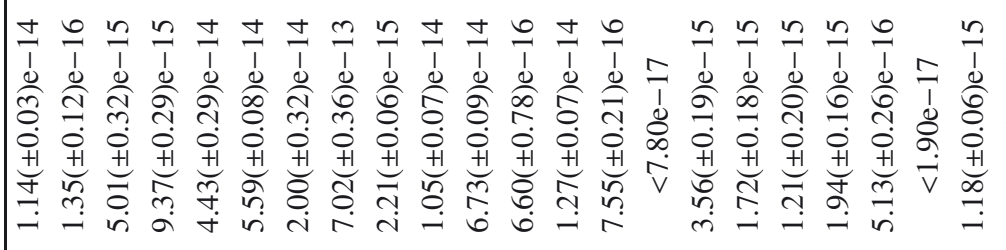 & 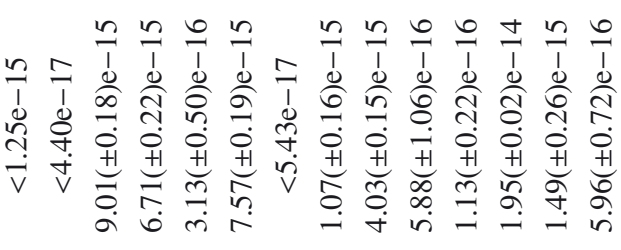 \\
\hline & 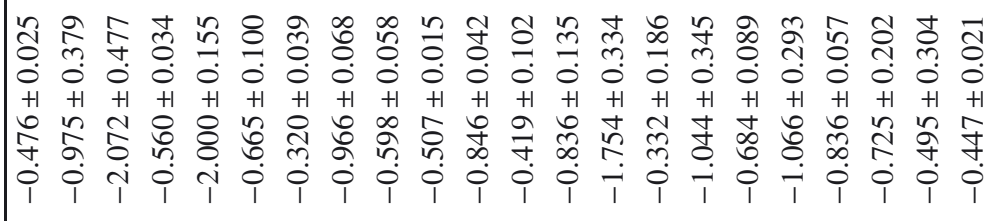 & 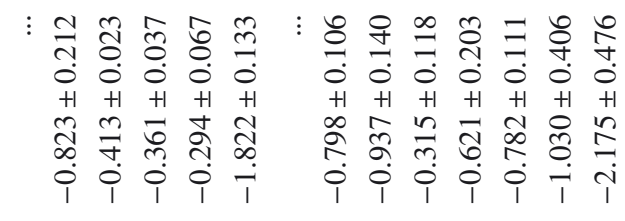 \\
\hline & 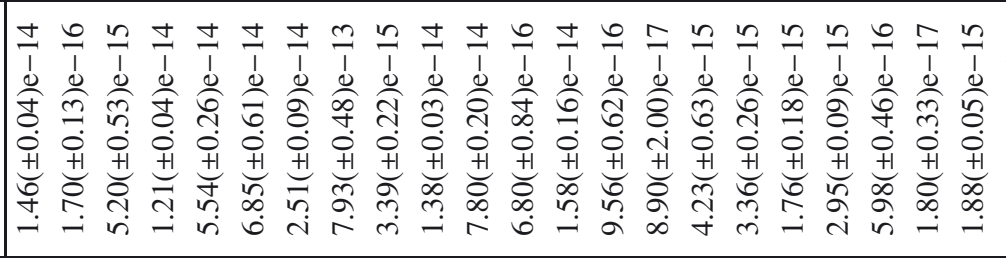 & 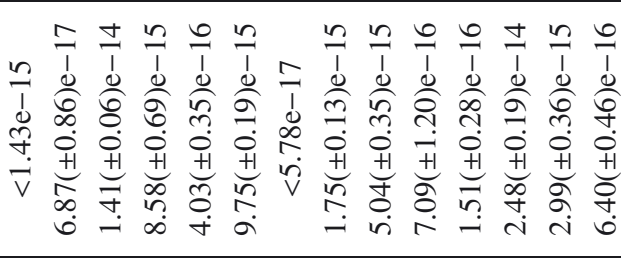 \\
\hline & 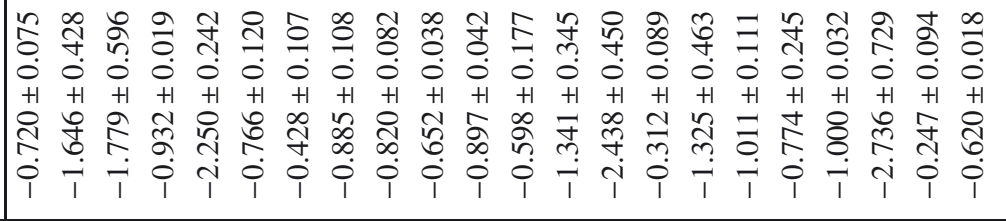 & 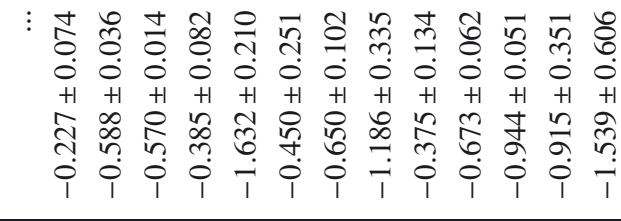 \\
\hline 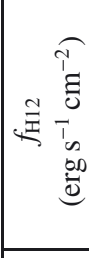 & 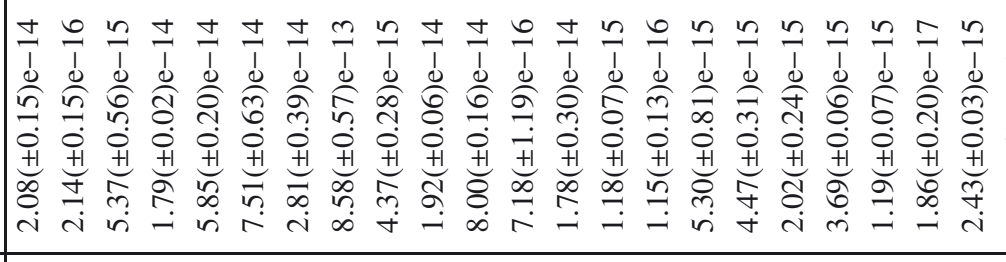 & 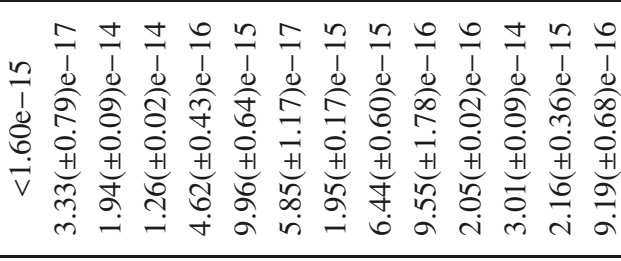 \\
\hline 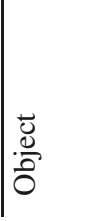 & 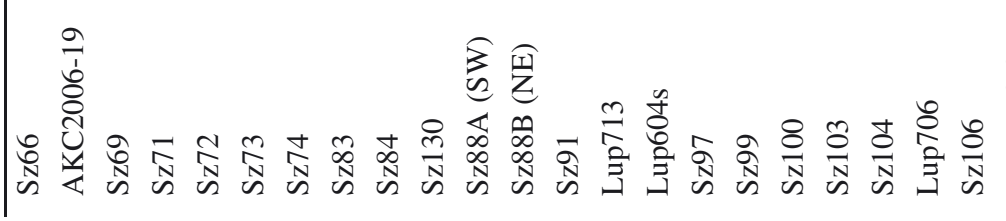 & 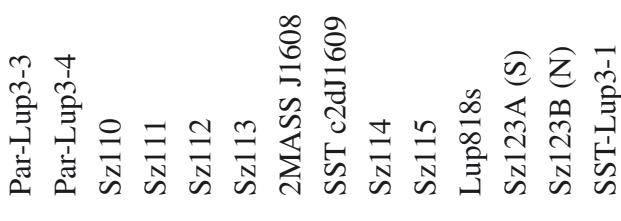 \\
\hline
\end{tabular}




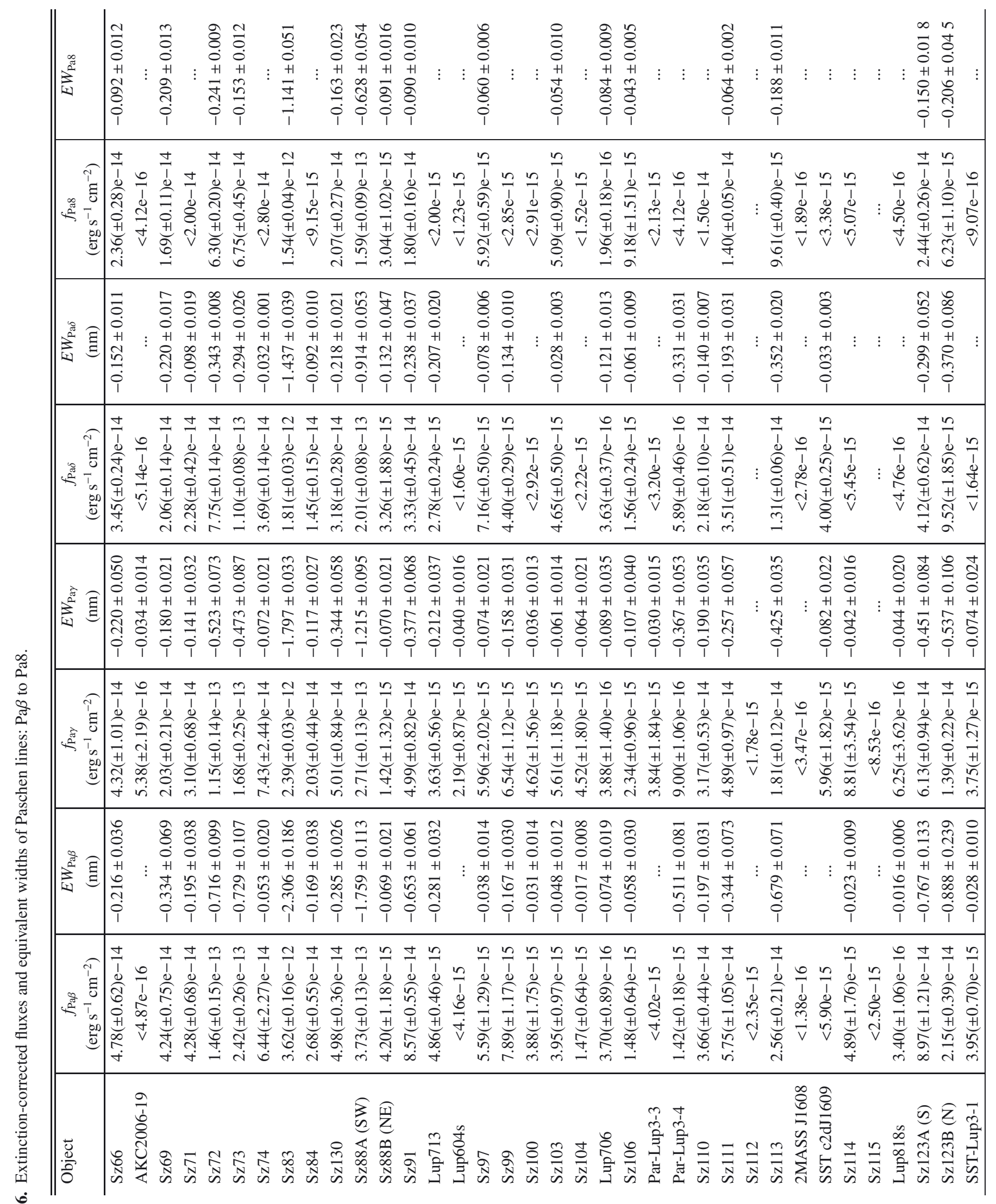




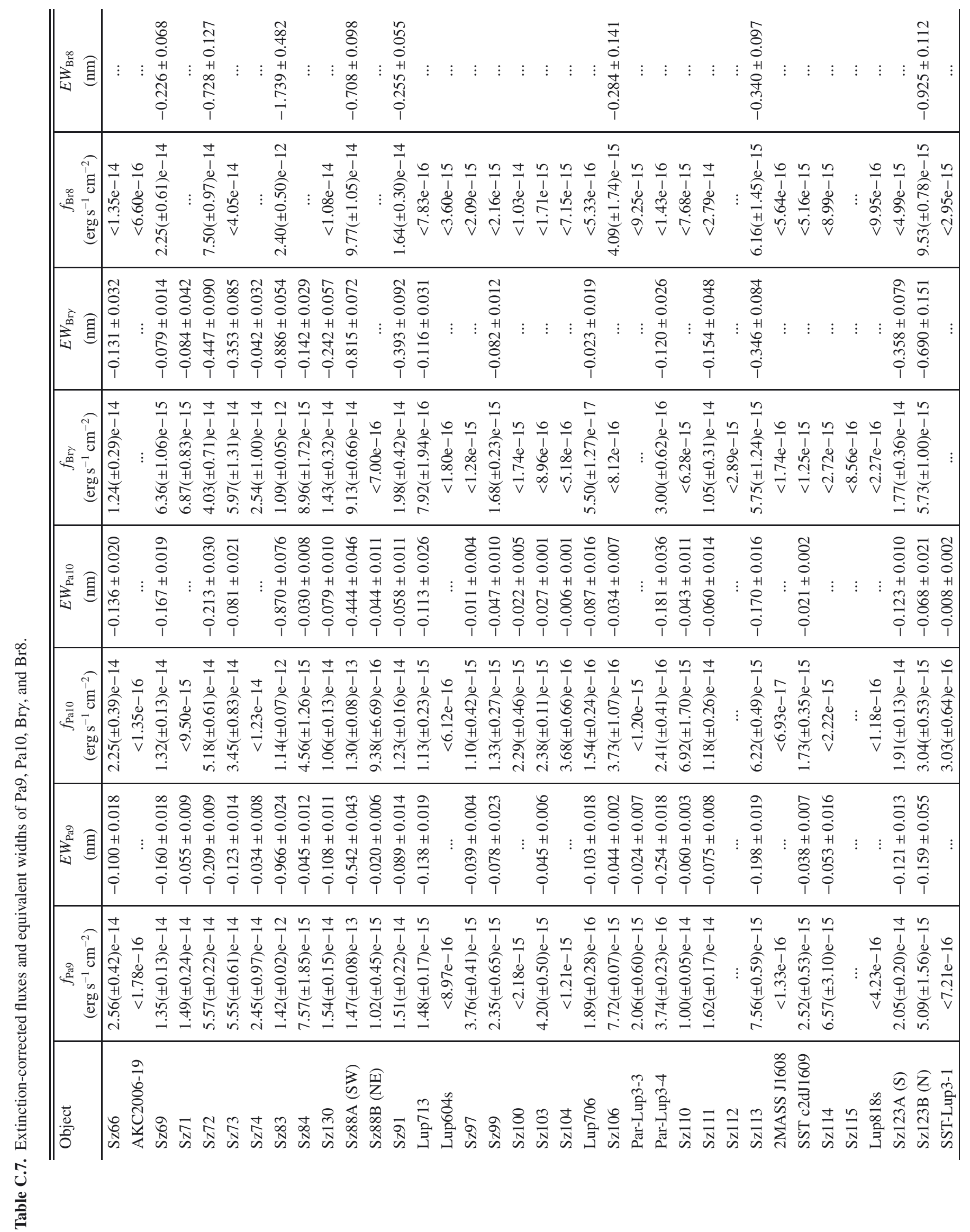




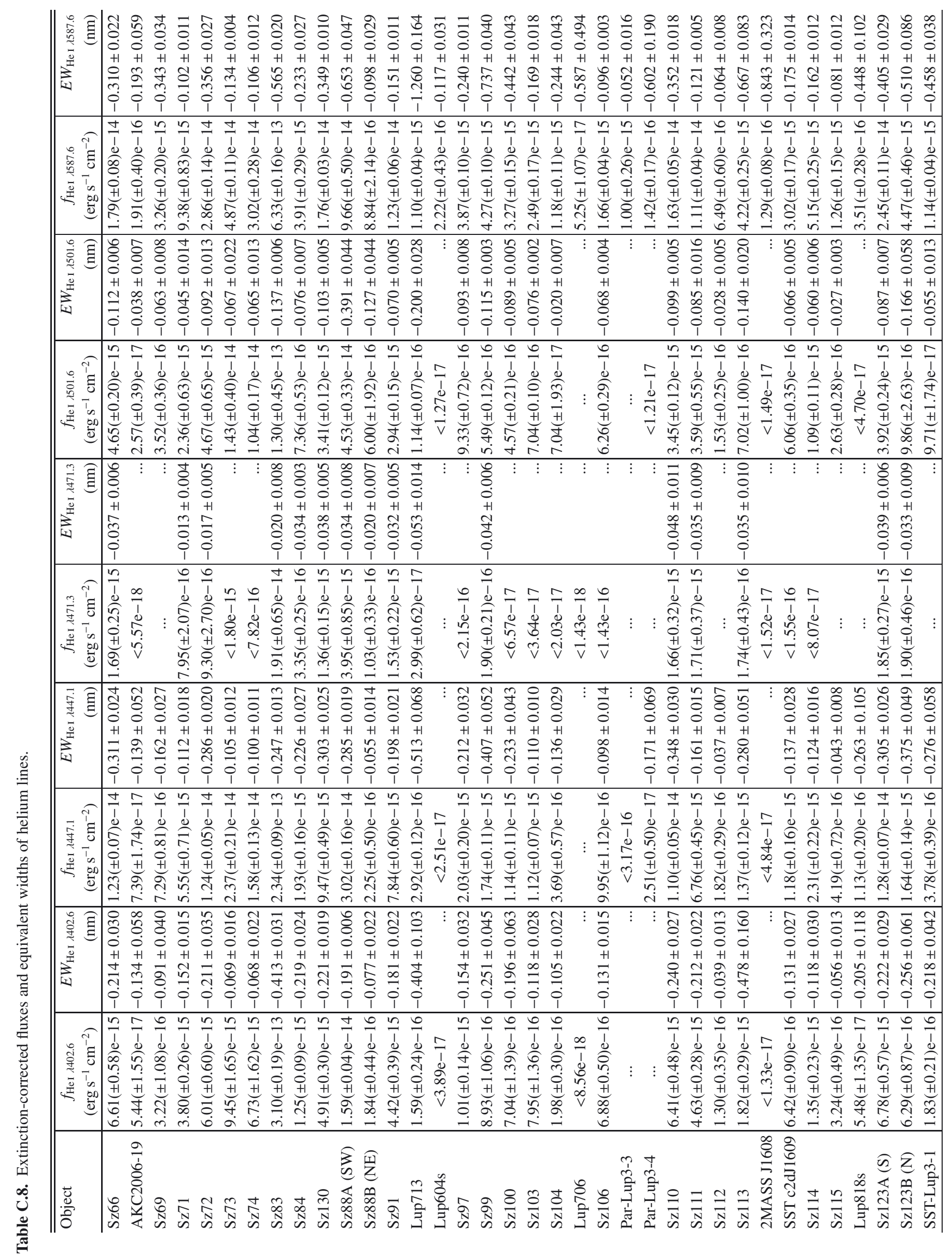




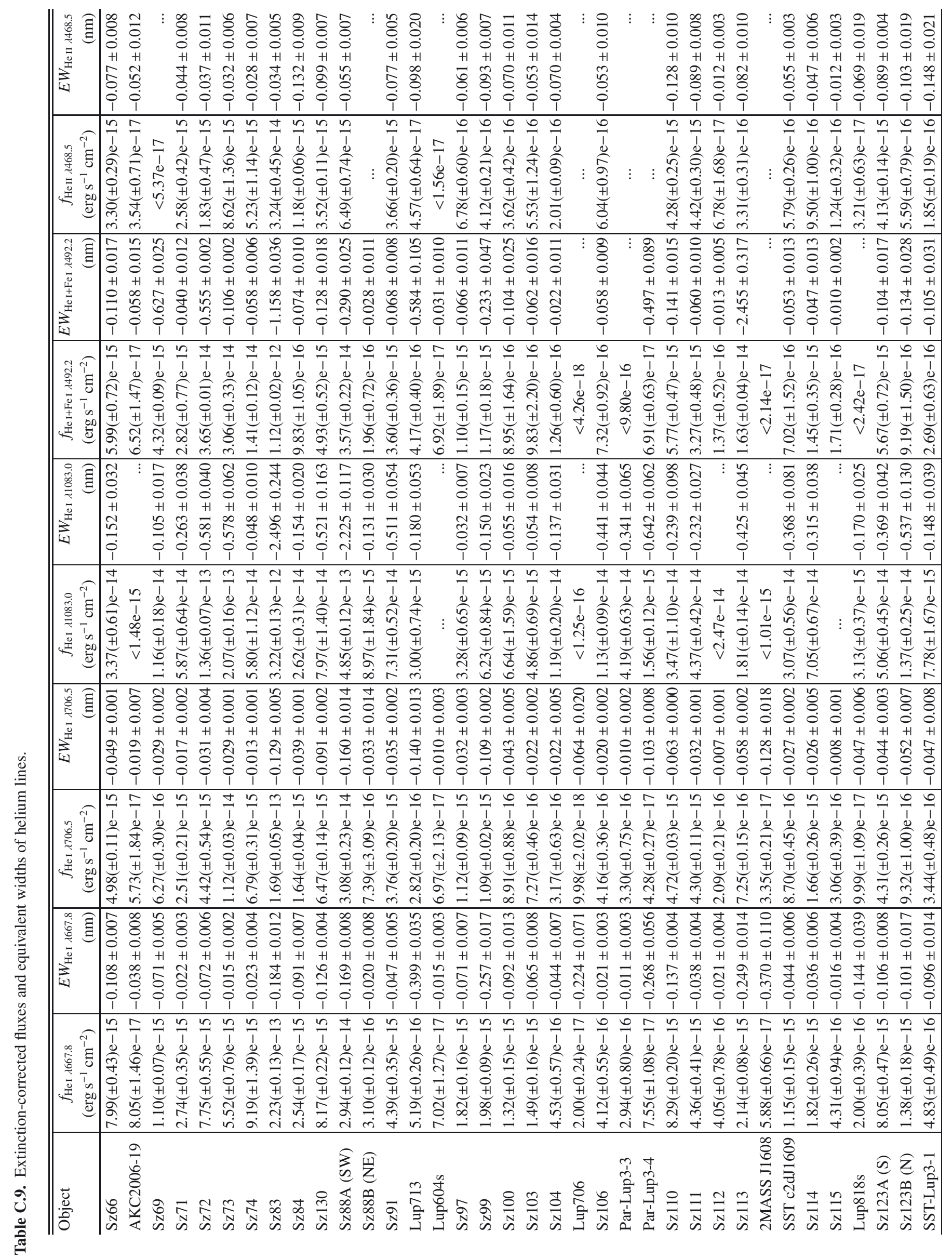




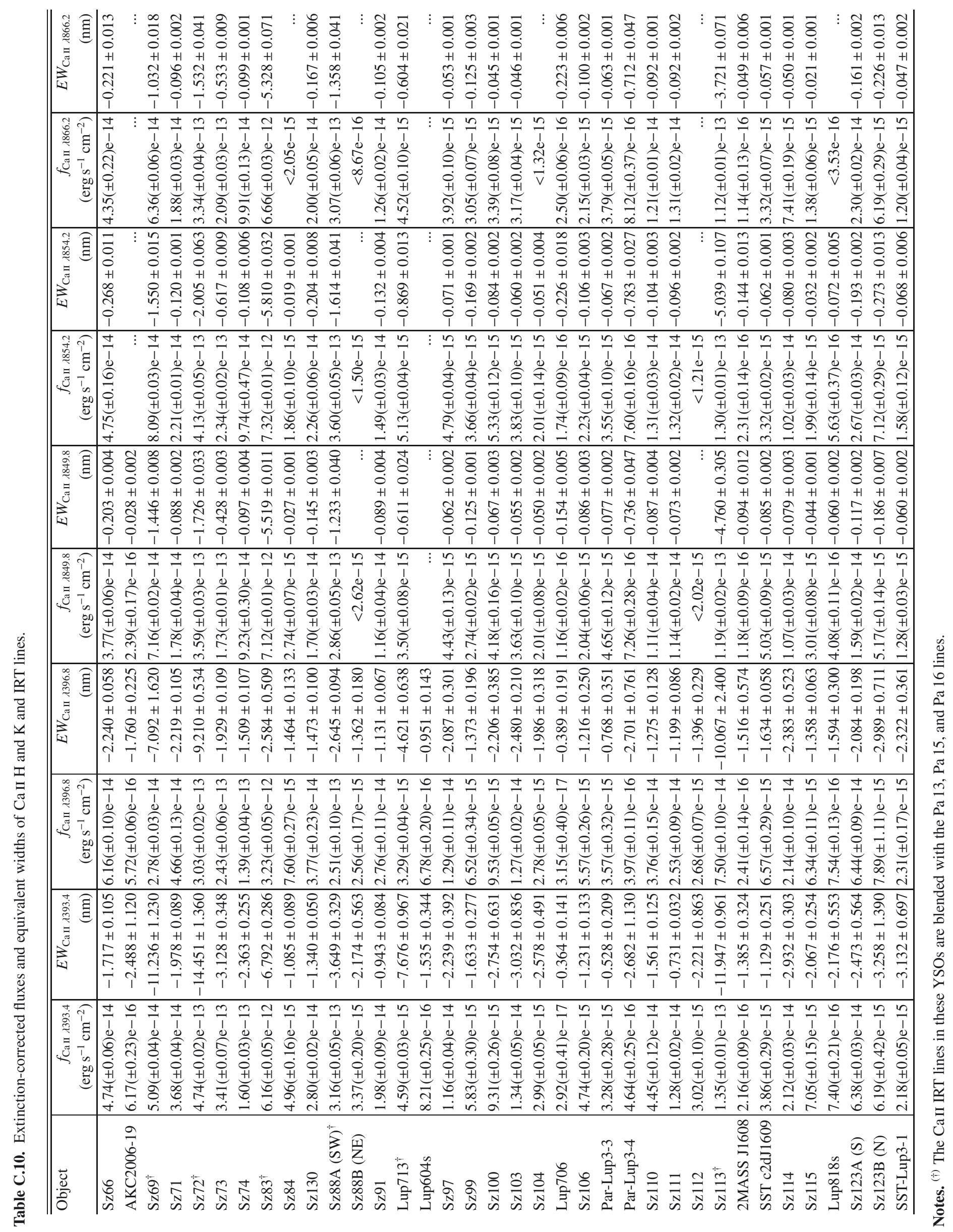

A2, page 36 of 43 
J. M. Alcalá et al.: Accretion in Lupus YSOs

Table C.11. Extinction-corrected fluxes and equivalent widths of the Na I D lines.

\begin{tabular}{|c|c|c|c|c|}
\hline Object & $\begin{array}{c}f_{\mathrm{NaI}} \lambda 588.99 \\
\left(\mathrm{erg} \mathrm{s}^{-1} \mathrm{~cm}^{-2}\right)\end{array}$ & $\begin{array}{r}E W_{\text {NaI } 1} \lambda 588.99 \\
(\mathrm{~nm})\end{array}$ & $\begin{array}{c}f_{\mathrm{NaI}} \lambda 589.59 \\
\left(\mathrm{erg} \mathrm{s}^{-1} \mathrm{~cm}^{-2}\right)\end{array}$ & $\begin{array}{r}E W_{\text {Na I }} \lambda 589.59 \\
(\mathrm{~nm})\end{array}$ \\
\hline Sz66 & $2.03( \pm 0.04) \mathrm{e}-14$ & $-0.335 \pm 0.045$ & $1.43( \pm 0.06) \mathrm{e}-14$ & $-0.236 \pm 0.043$ \\
\hline AKC2006-19 & $5.73( \pm 1.72) \mathrm{e}-17$ & $-0.049 \pm 0.026$ & $7.36( \pm 1.04) \mathrm{e}-17$ & $-0.063 \pm 0.032$ \\
\hline Sz69 & $7.20( \pm 0.34) \mathrm{e}-15$ & $-0.709 \pm 0.049$ & $5.17( \pm 0.29) \mathrm{e}-15$ & $-0.509 \pm 0.047$ \\
\hline Sz71 & $1.57( \pm 0.02) \mathrm{e}-14$ & $-0.164 \pm 0.025$ & $1.23( \pm 0.02) \mathrm{e}-14$ & $-0.128 \pm 0.022$ \\
\hline Sz72 & $5.37( \pm 0.17) \mathrm{e}-14$ & $-0.652 \pm 0.041$ & $3.84( \pm 0.17) \mathrm{e}-14$ & $-0.466 \pm 0.032$ \\
\hline Sz73 & $7.00( \pm 0.05) \mathrm{e}-14$ & $-0.186 \pm 0.021$ & $4.99( \pm 0.09) \mathrm{e}-14$ & $-0.132 \pm 0.017$ \\
\hline Sz74 & $2.79( \pm 0.14) \mathrm{e}-14$ & $-0.090 \pm 0.023$ & $1.53( \pm 0.14) \mathrm{e}-14$ & $-0.049 \pm 0.016$ \\
\hline Sz83 & $7.82( \pm 0.12) \mathrm{e}-13$ & $-0.658 \pm 0.025$ & $6.12( \pm 0.15) \mathrm{e}-13$ & $-0.516 \pm 0.024$ \\
\hline Sz84 & $4.53( \pm 0.98) \mathrm{e}-16$ & $-0.026 \pm 0.015$ & $2.06( \pm 0.35) \mathrm{e}-16$ & $-0.012 \pm 0.009$ \\
\hline Sz130 & $1.45( \pm 0.07) \mathrm{e}-14$ & $-0.279 \pm 0.034$ & $9.28( \pm 0.51) \mathrm{e}-15$ & $-0.178 \pm 0.022$ \\
\hline Sz88A (SW) & $9.31( \pm 0.24) \mathrm{e}-14$ & $-0.584 \pm 0.031$ & $6.59( \pm 0.19) \mathrm{e}-14$ & $-0.414 \pm 0.022$ \\
\hline Sz88B (NE) & $1.70( \pm 0.04) \mathrm{e}-15$ & $-0.161 \pm 0.041$ & $1.01( \pm 0.07) \mathrm{e}-15$ & $-0.100 \pm 0.029$ \\
\hline Sz91 & $1.25( \pm 0.01) \mathrm{e}-14$ & $-0.158 \pm 0.034$ & $8.63( \pm 0.60) e-15$ & $-0.109 \pm 0.025$ \\
\hline Lup713 & $1.29( \pm 0.07) \mathrm{e}-15$ & $-1.280 \pm 0.060$ & $8.07( \pm 0.83) \mathrm{e}-16$ & $-0.803 \pm 0.058$ \\
\hline Lup604s & $7.44( \pm 1.10) \mathrm{e}-17$ & $-0.030 \pm 0.014$ & $3.90( \pm 0.62) \mathrm{e}-17$ & $-0.016 \pm 0.019$ \\
\hline Sz97 & $1.48( \pm 0.07) \mathrm{e}-15$ & $-0.086 \pm 0.018$ & $7.17( \pm 0.51) \mathrm{e}-16$ & $-0.042 \pm 0.012$ \\
\hline Sz99 & $2.29( \pm 0.27) \mathrm{e}-15$ & $-0.374 \pm 0.034$ & $1.47( \pm 0.17) \mathrm{e}-15$ & $-0.240 \pm 0.027$ \\
\hline Sz100 & $7.76( \pm 0.65) \mathrm{e}-16$ & $-0.092 \pm 0.020$ & $5.06( \pm 0.44) \mathrm{e}-16$ & $-0.060 \pm 0.018$ \\
\hline Sz103 & $4.89( \pm 1.03) \mathrm{e}-16$ & $-0.031 \pm 0.012$ & $2.59( \pm 1.25) \mathrm{e}-16$ & $-0.016 \pm 0.010$ \\
\hline Sz104 & $6.53( \pm 0.26) \mathrm{e}-16$ & $-0.107 \pm 0.014$ & $3.79( \pm 0.67) \mathrm{e}-16$ & $-0.062 \pm 0.014$ \\
\hline Lup706 & $1.12( \pm 0.99) \mathrm{e}-17$ & $-0.084 \pm 0.092$ & $9.30( \pm 4.33) \mathrm{e}-18$ & $-0.070 \pm 0.072$ \\
\hline Sz106 & $2.84( \pm 0.09) \mathrm{e}-15$ & $-0.161 \pm 0.026$ & $1.96( \pm 0.05) \mathrm{e}-15$ & $-0.111 \pm 0.022$ \\
\hline Par-Lup3-3 & $2.15( \pm 0.39) \mathrm{e}-15$ & $-0.102 \pm 0.058$ & $1.58( \pm 0.12) \mathrm{e}-15$ & $-0.075 \pm 0.041$ \\
\hline Par-Lup3-4 & $1.31( \pm 0.20) \mathrm{e}-16$ & $-0.429 \pm 0.111$ & $7.97( \pm 3.13) \mathrm{e}-17$ & $-0.260 \pm 0.111$ \\
\hline Sz110 & $7.47( \pm 0.26) \mathrm{e}-15$ & $-0.157 \pm 0.038$ & $3.93( \pm 0.16) \mathrm{e}-15$ & $-0.083 \pm 0.028$ \\
\hline Sz111 & $6.40( \pm 0.13) \mathrm{e}-15$ & $-0.071 \pm 0.021$ & $4.29( \pm 0.48) \mathrm{e}-15$ & $-0.048 \pm 0.014$ \\
\hline Sz112 & $4.88( \pm 0.52) \mathrm{e}-16$ & $-0.043 \pm 0.015$ & $2.64( \pm 0.20) e-16$ & $-0.023 \pm 0.016$ \\
\hline Sz113 & $1.13( \pm 0.03) \mathrm{e}-14$ & $-1.673 \pm 0.050$ & $8.68( \pm 0.26) \mathrm{e}-15$ & $-1.282 \pm 0.056$ \\
\hline 2MASS J1608 & $3.72( \pm 0.84) \mathrm{e}-17$ & $-0.438 \pm 0.177$ & $2.21( \pm 1.53) \mathrm{e}-17$ & $-0.260 \pm 0.193$ \\
\hline SST c2dJ1609 & $3.22( \pm 0.26) \mathrm{e}-16$ & $-0.017 \pm 0.014$ & $8.86( \pm 3.12) \mathrm{e}-17$ & $-0.005 \pm 0.010$ \\
\hline Sz114 & $2.18( \pm 0.11) \mathrm{e}-15$ & $-0.065 \pm 0.008$ & $1.56( \pm 0.19) \mathrm{e}-15$ & $-0.047 \pm 0.008$ \\
\hline Sz115 & $5.77( \pm 1.71) \mathrm{e}-16$ & $-0.031 \pm 0.008$ & $2.92( \pm 2.15) \mathrm{e}-16$ & $-0.016 \pm 0.008$ \\
\hline Lup818s & $2.78( \pm 0.07) \mathrm{e}-16$ & $-0.259 \pm 0.087$ & $2.19( \pm 0.09) \mathrm{e}-16$ & $-0.205 \pm 0.072$ \\
\hline Sz123A (S) & $2.10( \pm 0.03) \mathrm{e}-14$ & $-0.330 \pm 0.039$ & $1.36( \pm 0.02) \mathrm{e}-14$ & $-0.214 \pm 0.026$ \\
\hline Sz123B (N) & $3.34( \pm 0.17) \mathrm{e}-15$ & $-0.347 \pm 0.043$ & $2.03( \pm 0.11) \mathrm{e}-15$ & $-0.211 \pm 0.027$ \\
\hline SST-Lup3-1 & $2.37( \pm 0.23) \mathrm{e}-16$ & $-0.084 \pm 0.019$ & $1.66( \pm 0.18) \mathrm{e}-16$ & $-0.058 \pm 0.019$ \\
\hline
\end{tabular}



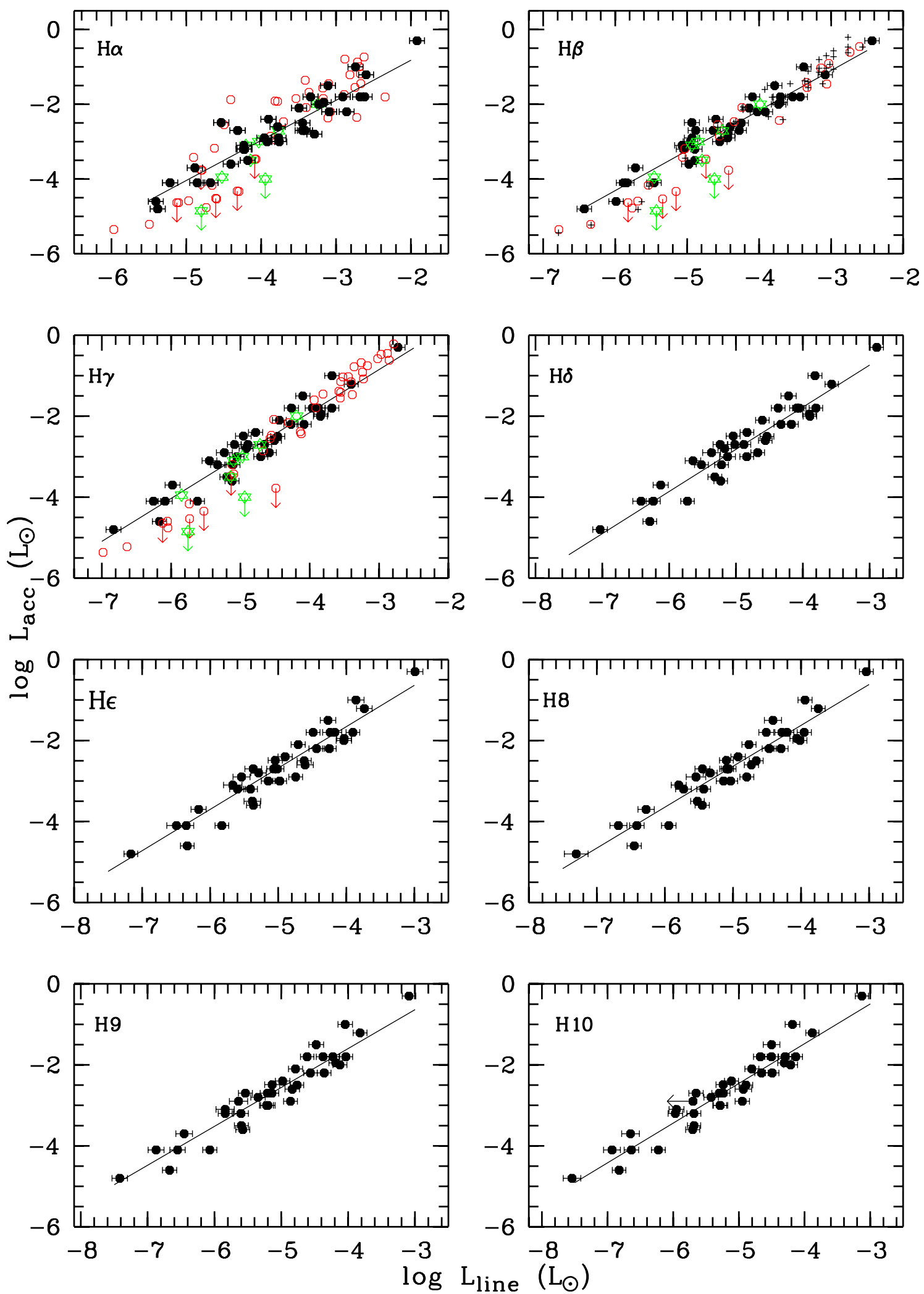

Fig. C.5. Relationships between accretion luminosity and line luminosity for the several diagnostics as labelled in each panel. The Lupus YSOs are represented as black dots. The $\mathrm{H} \alpha, \mathrm{H} \beta$, and $\mathrm{H} \gamma$ data available in literature for YSOs in Taurus (Herczeg \& Hillenbrand 2008) and the $\sigma-O r i$ cluster (Rigliaco et al. 2012) are overlaid as open circles and star symbols, respectively. 
J. M. Alcalá et al.: Accretion in Lupus YSOs
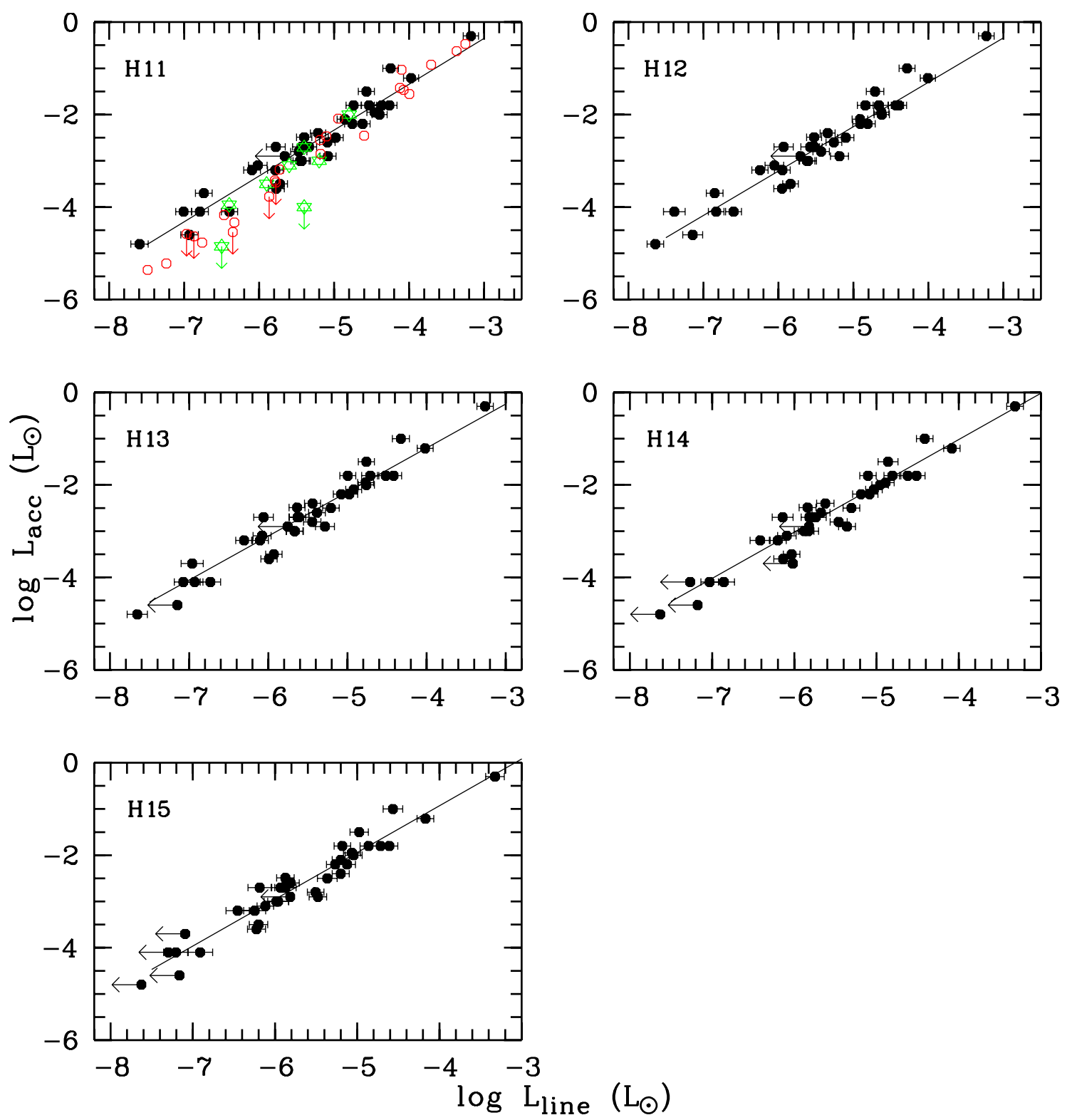

Fig. C.6. Relationships between accretion luminosity and line luminosity for the several diagnostics as labelled in each panel. The Lupus YSOs are represented as black dots. The H11 data available in literature for YSOs in Taurus (Herczeg \& Hillenbrand 2008) and the $\sigma$-Ori cluster (Rigliaco et al. 2012) are overlaid as open circles and star symbols, respectively. 

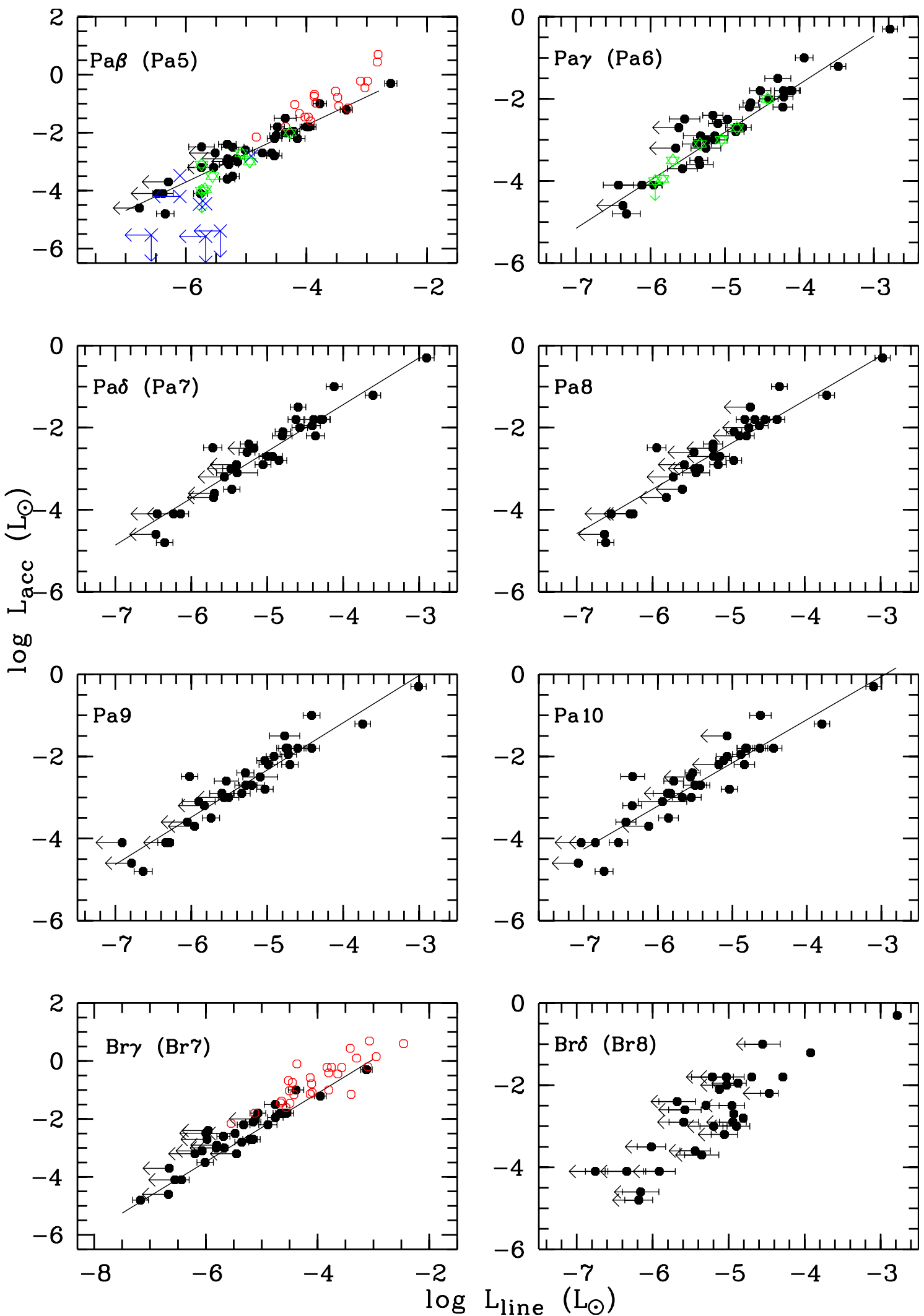

Fig. C.7. Relationships between accretion luminosity and line luminosity for the several diagnostics as labelled in each panel. The Lupus YSOs are represented as black dots. The $\mathrm{Pa} \beta, \mathrm{Pa} \gamma$, and $\mathrm{Br} \gamma$ data available in literature for YSOs in Taurus (Muzerolle et al. 1998; Calvet et al. 2000, 2004), $\rho$-Oph and Chamaeleon (Natta et al. 2004), and the $\sigma$-Ori cluster (Rigliaco et al. 2012) are overlaid as open circles, $\times$-symbols, and star symbols, respectively. 
J. M. Alcalá et al.: Accretion in Lupus YSOs
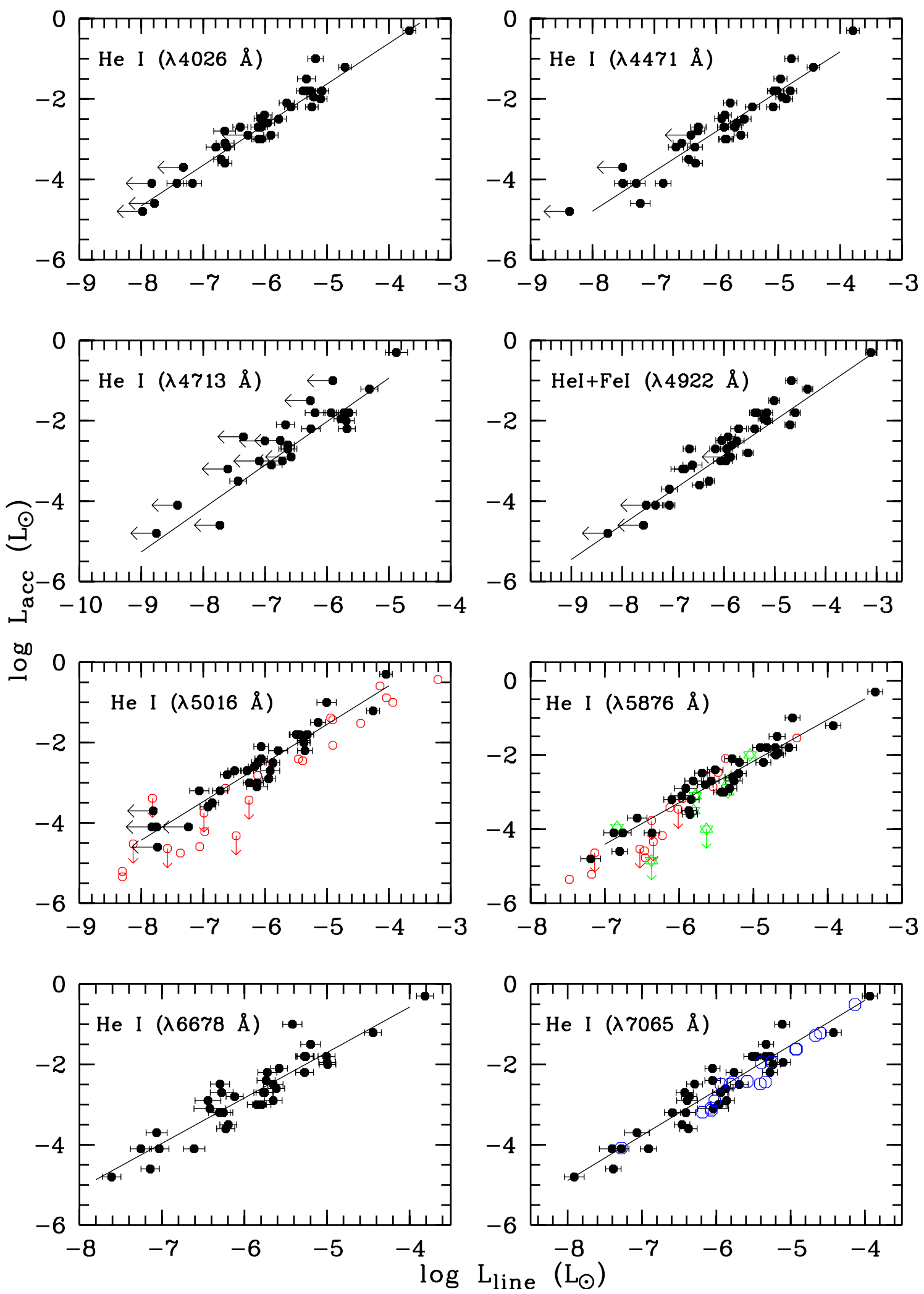

Fig. C.8. Relationships between accretion luminosity and line luminosity for the several diagnostics as labelled in each panel. The Lupus YSOs are represented as black dots. The He I $(\lambda 5016 \AA)$, He I $(\lambda 5876 \AA)$, and He I $(\lambda 7065 \AA)$ data available in literature for YSOs in Taurus $($ Herczeg $\&$ Hillenbrand 2008), the $\sigma$-Ori cluster (Rigliaco et al. 2012), and the Cha-II cloud (Biazzo et al. 2012) are overlaid as small open circles, star symbols, and as open circles, respectively. 

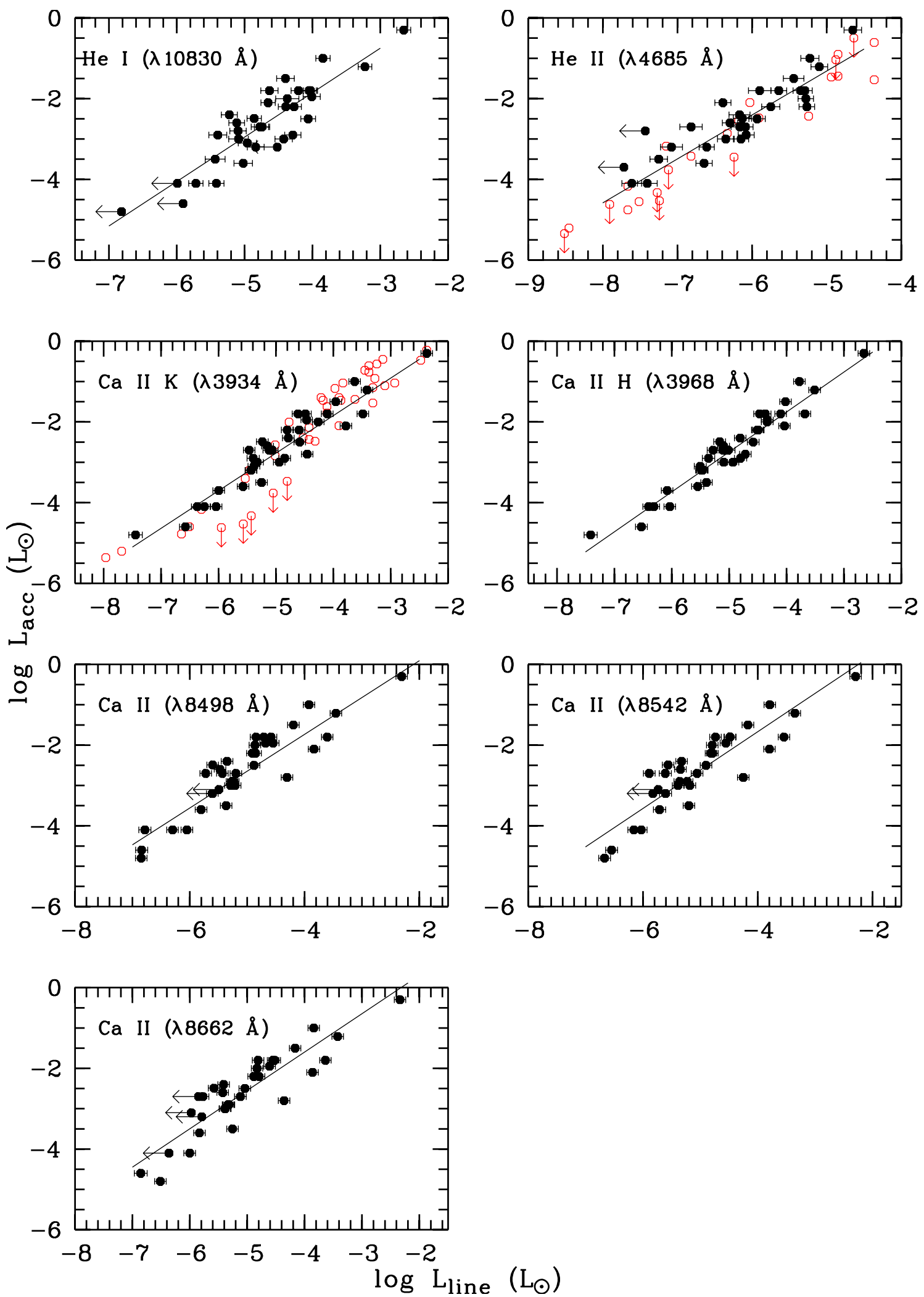

Fig. C.9. Relationships between accretion luminosity and line luminosity for the several diagnostics as labelled in each panel. The Lupus YSOs are represented as black dots. The He II ( $\lambda 4685 \AA$ A) and Ca II K data available in literature for YSOs in Taurus (Herczeg \& Hillenbrand 2008) are overlaid as open circles. 
J. M. Alcalá et al.: Accretion in Lupus YSOs
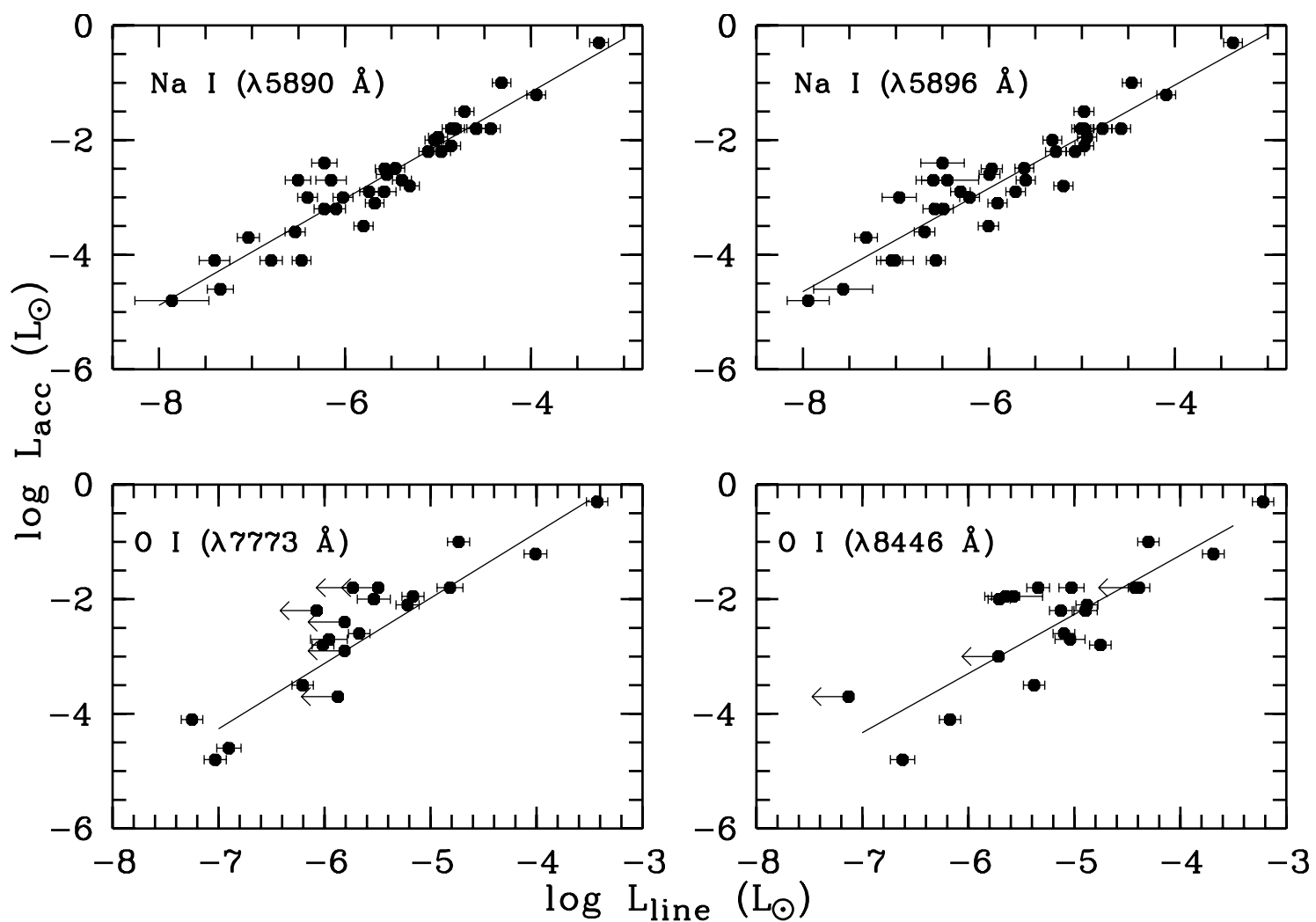

Fig. C.10. Relationships between accretion luminosity and line luminosity for the several diagnostics as labelled in each panel. The Lupus YSOs are represented as black dots. 